\title{
New Antifungal 1,2,4-Triazoles with Difluoro(substituted sulfonyl)methyl Moiety
}

Hiromichi Eto, * Yasushi Kaneko, Sunao Takeda, Minoru Tokizawa, Susumu Sato, Kouiti Yoshida, Setsuo Namiki, Masaki Ogawa, Kazuki Maebashi, Kazuya Ishida, Masaru Matsumoto, and Takemitsu AsAoKA

Central Research Labs., SS Pharmaceutical Co., Ltd., 1143 Nanpeidai, Narita, Chiba 286-8511, Japan.

Received August 24, 2000; accepted November 14, 2000

New 1,2,4-triazoles (2) having a difluoro(substituted sulfonyl)methyl moiety were designed and synthesized via $\alpha, \alpha$-difluoro- $\alpha$-(substituted thio)acetophenones (3). Compounds (2) showed potent antifungal activities against $C$. albicans, $C$. krusei, $A$. flavus and $A$. fumigatus in vitro and against $C$. albicans in vivo for oral and i.v. administrations. Especially, (-)-2a, (-)-2b and (-)-2d showed potent antifungal activities.

Key words antifungal; 1,2,4-triazole; difluoro(substituted sulfonyl)methyl derivative

In our previous paper, ${ }^{1)}$ we reported the preparation of the 1,2,4-triazole derivatives (1) with difluoro(heteroaryl)methyl moiety and their antifungal activities. The introduction of fluorine atoms enhanced their antifungal activities in vitro and some of them showed superior activities compared to those of fluconazole (FLCZ) and itraconazole (ITCZ) against Candida albicans (C. albicans), Candida Krusei (C. Krusei), Trichophyton mentagrophytes (T. mentagrophytes), and Trichophyton rubrum (T. rubrum), and equal to those of ITCZ against Aspergillus fumigatus (A. fumigatus) and Aspergillus flavus (A. flavus). The antifungal azole, which has a sulfonyl or sulfenyl moiety such as Sch424272) and R-102557, ${ }^{3)}$ were developed and showed excellent antifungal activities in vivo. We also reported that SSY $726,{ }^{4)}$ which has a sulfone moiety, showed excellent antifungal activities against Candida species in vivo.

Therefore, we designed new 1,2,4-triazole derivatives (2) with the difluoro(substituted sulfonyl)methyl moiety. In this paper, we describe the preparation of compounds (2) via key intermediates (3) and their antifungal activities.

Chemistry The key intermediates (3) were prepared by three routes. One route is the fluorination of acetophenones (4) by electrophilic fluorinating reagents (route A). The second route is the reaction of ethyl 2,2-difluoro-2-(substituted thio)acetate (6) with phenyllithiums (route B), and the third route is the reaction of $\alpha$-chloro- $\alpha, \alpha$-difluoroacetophenone
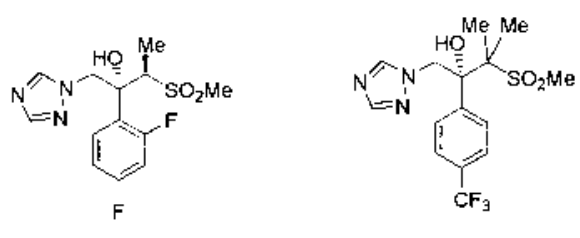

Sch42427 (Schering-Plógh)

\$SY726 (SSP. Welfide)

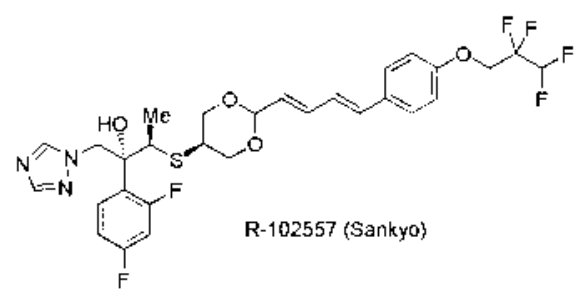

Fig. 1 with mercaptans (route C).

$3 \mathbf{a}-\mathbf{e}$ and $3 \mathbf{g}-\mathbf{I}$ were prepared from the corresponding acetophenones (5) by route A as our previous paper. ${ }^{5)}$ However, the difluorination of $\mathbf{5 n}\left(\mathrm{R}=\mathrm{C}_{6} \mathrm{H}_{5}\right)$ and $50(\mathrm{R}=4$ $\left.\mathrm{CH}_{3}-\mathrm{C}_{6} \mathrm{H}_{4}\right)$ did not proceed completely and a decomposition

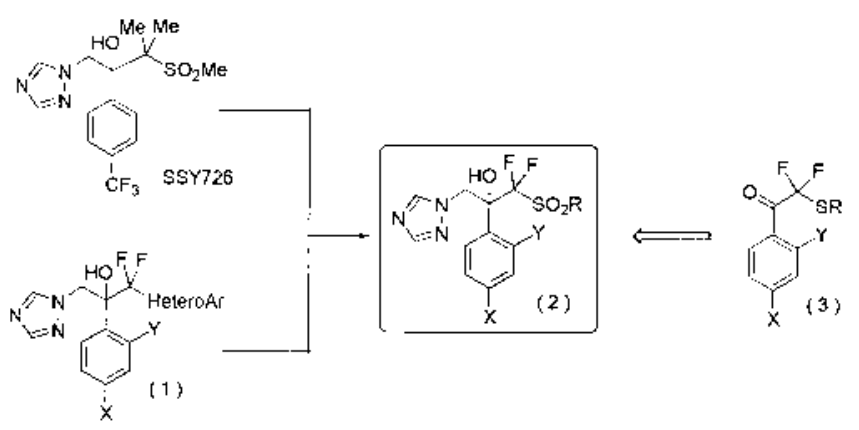

Chart 1

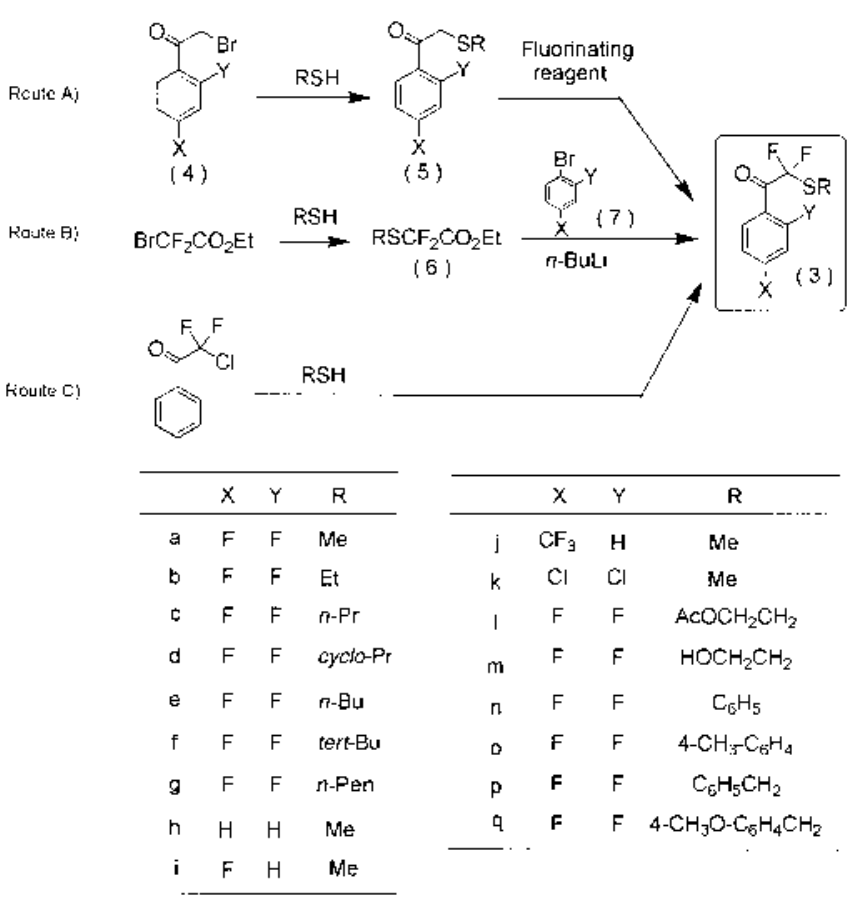

Chart 2 

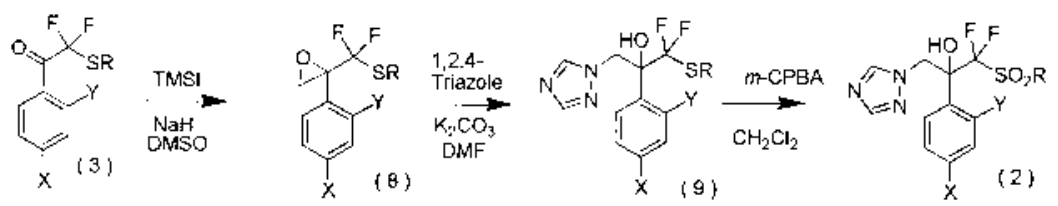

Chart 3
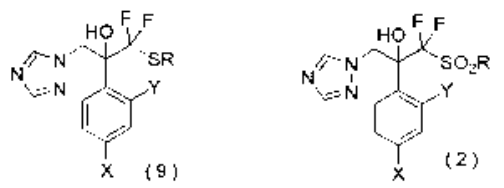

$\mid \begin{aligned} & \mathrm{Rx} \\ & \text { NaH. OMso }\end{aligned}$
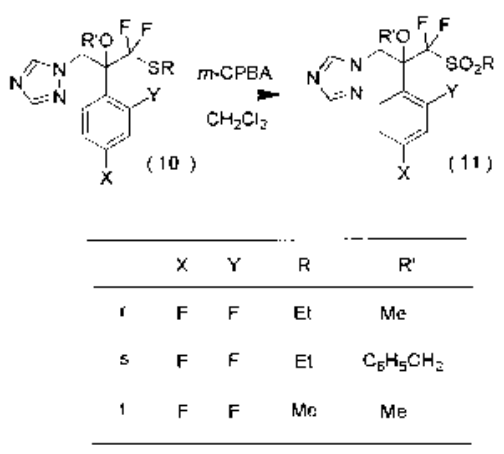

Chart 4

reaction was competed. The fluorination of $\mathbf{5 f}, \mathbf{5 p}$ and $\mathbf{5 q} \mathrm{did}$ not proceed because of their degradation. Therefore, $\mathbf{3 f}$ and 3n-q were prepared from ethyl difluoro(substituted thio)acetate (6) by route B. $\mathbf{3 h}$ was also prepared by route C, but attempt to prepare $\mathbf{3 a}$ from route $\mathrm{C}$ by the methylthiolation of $\alpha$-chloro- $\alpha, \alpha-2^{\prime}, 4^{\prime}$-tetrafluoroacetophenone ${ }^{6)}$ was failed to give a complicated product, because the substitution reaction of the aromatic ring at the 2 and/or 4 position competed.

Compound 2 was prepared from the key intermediates (3) as shown in Chart 3. ${ }^{7 \text { ) }}$

Compound $\mathbf{2 m}$ was prepared from 91 by hydrolysis followed by oxidation.

Compounds 10r and $10 \mathrm{~s}$ were prepared from $9 \mathrm{~b}$ by alkylation. Also compounds 11r and 11s were prepared from 10r and 10s by oxidation, respectively. Compound $\mathbf{2 a}$ was easily methylated by diazomethane to afford 11t (Chart 4), because the acidity of the hydroxy proton is relatively high due to the strong electron withdrawing effect of the neighboring fluorine atoms.

A desfluoro analog of $\mathbf{2 a}(\mathbf{1 2})^{8)}$ was prepared owing to comparing the activities.

Antifungal Activities The synthesized 1,2,4-triazole derivatives containing the difluoro(substituted sulfonyl)methyl moiety $(\mathbf{2} \mathbf{a}-\mathbf{q}, \mathbf{1 1} \mathbf{r}-\mathbf{t})$ and $\mathbf{1 2}$ were screened for their in vitro activities against C. albicans, C. krusei, A. fumigatus and $A$. flavus, and compared with those of FLCZ and ITCZ. The minimum inhibitory concentrations (MICs) are shown in Table 1.

The introduction of a bulky tert-Bu group (2f) or a hydroxy group into the ethyl group (2m) decrease the antifungal activities in vitro. The alkylation of the hydroxy group



Chart 5

Table 1. Antifungal Activities of Compounds 2, 11, 12, FLCZ and ITCZ in Vitro

\begin{tabular}{|c|c|c|c|c|}
\hline \multirow[b]{2}{*}{ Compounds } & \multicolumn{4}{|c|}{$\mathrm{MIC}(\mu \mathrm{g} / \mathrm{ml})$} \\
\hline & $\begin{array}{c}\text { C. albicans } \\
\text { ATCC } 90028\end{array}$ & $\begin{array}{c}\text { C. krusei } \\
\text { ATCC } 6258\end{array}$ & $\begin{array}{l}\text { A. flavus } \\
\text { IFM } 41935\end{array}$ & $\begin{array}{l}\text { A. fumigatus } \\
\text { IFM } 40808\end{array}$ \\
\hline $2 a$ & 0.25 & 8 & 8 & 8 \\
\hline$(-)-2 a$ & 0.125 & 4 & 4 & 4 \\
\hline$(+)-2 a$ & $>64$ & $>64$ & $>64$ & $>64$ \\
\hline $2 b$ & 0.125 & 1 & 4 & 4 \\
\hline$(-)-2 b$ & 0.063 & 0.5 & 2 & 2 \\
\hline$(+)-2 \mathbf{b}$ & $>64$ & $>64$ & $>64$ & $>64$ \\
\hline $2 c$ & 0.063 & 0.5 & 4 & 4 \\
\hline $2 d$ & 0.125 & 2 & 4 & 4 \\
\hline$(-)-2 d$ & 0.063 & 1 & 1 & 2 \\
\hline$(+)-2 d$ & $>64$ & $>64$ & $>64$ & $>64$ \\
\hline $2 e$ & 0.063 & 0.5 & 4 & 8 \\
\hline $2 f$ & 2 & $>8$ & 64 & 32 \\
\hline $2 g$ & 0.031 & 0.5 & 4 & 8 \\
\hline $2 \mathrm{~h}$ & 2 & $>64$ & $>64$ & $>64$ \\
\hline $2 \mathbf{i}$ & 0.5 & 64 & 64 & $>64$ \\
\hline$(-)-2 i$ & 0.25 & 32 & 32 & 64 \\
\hline$(+)-2 \mathbf{i}$ & $>64$ & $>64$ & $>64$ & $>64$ \\
\hline $2 j$ & 0.25 & 32 & $>64$ & $>64$ \\
\hline$(-)-2 \mathbf{j}$ & 0.125 & 16 & $>64$ & $>64$ \\
\hline$(+)-\mathbf{2} \mathbf{j}$ & $>64$ & $>64$ & $>64$ & $>64$ \\
\hline $2 k$ & 0.25 & 2 & 8 & 32 \\
\hline$(-)-2 k$ & 0.125 & 2 & 4 & 16 \\
\hline$(+)-2 \mathbf{k}$ & $>64$ & $>64$ & $>64$ & $>64$ \\
\hline $2 m$ & 1 & 64 & $>64$ & $>64$ \\
\hline $2 n$ & 1 & 16 & $>64$ & 32 \\
\hline 20 & 0.063 & 4 & 8 & 1 \\
\hline $2 p$ & 0.25 & 8 & 4 & 8 \\
\hline $2 q$ & 0.125 & 2 & 4 & 4 \\
\hline $11 \mathrm{r}$ & 0.5 & $>64$ & 64 & $>64$ \\
\hline $11 \mathrm{~s}$ & $>64$ & $>64$ & $>64$ & $>64$ \\
\hline $11 t$ & 4 & $>64$ & $>64$ & $>64$ \\
\hline 12 & 8 & $>64$ & $>64$ & $>64$ \\
\hline FLCZ & 0.25 & 32 & $>64$ & $>64$ \\
\hline ITCZ & 0.031 & 0.25 & 0.25 & 0.25 \\
\hline
\end{tabular}

$(11 \mathbf{r}-\mathbf{t})$ decreased the antifungal activities in vitro.

Compounds 2, 12 and FLCZ, except for $\mathbf{2 m}$, were tested in vivo against $C$. albicans at the 4 times oral administrations after infection in Table 2.

The introduction of fluorine atoms enhanced the antifungal activities in vitro and in vivo (2a, 12).

As phenyl substituents, the 2,4-difluoro (2a) and 2,4dichloro (2k) derivatives showed superior activity to the un- 
Table 2. In Vivo Antifungal Activities of Compounds 2, 12 and FLCZ against $C$. albicans at the 4 Times Oral Administrations after Infection

\begin{tabular}{|c|c|c|c|}
\hline Compounds & $\begin{array}{c}\text { Dose } \\
(\mathrm{mg} / \mathrm{kg})\end{array}$ & $\begin{array}{c}\text { Mean survival } \\
\text { days } \\
\text { (mean } \pm \text { S.D. })\end{array}$ & $\begin{array}{l}\text { No. of survivors } \\
\text { on day } 14 / \\
\text { tested mice }\end{array}$ \\
\hline \multirow[t]{3}{*}{$2 a$} & 5 & $14.0 \pm 0.0 * * *$ & $5 / 5$ \\
\hline & 1.25 & $14.0 \pm 0.0 * * *$ & $5 / 5$ \\
\hline & 0.313 & $12.2 \pm 1.6^{* * *}$ & $2 / 5$ \\
\hline \multirow{3}{*}{$(-)-2 a$} & 5 & $14.0 \pm 0.0^{* * *}$ & $5 / 5$ \\
\hline & 1.25 & $14.0 \pm 0.0 * * *$ & $5 / 5$ \\
\hline & 0.313 & $13.8 \pm 0.4 * * *$ & $4 / 5$ \\
\hline \multirow[t]{3}{*}{$2 b$} & 5 & $14.0 \pm 0.0^{* * *}$ & $5 / 5$ \\
\hline & 1.25 & $14.0 \pm 0.0 * * *$ & $4 / 5$ \\
\hline & 0.313 & $9.6 \pm 2.3 * * *$ & $0 / 5$ \\
\hline \multirow[t]{3}{*}{$(-)-2 b$} & 5 & $14.0 \pm 0.0 * * *$ & $5 / 5$ \\
\hline & 1.25 & $14.0 \pm 0.0 * * *$ & $5 / 5$ \\
\hline & 0.313 & $11.4 \pm 2.4 * * *$ & $0 / 5$ \\
\hline \multirow[t]{3}{*}{$2 c$} & 5 & $14.0 \pm 0.0 * * *$ & $5 / 5$ \\
\hline & 1.25 & $12.6 \pm 1.9 * * *$ & $2 / 5$ \\
\hline & 0.313 & $3.8 \pm 0.8$ & $0 / 5$ \\
\hline \multirow[t]{3}{*}{ 2d } & 5 & $14.0 \pm 0.0^{* * *}$ & $5 / 5$ \\
\hline & 1.25 & $14.0 \pm 0.0 * * *$ & $4 / 5$ \\
\hline & 0.313 & $13.2 \pm 1.8^{* * *}$ & $3 / 5$ \\
\hline \multirow[t]{3}{*}{$(-)-2 d$} & 5 & $14.0 \pm 0.0 * * *$ & $5 / 5$ \\
\hline & 1.25 & $14.0 \pm 0.0 * * *$ & $5 / 5$ \\
\hline & 0.313 & $13.6 \pm 0.5^{* * *}$ & $2 / 5$ \\
\hline \multirow[t]{3}{*}{$2 e$} & 5 & $13.4 \pm 1.3 * * *$ & $4 / 5$ \\
\hline & 1.25 & $10.8 \pm 1.9 * * *$ & $1 / 5$ \\
\hline & 0.313 & $5.6 \pm 1.9$ & $0 / 5$ \\
\hline \multirow[t]{3}{*}{$2 f$} & 5 & $11.4 \pm 2.4^{* * *}$ & $2 / 5$ \\
\hline & 1.25 & $8.8 \pm 3.2 * * *$ & $0 / 5$ \\
\hline & 0.313 & $3.8 \pm 0.8$ & $0 / 5$ \\
\hline \multirow[t]{3}{*}{$2 g$} & 5 & $11.4 \pm 3.2 * *$ & $2 / 5$ \\
\hline & 1.25 & $5.8 \pm 1.3$ & $0 / 5$ \\
\hline & 0.313 & $5.0 \pm 2.5$ & $0 / 5$ \\
\hline \multirow[t]{2}{*}{$2 \mathrm{~h}$} & 5 & $8.8 \pm 0.8 * * *$ & $0 / 5$ \\
\hline & 1.25 & $6.4 \pm 1.3 * *$ & $0 / 5$ \\
\hline \multirow[t]{3}{*}{$2 \mathbf{i}$} & 5 & $13.8 \pm 0.4 * * *$ & $3 / 5$ \\
\hline & 1.25 & $12.4 \pm 1.5^{* * *}$ & $1 / 5$ \\
\hline & 0.313 & $8.4 \pm 1.1 * * *$ & $0 / 5$ \\
\hline \multirow[t]{3}{*}{$2 \mathbf{j}$} & 5 & $13.2 \pm 1.1 * * *$ & $3 / 5$ \\
\hline & 1.25 & $12.0 \pm 1.2 * * *$ & $0 / 5$ \\
\hline & 0.313 & $6.0 \pm 2.4^{*}$ & $0 / 5$ \\
\hline \multirow[t]{3}{*}{$2 k$} & 5 & $14.0 \pm 0.0 * * *$ & $5 / 5$ \\
\hline & 1.25 & $14.0 \pm 0.0 * * *$ & $5 / 5$ \\
\hline & 0.313 & $10.2 \pm 2.4 * * *$ & $1 / 5$ \\
\hline $2 n$ & 5 & $5.0 \pm 1.6$ & $0 / 5$ \\
\hline 20 & 5 & $3.6 \pm 0.5$ & $0 / 5$ \\
\hline $2 p$ & 5 & $4.6 \pm 1.5$ & $0 / 5$ \\
\hline $2 q$ & 5 & $4.8 \pm 1.1$ & $0 / 5$ \\
\hline 12 & 5 & $8.0 \pm 1.9 * *$ & $0 / 5$ \\
\hline \multirow[t]{3}{*}{ FLCZ } & 5 & $13.0 \pm 1.2 * * *$ & $0 / 5$ \\
\hline & 1.25 & $10.6 \pm 2.3 * * *$ & $0 / 5$ \\
\hline & 0.313 & $6.2 \pm 1.8^{* *}$ & $0 / 5$ \\
\hline Control & & $3.4 \pm 0.6$ & $0 / 10$ \\
\hline
\end{tabular}

Significantly different from the control by Kaplan Meire method (Cox mantel test). $* * * ; p<0.001, * * ; p<0.01, * ; p<0.05$.

substituted (2h), 4-fluoro (2i), and 4-trifluoromethyl (2j) derivatives in vitro and in vivo. As substituent for $\mathrm{R}$, the in vitro activities were increased in the order $\mathrm{Me}(\mathbf{2 a})<\operatorname{Et}(\mathbf{2} \mathbf{b})<n$ - $\operatorname{Pr}$ (2c) $<n$-Bu (2e) $<n$-Pen (2g), but the in vivo activities were decreased in order $\mathrm{Me}(\mathbf{2 a}) \geq \operatorname{Et} \quad(\mathbf{2 b})>n-\operatorname{Pr} \quad(\mathbf{2})>n-\mathrm{Bu}$ (2e) $>n$-Pen (2g). We presume that the reason why the antifungal activities decrease according to alkyl chain length in vivo are due to their increase of hydrophobicity which caused a low absorption. Similarly, 4-methylphenyl (2o), benzyl (2p) and 4-methoxybenzyl (2q) derivatives showed excellent anti-

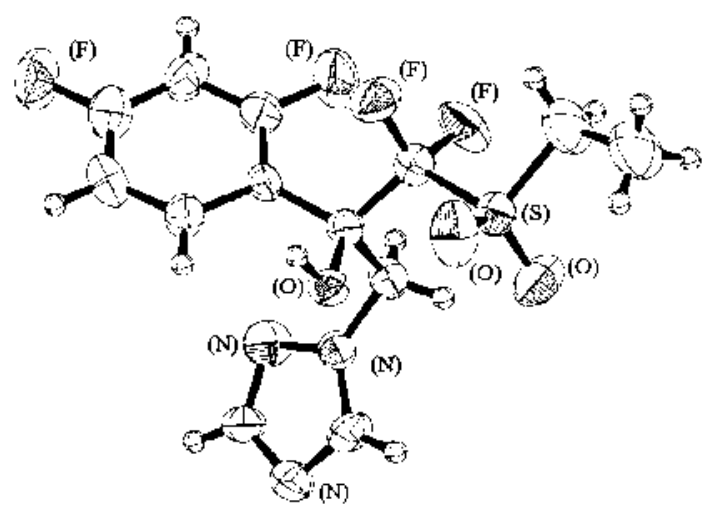

Fig. 2. ORTEP Drawing of (-)-2b (X-Ray Crystallography)

Table 3. In Vivo Antifungal Activities of Compounds (-)-2 and FLCZ against $C$. albicans at a Single Oral Administration after Infection

\begin{tabular}{clcc}
\hline \hline Compounds & $\begin{array}{c}\text { Dose } \\
(\mathrm{mg} / \mathrm{kg})\end{array}$ & $\begin{array}{c}\text { Mean survival } \\
\text { days } \\
\text { (mean } \pm \text { S.D.) }\end{array}$ & $\begin{array}{c}\text { No. of survivors } \\
\text { on day 18/ } \\
\text { tested mice }\end{array}$ \\
\hline$(-)-\mathbf{2 a}$ & 5 & $17.7 \pm 0.9^{* * *}$ & $9 / 10$ \\
& 1.25 & $16.6 \pm 1.3^{* * *}$ & $6 / 10$ \\
$(-)-\mathbf{2} \mathbf{i}$ & 0.313 & $10.6 \pm 2.4^{* * *}$ & $0 / 10$ \\
& 5 & $12.4 \pm 1.8^{* * *}$ & $0 / 10$ \\
& 1.25 & $10.4 \pm 1.6^{* * *}$ & $0 / 10$ \\
$(-)-\mathbf{2} \mathbf{j}$ & 0.313 & $6.0 \pm 1.8^{* * *}$ & $0 / 10$ \\
& 5 & $13.7 \pm 2.1^{* * *}$ & $1 / 10$ \\
$(-)-2 \mathbf{k}$ & 1.25 & $12.4 \pm 1.5^{* * *}$ & $0 / 10$ \\
& 0.313 & $6.9 \pm 1.4^{* * *}$ & $0 / 10$ \\
& 5 & $18.0 \pm 0.0^{* * *}$ & $9 / 10$ \\
FLCZ & 1.25 & $12.6 \pm 1.5^{* * *}$ & $0 / 10$ \\
& 0.313 & $8.3 \pm 1.2^{* * *}$ & $0 / 10$ \\
& 5 & $8.6 \pm 1.4^{* * *}$ & $0 / 5$ \\
Control & 1.25 & $8.1 \pm 1.9^{* * *}$ & $0 / 5$ \\
& 0.313 & $4.8 \pm 1.8^{*}$ & $0 / 5$ \\
& & $3.2 \pm 0.9$ & $0 / 15$ \\
\hline
\end{tabular}

Significantly different from the control by Kaplan Meire method (Cox mantel test). $* * * ; p<0.001, * * ; p<0.01, * ; p<0.05$.

fungal activities in vitro but showed very week antifungal activities in vivo. We postulate that the low in vivo activities of $\mathbf{2 o}, \mathbf{2 p}$ and $\mathbf{2 q}$ are also due to their hydrophobicity. $c$ - $\operatorname{Pr}(\mathbf{2 d})$ derivative showed excellent antifungal activities in vitro and in vivo.

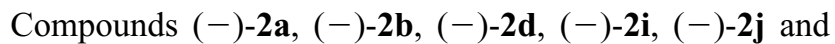
$(-)$-2k showed antifungal activities, but those of the $(+)-$ form showed no significant activities. The absolute structure of $(-)-\mathbf{2 b}$ is $(R)$ as shown in Fig. 2.

Half lives of Sch42427 and SSY726 having methylsufonyl group were relatively long 13 and $44 \mathrm{~h}$ in rats, respectively. Therefore, the (-)-form of $\mathbf{2 a}, \mathbf{2} \mathbf{i}, \mathbf{2} \mathbf{j}$ and $\mathbf{2} \mathbf{k}$, which have methylsulfonyl group, were tested in vivo against $C$. albicans for a single oral administration after infection (Table 3). (-)2a and (-)-2k showed excellent activity at a single oral administration, and (-)-2i and (-)-2j showed superior activities to FLCZ. This suggest that half lives of (-)-2a and (-)$2 \mathbf{k}$ are long and they can be used in a tablet once a day or several days.

The (-)-form of $\mathbf{2 a}, \mathbf{2} \mathbf{b}, \mathbf{2 d}, \mathbf{2 i}$ and $\mathbf{2} \mathbf{k}$ were tested in vivo against $C$. albicans at the 4 times i.v. administrations after infection (Table 4). Their activities were excellent and superior 
Table 4. In Vivo Antifungal Activities of Compounds (-)-2 and FLCZ against $C$. albicans at the 4 Times i.v. Administrations after Infection

\begin{tabular}{clcc}
\hline \hline Compounds & $\begin{array}{c}\text { Dose } \\
(\mathrm{mg} / \mathrm{kg})\end{array}$ & $\begin{array}{c}\text { Mean survival } \\
\text { days } \\
(\text { mean } \pm \text { S.D. })\end{array}$ & $\begin{array}{c}\text { No. of survivors } \\
\text { on day 14/ } \\
\text { tested mice }\end{array}$ \\
\hline$(-)-2 \mathbf{a}$ & 5 & $14.0 \pm 0.0^{* * *}$ & $5 / 5$ \\
& 1.25 & $14.0 \pm 0.0^{* * *}$ & $5 / 5$ \\
$(-)-2 \mathbf{b}$ & 0.313 & $14.0 \pm 0.0^{* * *}$ & $5 / 5$ \\
& 5 & $14.0 \pm 0.0^{* * *}$ & $5 / 5$ \\
& 1.25 & $14.0 \pm 0.0^{* * *}$ & $5 / 5$ \\
$(-)-2 \mathbf{d}$ & 0.313 & $12.6 \pm 1.9^{* * *}$ & $3 / 5$ \\
& 5 & $14.0 \pm 0.0^{* * *}$ & $5 / 5$ \\
$(-)-2 \mathbf{i}$ & 1.25 & $14.0 \pm 0.0^{* * *}$ & $5 / 5$ \\
& 0.313 & $14.0 \pm 0.0^{* * *}$ & $5 / 5$ \\
$(-)-2 \mathbf{k}$ & 5 & $13.6 \pm 0.9^{* * *}$ & $2 / 5$ \\
& 1.25 & $11.8 \pm 1.1^{* * *}$ & $0 / 5$ \\
& 0.313 & $10.6 \pm 1.6^{* * *}$ & $0 / 5$ \\
FLCZ & 5 & $14.0 \pm 0.0^{* * *}$ & $5 / 5$ \\
& 1.25 & $14.0 \pm 0.0^{* * *}$ & $5 / 5$ \\
& 0.313 & $12.2 \pm 1.8^{* * *}$ & $1 / 5$ \\
Control & 5 & $11.6 \pm 1.1^{* * *}$ & $0 / 5$ \\
& 1.25 & $9.6 \pm 1.1^{* * *}$ & $0 / 5$ \\
& 0.313 & $6.0 \pm 1.6^{* *}$ & $0 / 5$ \\
& & $3.5 \pm 0.7$ & $0 / 10$ \\
\hline
\end{tabular}

Significantly different from the control by Kaplan Meire method (Cox mantel test). $* * * ; p<0.001, * * ; p<0.01, * ; p<0.05$

to that of FLCZ. The water solubilities of (-)-2a and (-)-2b are 4.1 and $0.46 \mathrm{mg} / \mathrm{ml}$, respectively. This result suggests that $(-)-\mathbf{2 a}$ and $(-)-\mathbf{2 b}$ can be used in a tablet and/or by injection.

\section{Conclusion}

New 1,2,4-triazoles (2) having a difluoro(substituted sulfonyl)methyl moiety were designed and synthesized via key intermediates (3). The introduction of fluorine atoms into the triazole antifungals enhanced their antifungal activities in vitro and in vivo. The (-)-form of $\mathbf{2}$ is the active form and the absolute configuration of $(-)-\mathbf{2 b}$ is $(R)$. Especially, (-)$\mathbf{2 a},(-)-\mathbf{2 b}$ and $(-)-\mathbf{2 d}$ showed potent activity against $C$. albicans in vivo for both oral and i.v. administrations. They are now undergoing further investigation.

\section{Experimental}

Melting points were determined on a Yanagimoto melting point apparatus without correction. IR spectra were measured on a Nihon-bunko IR-810 spectrometer. ${ }^{1} \mathrm{H}-\mathrm{NMR}$ spectra were recorded on a JOEL JNM-EX400 FTNMR spectrometer in $\mathrm{CDCl}_{3}$ using tetramethylsilane as a respective internal standard. ${ }^{19}$ F-NMR spectra were recorded on a JOEL JNM-EX400 FT-NMR spectrometer in $\mathrm{CDCl}_{3}$ with trifluoroacetic acid as an external standard. The following abbreviations are used: $\mathrm{s}=$ singlet, $\mathrm{d}=$ doublet, $\mathrm{dd}=$ doublet doublet, $\mathrm{dt}=$ doublet triplet, $\mathrm{ddd}=$ doublet doublet doublet, $\mathrm{t}=$ triplet, $\mathrm{q}=$ quartet, $\mathrm{m}=$ multiplet, $\mathrm{br}=$ broad. MS or high-resolution mass spectra (HRMS) were obtained on a JEOL JMS-DX303 or JEOL JMS-AX500 mass spectrometer. Optical rotations were determined on a Horiba SEPA-300 polarimeter. HPLC were performed on a Hitachi L-6000 pump equipped with a Hitachi L-4000 detector. Preparative HPLC were carried out on a Yamazen 800E pump equipped with an UV-10V detector. Column chromatography was carried out on silica gel (BW-80S Fuji-sirial). Resolution of $( \pm)-\mathbf{1}$ was carried out by preparative HPLC on CHIRALCEL OD $(2 \mathrm{~cm}$ i.d. $\times 25 \mathrm{~cm})$ with precolumn CHIRALCEL OD $(2 \mathrm{~cm}$ i.d. $\times 5 \mathrm{~cm})$ (Daicell Chemical Industries, Ltd.) and optical yields were measured by HPLC using CHIRALCEL OD $(4.6 \mathrm{~mm}$ i.d. $\times 250 \mathrm{~mm})$ with pre-column CHIRALCEL OD $(4.6 \mathrm{~mm}$ i.d. $\times$ $50 \mathrm{~mm}$ ) (Daicell Chemical Industries, Ltd.).

General Procedure of Synthesis of 2-(Substituted thio)acetophenone (5) Mercaptans $(50 \mathrm{mmol})$ was added to a solution of 2-haloacetophenone (4) $(55 \mathrm{mmol})$ in dimethyl sulfoxide (DMSO) $(150 \mathrm{ml})$ at room temperature with stirring. The mixture was stirred at room temperature for $15 \mathrm{~h}$. After addition of water, the mixture was extracted with $\mathrm{Et}_{2} \mathrm{O}$. The organic extract was washed with water and brine, dried over $\mathrm{MgSO}_{4}$, and evaporated under reduced pressure. The residue was chromatographed on a silica gel column using AcOEt- $n$-hexane $[1: 9(\mathrm{v} / \mathrm{v})]$ or distilled under reduced pressure to give 5 .

2', 4'-Difluoro-2-(methylthio)acetophenone (5a): Colorless oil. bp $126^{\circ} \mathrm{C} /(14 \mathrm{mmHg})$. Yield $64 \% .{ }^{1} \mathrm{H}-\mathrm{NMR}\left(\mathrm{CDCl}_{3}\right) \delta: 2.08(3 \mathrm{H}, \mathrm{s}), 3.73(2 \mathrm{H}$, d, $\left.J_{\mathrm{HF}}=2.4 \mathrm{~Hz}\right), 6.86-6.91(1 \mathrm{H}, \mathrm{m}), 6.97-7.02(1 \mathrm{H}, \mathrm{m}), 7.98-8.04(1 \mathrm{H}$, m). ${ }^{19} \mathrm{~F}-\mathrm{NMR}\left(\mathrm{CDCl}_{3}\right) \delta:-25.5--25.6(1 \mathrm{~F}, \mathrm{~m}),-27.4-27.5(1 \mathrm{~F}, \mathrm{~m})$. MS $m / z$ (\%): $202\left(14, \mathrm{M}^{+}\right.$), 141 (100). HRMS $m / z: 202.0249$ (Calcd for $\left.\mathrm{C}_{9} \mathrm{H}_{8} \mathrm{~F}_{2} \mathrm{OS}: 202.0264\right)$. IR (neat) $\mathrm{cm}^{-1}: 1675$.

2-(Ethylthio)-2', $4^{\prime}$-Difluoroacetophenone (5b): Colorless oil. bp $143^{\circ} \mathrm{C} /$ $(13 \mathrm{mmHg})$. Yield $89 \% .{ }^{1} \mathrm{H}-\mathrm{NMR}\left(\mathrm{CDCl}_{3}\right) \delta: 1.24(3 \mathrm{H}, \mathrm{t}, J=7.3 \mathrm{~Hz}), 2.52$ $(2 \mathrm{H}, \mathrm{q}, J=7.3 \mathrm{~Hz}), 3.78\left(2 \mathrm{H}, \mathrm{d}, J_{\mathrm{HF}}=2.4 \mathrm{~Hz}\right), 6.86-6.91(1 \mathrm{H}, \mathrm{m}), 6.97-$ $7.01(1 \mathrm{H}, \mathrm{m}), 7.96-8.02(1 \mathrm{H}, \mathrm{m}) .{ }^{19} \mathrm{~F}-\mathrm{NMR}\left(\mathrm{CDCl}_{3}\right) \delta:-25.5-25.6$ (1F, m), $-27.5--27.6(1 \mathrm{~F}, \mathrm{~m})$. MS $m / z(\%): 216\left(2, \mathrm{M}^{+}\right), 141(100)$. HRMS m/z: 216.0427 (Calcd for $\mathrm{C}_{10} \mathrm{H}_{10} \mathrm{~F}_{2} \mathrm{OS}$ : 216.0420). IR (neat) $\mathrm{cm}^{-1}$ : 1675.

$2^{\prime}, 4^{\prime}$-Difluoro-2-(propylthio)acetophenone $(\mathbf{5 c})$ : Colorless oil. bp $112{ }^{\circ} \mathrm{C} /$ $(5 \mathrm{mmHg})$. Yield $85 \%$. ${ }^{1} \mathrm{H}-\mathrm{NMR}\left(\mathrm{CDCl}_{3}\right) \delta: 0.96(3 \mathrm{H}, \mathrm{dt}, J=2.0,7.3 \mathrm{~Hz})$, $1.55-1.65(2 \mathrm{H}, \mathrm{m}), 2.47(2 \mathrm{H}, \mathrm{dt}, J=1.5,7.3 \mathrm{~Hz}), 3.75\left(2 \mathrm{H}, \mathrm{d}, J_{\mathrm{HF}}=2.0 \mathrm{~Hz}\right)$, $6.85-6.90(1 \mathrm{H}, \mathrm{m}), 6.95-7.00(1 \mathrm{H}, \mathrm{m}), 7.95-8.00(1 \mathrm{H}, \mathrm{m}) .{ }^{19} \mathrm{~F}-\mathrm{NMR}$ $\left(\mathrm{CDCl}_{3}\right) \delta:-25.6-25.7(1 \mathrm{~F}, \mathrm{~m}),-27.5-27.6(1 \mathrm{~F}, \mathrm{~m}) . \mathrm{MS} m / z(\%)$ : $230\left(15, \mathrm{M}^{+}\right), 141(100)$. HRMS $m / z$ : 230.0558 (Calcd for $\mathrm{C}_{11} \mathrm{H}_{12} \mathrm{~F}_{2} \mathrm{OS}$ : 230.0577). IR (neat) $\mathrm{cm}^{-1}: 1675$.

2-(Cyclopropylthio)-2', $4^{\prime}$-difluoroacetophenone (5d): Colorless oil. bp $123^{\circ} \mathrm{C} /(3 \mathrm{mmHg})$. Yield $87 \%$. ${ }^{1} \mathrm{H}-\mathrm{NMR}\left(\mathrm{CDCl}_{3}\right) \delta: 0.50-0.54(2 \mathrm{H}, \mathrm{m})$, $0.83-0.88(2 \mathrm{H}, \mathrm{m}), 1.82-1.88(1 \mathrm{H}, \mathrm{m}), 3.86\left(2 \mathrm{H}, \mathrm{d}, J_{\mathrm{HF}}=2.4 \mathrm{~Hz}\right), 6.86-$ $6.91(1 \mathrm{H}, \mathrm{m}), 6.97-7.02(1 \mathrm{H}, \mathrm{m}), 7.97-8.03(1 \mathrm{H}, \mathrm{m}) .{ }^{19} \mathrm{~F}-\mathrm{NMR}\left(\mathrm{CDCl}_{3}\right)$ $\delta:-25.6-25.7(1 \mathrm{~F}, \mathrm{~m}),-27.5-27.6(1 \mathrm{~F}, \mathrm{~m})$. MS $m / z$ (\%): $228(18$, $\mathrm{M}^{+}$), 141 (100). HRMS $m / z$ : 228.0415 (Calcd for $\mathrm{C}_{11} \mathrm{H}_{10} \mathrm{~F}_{2} \mathrm{OS}: 228.0421$ ). IR (neat) $\mathrm{cm}^{-1}: 1680$.

2-(Butylthio)-2', $4^{\prime}$-difluoroacetophenone (5e): Colorless oil. bp $123^{\circ} \mathrm{C} /$ $(5 \mathrm{mmHg})$. Yield $85 \%$. ${ }^{1} \mathrm{H}-\mathrm{NMR}\left(\mathrm{CDCl}_{3}\right) \delta: 0.89(3 \mathrm{H}, \mathrm{t}, J=7.3 \mathrm{~Hz}), 1.35-$ $1.45(2 \mathrm{H}, \mathrm{m}), 1.50-1.60(2 \mathrm{H}, \mathrm{m}), 2.49(2 \mathrm{H}, \mathrm{t}, J=7.3 \mathrm{~Hz}), 3.75(2 \mathrm{H}, \mathrm{d}$, $\left.J_{\mathrm{HF}}=2.0 \mathrm{~Hz}\right), 6.85-6.90(1 \mathrm{H}, \mathrm{m}), 6.95-7.00(1 \mathrm{H}, \mathrm{m}), 7.95-8.00(1 \mathrm{H}, \mathrm{m})$. ${ }^{19} \mathrm{~F}-\mathrm{NMR}\left(\mathrm{CDCl}_{3}\right) \delta:-25.5-25.6(1 \mathrm{~F}, \mathrm{~m}),-27.4-27.5(1 \mathrm{~F}, \mathrm{~m})$. MS $m / z$ (\%): $244\left(21, \mathrm{M}^{+}\right), 141$ (100). HRMS $\mathrm{m} / \mathrm{z}: 244.0746$ (Calcd for $\left.\mathrm{C}_{12} \mathrm{H}_{14} \mathrm{~F}_{2} \mathrm{OS}: 244.0734\right)$. IR (neat) $\mathrm{cm}^{-1}: 1680$.

2-(tert-Butylthio)-2', $4^{\prime}$-difluoroacetophenone (5f): Colorless oil. bp $125^{\circ} \mathrm{C} /(5 \mathrm{mmHg})$. Yield $94 \% .{ }^{1} \mathrm{H}-\mathrm{NMR}\left(\mathrm{CDCl}_{3}\right) \delta: 1.32(9 \mathrm{H}, \mathrm{s}), 3.88(2 \mathrm{H}$, d, $\left.J_{\mathrm{HF}}=2.4 \mathrm{~Hz}\right), 6.86-6.91(1 \mathrm{H}, \mathrm{m}), 6.96-7.01(1 \mathrm{H}, \mathrm{m}), 7.91-7.97(1 \mathrm{H}$, $\mathrm{m}) .{ }^{19} \mathrm{~F}-\mathrm{NMR}\left(\mathrm{CDCl}_{3}\right) \delta:-25.7--25.8(1 \mathrm{~F}, \mathrm{~m}),-27.9-28.0(1 \mathrm{~F}, \mathrm{~m})$. MS $m / z$ (100): 244 (32, $\mathrm{M}^{+}$), 141 (100). HRMS $m / z$ : 244.0752 (Calcd for $\left.\mathrm{C}_{12} \mathrm{H}_{14} \mathrm{~F}_{2} \mathrm{OS}: 244.0734\right)$. IR ( $\left.\mathrm{KBr}\right) \mathrm{cm}^{-1}: 1680$.

$2^{\prime}, 4^{\prime}$-Difluoro-2-(pentylthio)-acetophenone (5g): Colorless oil. bp $137^{\circ} \mathrm{C} /$ $(5 \mathrm{mmHg})$. Yield $81 \%$. ${ }^{1} \mathrm{H}-\mathrm{NMR}\left(\mathrm{CDCl}_{3}\right) \delta: 0.89(3 \mathrm{H}, \mathrm{t}, J=7.3 \mathrm{~Hz}), 1.30-$ $1.80(6 \mathrm{H}, \mathrm{m}), 2.49(2 \mathrm{H}, \mathrm{t}, J=7.3 \mathrm{~Hz}), 3.75\left(2 \mathrm{H}, \mathrm{d}, J_{\mathrm{HF}}=2.4 \mathrm{~Hz}\right), 6.85-6.90$ $(1 \mathrm{H}, \mathrm{m}), 6.95-7.00(1 \mathrm{H}, \mathrm{m}), 7.95-8.00(1 \mathrm{H}, \mathrm{m}) .{ }^{19} \mathrm{~F}-\mathrm{NMR}\left(\mathrm{CDCl}_{3}\right) \delta$ : $-25.6-25.7(1 \mathrm{~F}, \mathrm{~m}),-27.4-27.5(1 \mathrm{~F}, \mathrm{~m})$. MS $m / z(\%): 258\left(23, \mathrm{M}^{+}\right)$, 141 (100). HRMS $m / z$ : 258.3291 (Calcd for $\mathrm{C}_{13} \mathrm{H}_{16} \mathrm{~F}_{2} \mathrm{OS}: 258.3283$ ). IR (neat) $\mathrm{cm}^{-1}: 1680$.

2-(Methylthio)acetophenone (5h): Colorless oil. bp $110^{\circ} \mathrm{C} /(3 \mathrm{mmHg})$. Yield $73 \%$. ${ }^{1} \mathrm{H}-\mathrm{NMR}\left(\mathrm{CDCl}_{3}\right) \delta: 2.14(3 \mathrm{H}, \mathrm{s}), 3.77(2 \mathrm{H}, \mathrm{s}), 7.48(2 \mathrm{H}, \mathrm{t}$, $J=7.3 \mathrm{~Hz}), 7.58(1 \mathrm{H}, \mathrm{dd}, J=1.5,7.3 \mathrm{~Hz}), 7.98(2 \mathrm{H}, \mathrm{dd}, J=1.5,7.3 \mathrm{~Hz}) . \mathrm{MS}$ $\mathrm{m} / \mathrm{z}(\%): 166\left(19, \mathrm{M}^{+}\right), 105(100)$. HRMS m/z: 166.0440 (Calcd for $\mathrm{C}_{9} \mathrm{H}_{10}$ OS: 166.0452). IR (neat) $\mathrm{cm}^{-1}: 1675$.

4'-Fluoro-2-(methylthio)acetophenone (5i): Colorless oil. bp $112^{\circ} \mathrm{C} /$ $(3 \mathrm{mmHg})$. Yield $73 \%$. ${ }^{1} \mathrm{H}-\mathrm{NMR}\left(\mathrm{CDCl}_{3}\right) \delta: 2.14(3 \mathrm{H}, \mathrm{s}), 3.73(2 \mathrm{H}, \mathrm{s})$, $7.10-7.15(2 \mathrm{H}, \mathrm{m}), 8.00-8.05(2 \mathrm{H}, \mathrm{m}) .{ }^{19} \mathrm{~F}-\mathrm{NMR}\left(\mathrm{CDCl}_{3}\right) \delta:-28.9(1 \mathrm{~F}$, s). MS $m / z(\%): 184\left(1, \mathrm{M}^{+}\right), 123(100)$. HRMS $m / z$ : 184.0355 (Calcd for $\left.\mathrm{C}_{9} \mathrm{H}_{9} \mathrm{FOS}: 184.0358\right)$. IR (neat) $\mathrm{cm}^{-1}: 1680$.

2-(Methylthio)-4'-(trifluoromethy)acetophenone (5j): Colorless oil. bp $107^{\circ} \mathrm{C} /(5 \mathrm{mmHg})$. Yield 66\%. ${ }^{1} \mathrm{H}-\mathrm{NMR}\left(\mathrm{CDCl}_{3}\right) \delta: 2.13(3 \mathrm{H}, \mathrm{s}), 3.77(2 \mathrm{H}$, s), $7.75(2 \mathrm{H}, \mathrm{d}, J=7.8 \mathrm{~Hz}), 8.09(2 \mathrm{H}, \mathrm{d}, J=7.8 \mathrm{~Hz}) .{ }^{19} \mathrm{~F}-\mathrm{NMR}\left(\mathrm{CDCl}_{3}\right) \delta$ : $12.6(3 \mathrm{~F}, \mathrm{~s})$. MS $m / z(\%): 234\left(23, \mathrm{M}^{+}\right), 173(100)$. HRMS $m / z: 234.0350$ (Calcd for $\mathrm{C}_{10} \mathrm{H}_{9} \mathrm{~F}_{3} \mathrm{OS}: 234.0326$ ). IR (neat) $\mathrm{cm}^{-1}: 1680$.

$2^{\prime}, 4^{\prime}$-Dichloro-2-(methylthio)acetophenone (5k): Colorless oil. Yield $85 \% .{ }^{1} \mathrm{H}-\mathrm{NMR}\left(\mathrm{CDCl}_{3}\right) \delta: 2.21(3 \mathrm{H}, \mathrm{s}), 3.86(2 \mathrm{H}, \mathrm{s}), 7.43(1 \mathrm{H}, \mathrm{dd}, J=2.0$, $8.3 \mathrm{~Hz}), 7.55(1 \mathrm{H}, \mathrm{d}, J=2.0 \mathrm{~Hz}), 7.63(1 \mathrm{H}, \mathrm{d}, J=8.3 \mathrm{~Hz}) . \mathrm{MS} m / z(\%): 234$ $\left(0.4, \mathrm{M}^{+}\right), 173$ (100). HRMS m/z: 233.9669 (Calcd for $\mathrm{C}_{9} \mathrm{H}_{8} \mathrm{Cl}_{2} \mathrm{OS}$ : 233.9673). IR (neat) $\mathrm{cm}^{-1}: 1690$.

2',4'-Difluoro-2-(2-hydroxyethylthio)acetophenone (5m): Colorless oil. 
Yield $72 \%$. ${ }^{1} \mathrm{H}-\mathrm{NMR}\left(\mathrm{CDCl}_{3}\right) \delta: 2.37(1 \mathrm{H}, \mathrm{br} \mathrm{s}), 2.73(2 \mathrm{H}, \mathrm{t}, J=5.9 \mathrm{~Hz})$, $3.75(2 \mathrm{H}, \mathrm{t}, J=5.9 \mathrm{~Hz}), 3.84\left(2 \mathrm{H}, \mathrm{d}, J_{\mathrm{HF}}=2.4 \mathrm{~Hz}\right), 6.87-6.93(1 \mathrm{H}, \mathrm{m})$, 6.98-7.03 (1H, m), 7.98-8.04 (1H, m). ${ }^{19}$ F-NMR $\left(\mathrm{CDCl}_{3}\right) \delta:-24.7-$ $-24.8(1 \mathrm{~F}, \mathrm{~m}),-27.3--27.4(1 \mathrm{~F}, \mathrm{~m})$. MS $m / z(\%): 214\left(29, \mathrm{M}^{+}-\mathrm{H}_{2} \mathrm{O}\right)$, 141 (100). HRMS $m / z: 214.0258$ (Calcd for $\mathrm{C}_{10} \mathrm{H}_{8} \mathrm{~F}_{2} \mathrm{OS}: 214.0264$ ). IR (neat) $\mathrm{cm}^{-1}: 3425,1675$.

2',4'-Difluoro-2-(phenylthio)acetophenone (5n): Colorless oil. Yield 81\% ${ }^{1} \mathrm{H}-\mathrm{NMR}\left(\mathrm{CDCl}_{3}\right) \delta: 4.15(2 \mathrm{H}, \mathrm{s}), 6.85-6.90(1 \mathrm{H}, \mathrm{m}), 6.95-7.00(1 \mathrm{H}, \mathrm{m})$, $7.25-7.45(5 \mathrm{H}, \mathrm{m}), 7.85-7.89(1 \mathrm{H}, \mathrm{m}) .{ }^{19} \mathrm{~F}-\mathrm{NMR}\left(\mathrm{CDCl}_{3}\right) \delta:-27.7-$ $-27.8(1 \mathrm{~F}, \mathrm{~m}),-30.0--30.1(1 \mathrm{~F}, \mathrm{~m})$. MS $m / z(\%): 264\left(41, \mathrm{M}^{+}\right), 141$ (100). HRMS $m / z$ : 264.0384 (Calcd for $\mathrm{C}_{14} \mathrm{H}_{10} \mathrm{~F}_{2} \mathrm{OS}: 264.0240$ ). IR (neat) $\mathrm{cm}^{-1}: 1680$.

2',4'-Difluoro-2-[(4-methylphenyl)thio]acetophenone (5o): Colorless oil. Yield 99\% ${ }^{1} \mathrm{H}-\mathrm{NMR}\left(\mathrm{CDCl}_{3}\right) \delta: 2.31(3 \mathrm{H}, \mathrm{s}), 4.15(2 \mathrm{H}, \mathrm{s}), 6.85-6.90(1 \mathrm{H}$, $\mathrm{m}), 6.95-7.00(1 \mathrm{H}, \mathrm{m}), 7.08(2 \mathrm{H}, \mathrm{d}, J=7.8 \mathrm{~Hz}), 7.25(2 \mathrm{H}, \mathrm{d}, J=7.8 \mathrm{~Hz})$, 7.85-7.89 (1H, m). ${ }^{19} \mathrm{~F}-\mathrm{NMR}\left(\mathrm{CDCl}_{3}\right) \delta:-27.7--27.8(1 \mathrm{~F}, \mathrm{~m}),-30.0-$ -30.1 (1F, m). MS $m / z$ (\%): $278\left(46, \mathrm{M}^{+}\right), 141$ (100). HRMS $m / z: 278.0587$ (Calcd for $\mathrm{C}_{15} \mathrm{H}_{12} \mathrm{~F}_{2} \mathrm{OS}: 278.0577$ ). IR (neat) $\mathrm{cm}^{-1}: 1680$.

2-(Benzylthio)-2', $4^{\prime}$-difluoroacetophenone (5p): Colorless oil. Yield $68 \%$ ${ }^{1} \mathrm{H}-\mathrm{NMR}\left(\mathrm{CDCl}_{3}\right) \delta: 3.63(2 \mathrm{H}, \mathrm{s}), 3.64(2 \mathrm{H}, \mathrm{s}), 6.82-6.87(1 \mathrm{H}, \mathrm{m}), 6.90-$ $7.00(1 \mathrm{H}, \mathrm{m}), 7.27-7.34(5 \mathrm{H}, \mathrm{m}), 7.92-7.98(1 \mathrm{H}, \mathrm{m}) .{ }^{19} \mathrm{~F}-\mathrm{NMR}\left(\mathrm{CDCl}_{3}\right)$ $\delta:-30.7-30.8(1 \mathrm{~F}, \mathrm{~m}),-32.5-32.6(1 \mathrm{~F}, \mathrm{~m}) . \mathrm{MS} m / z(\%): 278(11$, $\mathrm{M}^{+}$), 141 (100). HRMS $m / z$ : 278.0591 (Calcd for $\mathrm{C}_{15} \mathrm{H}_{12} \mathrm{~F}_{2} \mathrm{OS}: 278.0577$ ). IR (neat) $\mathrm{cm}^{-1}: 1675$.

2',4'-Difluoro-2-[(4-methoxybenzyl)thio]acetophenone (5q): Colorless oil. Yield $33 \%{ }^{1} \mathrm{H}-\mathrm{NMR}\left(\mathrm{CDCl}_{3}\right) \delta: 3.63(2 \mathrm{H}, \mathrm{s}), 3.64(2 \mathrm{H}, \mathrm{s}), 3.79(3 \mathrm{H}, \mathrm{s})$, $6.83(2 \mathrm{H}, \mathrm{d}, J=8.8 \mathrm{~Hz}), 6.82-6.87(1 \mathrm{H}, \mathrm{m}), 6.90-7.00(1 \mathrm{H}, \mathrm{m}), 7.23(2 \mathrm{H}$, $\mathrm{d}, J=8.8 \mathrm{~Hz}), 7.92-7.98(1 \mathrm{H}, \mathrm{m}) .{ }^{19} \mathrm{~F}-\mathrm{NMR}\left(\mathrm{CDCl}_{3}\right) \delta:-30.7-30.8(1 \mathrm{~F}$, $\mathrm{m}),-32.5-32.6(1 \mathrm{~F}, \mathrm{~m})$. MS $\mathrm{m} / z(\%): 308\left(7, \mathrm{M}^{+}\right), 141$ (100). HRMS $\mathrm{m} / \mathrm{z}$ : 308.0678 (Calcd for $\mathrm{C}_{16} \mathrm{H}_{14} \mathrm{~F}_{2} \mathrm{O}_{2} \mathrm{~S}: 308.0682$ ). IR (neat) $\mathrm{cm}^{-1}: 1675$.

Preparation of 2-(2-Acetoxyethylthio)-2', $\mathbf{4}^{\prime}$-difluoroacetophenone (5I) from $5 \mathrm{~m}$ by Acetylation Acetic anhydride $(0.60 \mathrm{~g}, 5.88 \mathrm{mmol})$ was added to a solution of $2^{\prime}, 4^{\prime}$-difluoro-2-[(2-hydroxyethyl)thio]acetophenone $(\mathbf{5 m})$ $(1.14 \mathrm{~g}, 4.91 \mathrm{mmol})$ in pyridine $(10 \mathrm{ml})$. The mixture was stirred at $50^{\circ} \mathrm{C}$ for $3.0 \mathrm{~h}$. The reaction mixture was evaporated under reduced pressure. The residue was diluted ether. The etheral solution was washed with aqueous saturated copper sulfate, water, brine, dried over magnesium sulfate and evaporated under reduced pressure. Distillation of crude product at $164^{\circ} \mathrm{C}$ $(3 \mathrm{mmHg})$ gave $5 \mathbf{l}$ as a pale yellow oil $(1.31 \mathrm{~g}, 97 \%) .{ }^{1} \mathrm{H}-\mathrm{NMR}\left(\mathrm{CDCl}_{3}\right) \delta$ : $2.06(3 \mathrm{H}, \mathrm{s}), 2.76(2 \mathrm{H}, \mathrm{t}, J=6.4 \mathrm{~Hz}), 3.84\left(2 \mathrm{H}, \mathrm{d}, J_{\mathrm{HF}}=2.4 \mathrm{~Hz}\right), 4.25(2 \mathrm{H}, \mathrm{t}$, $J=6.4 \mathrm{~Hz}), 6.87-6.92(1 \mathrm{H}, \mathrm{m}), 6.98-7.03(1 \mathrm{H}, \mathrm{m}), 7.98-8.04(1 \mathrm{H}, \mathrm{m})$. ${ }^{19} \mathrm{~F}-\mathrm{NMR}\left(\mathrm{CDCl}_{3}\right) \delta:-24.9-25.0(1 \mathrm{~F}, \mathrm{~m}),-27.4-27.5(1 \mathrm{~F}, \mathrm{~m}) . \mathrm{MS}$ $m / z$ (\%): $274\left(5, \mathrm{M}^{+}\right), 141$ (100). HRMS m/z: 274.0470 (Calcd for $\mathrm{C}_{12} \mathrm{H}_{12} \mathrm{~F}_{2} \mathrm{O}_{3} \mathrm{~S}: 274.0476$ ). IR (neat) $\mathrm{cm}^{-1}: 1675$.

General Procedure for the Synthesis of Ethyl 2,2-Difluoro(substituted thio)acetate (6) $\mathrm{NaH}(55 \mathrm{mmol})$ was added to a solution of mercaptan $(50 \mathrm{mmol})$ in DMSO $(50 \mathrm{ml})$ at room temperature with stirring. After the mixture was stirred at the same temperature for $1 \mathrm{~h}$, ethyl bromodifluoroacetate $(55 \mathrm{mmol})$ was added to the solution at room temperature with stirring. The mixture was stirred at the same temperature for $15 \mathrm{~h}$. After addition of aqueous $\mathrm{NH}_{4} \mathrm{Cl}$, the mixture was extracted with $\mathrm{Et}_{2} \mathrm{O}$. The organic extract was washed with water and brine, dried over $\mathrm{MgSO}_{4}$, and evaporated under reduced pressure. The residue was chromatographed on a silica gel column using AcOEt- $n$-hexane $[1: 9(\mathrm{v} / \mathrm{v})]$ to give 6 .

Ethyl 2-(tert-Butylthio)-2,2-difluoroacetate (6f): Colorless oil. bp 55$56^{\circ} \mathrm{C} /(3 \mathrm{mmHg})$. Yield $40 \% .{ }^{1} \mathrm{H}-\mathrm{NMR}\left(\mathrm{CDCl}_{3}\right) \delta: 1.37(3 \mathrm{H}, \mathrm{t}, J=7.3 \mathrm{~Hz})$, $1.52(9 \mathrm{H}, \mathrm{s}), 4.35(2 \mathrm{H}, \mathrm{q}, J=7.3 \mathrm{~Hz}) .{ }^{19} \mathrm{~F}-\mathrm{NMR}\left(\mathrm{CDCl}_{3}\right) \delta:-3.4(2 \mathrm{~F}, \mathrm{~s}) . \mathrm{MS}$ $m / z: 212\left(0.6, \mathrm{M}^{+}\right), 57(100)$. HRMS $m / z: 212.0661$ (Calcd for $\mathrm{C}_{8} \mathrm{H}_{14} \mathrm{~F}_{2} \mathrm{O}_{2} \mathrm{~S}$ : 212.0682). IR (neat) $\mathrm{cm}^{-1}: 1770$.

Ethyl 2,2-Difluoro-2-(phenylthio)acetate (6n): Colorless oil. bp $109^{\circ} \mathrm{C} /$ $(10 \mathrm{mmHg})$. Yield $48 \% .{ }^{1} \mathrm{H}-\mathrm{NMR}\left(\mathrm{CDCl}_{3}\right) \delta: 1.25(3 \mathrm{H}, \mathrm{t}, J=7.3 \mathrm{~Hz}), 4.24$ $(2 \mathrm{H}, \mathrm{q}, J=7.3 \mathrm{~Hz}), 7.37-7.40(2 \mathrm{H}, \mathrm{m}), 7.41-7.46(1 \mathrm{H}, \mathrm{m}), 7.60-7.63$ $(2 \mathrm{H}, \mathrm{m}) .{ }^{19} \mathrm{~F}-\mathrm{NMR}\left(\mathrm{CDCl}_{3}\right) \delta:-6.5(2 \mathrm{~F}, \mathrm{~s}) . \mathrm{MS} m / z: 232\left(71, \mathrm{M}^{+}\right), 159$ (100). HRMS $m / z$ : 232.0360 (Calcd for $\mathrm{C}_{10} \mathrm{H}_{10} \mathrm{~F}_{2} \mathrm{O}_{2} \mathrm{~S}: 232.0370$ ). IR (neat) $\mathrm{cm}^{-1}: 1765$.

Ethyl 2,2-Difluoro-2-[(4-methylphenyl)thio]acetate (6o): Colorless oil. Yield 47\%. ${ }^{1} \mathrm{H}-\mathrm{NMR}\left(\mathrm{CDCl}_{3}\right) \delta: 1.27(3 \mathrm{H}, \mathrm{t}, J=7.3 \mathrm{~Hz}), 2.38(3 \mathrm{H}, \mathrm{s}), 4.25$ $(2 \mathrm{H}, \mathrm{q}, J=7.3 \mathrm{~Hz}), 7.20(2 \mathrm{H}, \mathrm{d}, J=8.3 \mathrm{~Hz}), 7.49(2 \mathrm{H}, \mathrm{d}, J=8.3 \mathrm{~Hz}) .{ }^{19} \mathrm{~F}-$ NMR $\left(\mathrm{CDCl}_{3}\right) \delta$ : $-6.9(2 \mathrm{~F}, \mathrm{~s})$. MS $m / z(\%): 246\left(88, \mathrm{M}^{+}\right), 173(100)$. HRMS $m / z$ : 246.0533 (Calcd for $\mathrm{C}_{11} \mathrm{H}_{12} \mathrm{~F}_{2} \mathrm{O}_{2} \mathrm{~S}: 246.0526$ ). IR (neat) $\mathrm{cm}^{-1}$ : 1770 .

Ethyl 2-(Benzylthio)-2,2-difluoroacetate (6p): Colorless oil. bp 116$117^{\circ} \mathrm{C} /(3 \mathrm{mmHg})$. Yield $31 \% .{ }^{1} \mathrm{H}-\mathrm{NMR}\left(\mathrm{CDCl}_{3}\right) \delta: 1.33(3 \mathrm{H}, \mathrm{t}, J=7.3 \mathrm{~Hz})$, $4.12(2 \mathrm{H}, \mathrm{s}), 4.30(2 \mathrm{H}, \mathrm{q}, J=7.3 \mathrm{~Hz}), 7.27-7.34(5 \mathrm{H}, \mathrm{m}) .{ }^{19} \mathrm{~F}-\mathrm{NMR}$
$\left(\mathrm{CDCl}_{3}\right) \delta:-7.3(2 \mathrm{~F}, \mathrm{~s})$. MS $m / z: 246\left(4, \mathrm{M}^{+}\right), 91$ (100). HRMS $m / z$ : 246.0523 (Calcd for $\mathrm{C}_{11} \mathrm{H}_{12} \mathrm{~F}_{2} \mathrm{O}_{2} \mathrm{~S}: 246.0526$ ). IR (neat) $\mathrm{cm}^{-1}: 1770$.

Ethyl 2,2-Difluoro-2-[(4-methoxybenzyl)thio]acetate (6q): Colorless oil. Yield 53\%. ${ }^{1} \mathrm{H}-\mathrm{NMR}\left(\mathrm{CDCl}_{3}\right) \delta: 1.34(3 \mathrm{H}, \mathrm{t}, J=7.3 \mathrm{~Hz}), 3.73(3 \mathrm{H}, \mathrm{s}), 4.08$ $(2 \mathrm{H}, \mathrm{s}), 4.32(2 \mathrm{H}, \mathrm{q}, J=7.3 \mathrm{~Hz}), 6.86(2 \mathrm{H}, \mathrm{d}, J=8.8 \mathrm{~Hz}), 7.26(2 \mathrm{H}, \mathrm{d}$, $J=8.8 \mathrm{~Hz}) \cdot{ }^{19} \mathrm{~F}-\mathrm{NMR}\left(\mathrm{CDCl}_{3}\right) \delta:-7.3(2 \mathrm{~F}, \mathrm{~s}) . \mathrm{MS} m / z(\%): 276\left(7, \mathrm{M}^{+}\right)$, 121 (100). HRMS m/z: 276.0609 (Calcd for $\mathrm{C}_{12} \mathrm{H}_{14} \mathrm{~F}_{2} \mathrm{O}_{3} \mathrm{~S}: 276.0632$ ). IR (neat) $\mathrm{cm}^{-1}: 1765$.

General Procedure of Synthesis of 2,2-Difluoro-2-(substituted thio)acetophenone (3). (A) General Procedure via Fluorination A solution of $5(50 \mathrm{mmol})$ in 1,1,2-trichloroethane (TCE) $(10 \mathrm{ml})$ was added dropwise into the suspension of $\mathrm{N}$-fluoro-2,4,6-trimethylpyridinium triflate (FPT300) $(150 \mathrm{mmol})$ and zinc bromide $(25 \mathrm{mmol})$ in TCE $(100 \mathrm{ml})$ for $10 \mathrm{~min}$ at $80^{\circ} \mathrm{C}-100^{\circ} \mathrm{C}$. The mixture was stirred at $105^{\circ} \mathrm{C}$. After addition of water, the mixture was extracted with $\mathrm{CHCl}_{3}$. The organic extract was washed with water and brine, dried over $\mathrm{MgSO}_{4}$, and evaporated under reduced pressure. The residue was chromatographed on a silica gel column using AcOEt- $n$-hexane $[1: 9(\mathrm{v} / \mathrm{v})]$ or distilled under reduced pressure to give 3 .

2-(Methylthio)-2,2,2',4'-tetrafluoroacetophenone (3a): Colorless oil. bp $96{ }^{\circ} \mathrm{C} /(5 \mathrm{mmHg})$. Yield $82 \% .{ }^{1} \mathrm{H}-\mathrm{NMR}\left(\mathrm{CDCl}_{3}\right) \delta: 2.35(3 \mathrm{H}, \mathrm{s}), 6.91-7.02$ $(2 \mathrm{H}, \mathrm{m}), 7.95-8.01(1 \mathrm{H}, \mathrm{m}) .{ }^{19} \mathrm{~F}-\mathrm{NMR}\left(\mathrm{CDCl}_{3}\right) \delta:-9.4(2 \mathrm{~F}, \mathrm{~d}, J=15 \mathrm{~Hz})$, $-23.0-23.1(1 \mathrm{~F}, \mathrm{~m}),-26.0-26.1(1 \mathrm{~F}, \mathrm{~m})$. MS $m / z(\%): 238\left(1, \mathrm{M}^{+}\right)$, 141 (100). HRMS $m / z$ : 238.0062 (Calcd for $\mathrm{C}_{9} \mathrm{H}_{6} \mathrm{~F}_{4} \mathrm{OS}: 238.0076$ ). IR (neat) $\mathrm{cm}^{-1}: 1700$.

2-(Ethylthio)-2,2,2',4'-tetrafluoroacetophenone (3b): Colorless oil. bp $105^{\circ} \mathrm{C} /(7 \mathrm{mmHg})$. Yield $85 \% .{ }^{1} \mathrm{H}-\mathrm{NMR}\left(\mathrm{CDCl}_{3}\right) \delta: 1.37(3 \mathrm{H}, \mathrm{t}, J=7.8 \mathrm{~Hz})$, $2.92(2 \mathrm{H}, \mathrm{q}, J=7.8 \mathrm{~Hz}), 6.90-7.02(2 \mathrm{H}, \mathrm{m}), 7.95-8.01(1 \mathrm{H}, \mathrm{m}) .{ }^{19} \mathrm{~F}-\mathrm{NMR}$ $\left(\mathrm{CDCl}_{3}\right) \delta:-6.4(2 \mathrm{~F}, \mathrm{~d}, J=14 \mathrm{~Hz}),-23.0-23.1(1 \mathrm{~F}, \mathrm{~m}),-26.1--26.2$ (1F, m). MS $m / z(\%): 252\left(0.3, \mathrm{M}^{+}\right), 141$ (100). HRMS $m / z: 252.0229$ (Calcd for $\mathrm{C}_{10} \mathrm{H}_{8} \mathrm{~F}_{4} \mathrm{OS}: 252.0232$ ). IR (neat) $\mathrm{cm}^{-1}: 1715$.

2-(Propylthio)-2,2,2',4'-tetrafluoroacetophenone (3c): Colorless oil. bp $102{ }^{\circ} \mathrm{C} /(4 \mathrm{mmHg})$. Yield $31 \% .{ }^{1} \mathrm{H}-\mathrm{NMR}\left(\mathrm{CDCl}_{3}\right) \delta: 1.02(3 \mathrm{H}, \mathrm{t}, J=7.3 \mathrm{~Hz})$, $1.70-1.75(2 \mathrm{H}, \mathrm{m}), 2.87(2 \mathrm{H}, \mathrm{t}, J=7.3 \mathrm{~Hz}), 6.91-6.97(1 \mathrm{H}, \mathrm{m}), 6.98-$ $7.02(1 \mathrm{H}, \mathrm{m}), 7.95-8.00(1 \mathrm{H}, \mathrm{m}) .{ }^{19} \mathrm{~F}-\mathrm{NMR}\left(\mathrm{CDCl}_{3}\right) \delta:-6.4(2 \mathrm{~F}, \mathrm{~d}$, $J=14 \mathrm{~Hz}),-23.1--23.2(1 \mathrm{~F}, \mathrm{~m}),-26.1-26.2(1 \mathrm{~F}, \mathrm{~m})$. MS $\mathrm{m} / z(\%)$ : $266\left(0.5, \mathrm{M}^{+}\right), 141(100)$. HRMS $m / z$ : 266.0365 (Calcd for $\mathrm{C}_{11} \mathrm{H}_{10} \mathrm{~F}_{4} \mathrm{OS}$ : 266.0389). IR (neat) $\mathrm{cm}^{-1}: 1710$.

2-(Cyclopropylthio)-2,2,2',4'-tetrafluoroacetophenone (3d): Colorless oil. bp $115^{\circ} \mathrm{C} /(4 \mathrm{mmHg})$. Yield $72 \%$. ${ }^{1} \mathrm{H}-\mathrm{NMR}\left(\mathrm{CDCl}_{3}\right) \delta: 0.71-0.75(2 \mathrm{H}, \mathrm{m})$, $0.99-1.04(2 \mathrm{H}, \mathrm{m}), 2.06-2.10(1 \mathrm{H}, \mathrm{m}), 6.91-7.02(2 \mathrm{H}, \mathrm{m}), 7.96-8.02$ $(1 \mathrm{H}, \mathrm{m}) .{ }^{19} \mathrm{~F}-\mathrm{NMR}\left(\mathrm{CDCl}_{3}\right) \delta:-7.5(2 \mathrm{~F}, \mathrm{~d}, J=12 \mathrm{~Hz}),-23.0-23.1(1 \mathrm{~F}$, $\mathrm{m}),-26.0-26.1(1 \mathrm{~F}, \mathrm{~m})$. MS $m / z(\%): 264\left(0.2, \mathrm{M}^{+}\right), 141$ (100). HRMS $m / z: 264.0206$ (Calcd for $\mathrm{C}_{11} \mathrm{H}_{8} \mathrm{~F}_{4} \mathrm{OS}: 264.0232$ ). IR (neat) $\mathrm{cm}^{-1}: 1715$.

2-(Butylthio)-2,2,2',4'-tetrafluoroacetophenone (3e): Colorless oil. bp $116^{\circ} \mathrm{C} /(3 \mathrm{mmHg})$. Yield $24 \% .{ }^{1} \mathrm{H}-\mathrm{NMR}\left(\mathrm{CDCl}_{3}\right) \delta: 0.93(3 \mathrm{H}, \mathrm{t}, J=7.3 \mathrm{~Hz})$, $1.40-1.48(2 \mathrm{H}, \mathrm{m}), 1.64-1.71(2 \mathrm{H}, \mathrm{m}), 2.88(2 \mathrm{H}, \mathrm{t}, J=7.3 \mathrm{~Hz}), 6.91-$ $7.02(2 \mathrm{H}, \mathrm{m}), 7.95-8.01(1 \mathrm{H}, \mathrm{m}) .{ }^{19} \mathrm{~F}-\mathrm{NMR}\left(\mathrm{CDCl}_{3}\right) \delta:-6.4(2 \mathrm{~F}, \mathrm{~d}$, $J=13 \mathrm{~Hz}),-23.1-23.2(1 \mathrm{~F}, \mathrm{~m}),-26.1-26.2(1 \mathrm{~F}, \mathrm{~m})$. MS $\mathrm{m} / \mathrm{z}(\%)$ : $280\left(0.5, \mathrm{M}^{+}\right.$), 141 (100). HRMS m/z: 280.0562 (Calcd for $\mathrm{C}_{12} \mathrm{H}_{12} \mathrm{~F}_{4} \mathrm{OS}$ : 280.0545). IR (neat) $\mathrm{cm}^{-1}: 1715$.

2-(Pentylthio)-2,2,2',4'-tetrafluoroacetophenone (3g): Colorless oil. bp $132{ }^{\circ} \mathrm{C} /(4 \mathrm{mmHg})$. Yield $37 \% .{ }^{1} \mathrm{H}-\mathrm{NMR}\left(\mathrm{CDCl}_{3}\right) \delta: 0.93(3 \mathrm{H}, \mathrm{t}, J=7.3 \mathrm{~Hz})$, $1.45-1.80(6 \mathrm{H}, \mathrm{m}), 2.88(2 \mathrm{H}, \mathrm{t}, J=7.3 \mathrm{~Hz}), 6.91-7.02(2 \mathrm{H}, \mathrm{m}), 7.95-$ $8.01(1 \mathrm{H}, \mathrm{m}) .{ }^{19} \mathrm{~F}-\mathrm{NMR}\left(\mathrm{CDCl}_{3}\right) \delta:-6.3(2 \mathrm{~F}, \mathrm{~d}, J=14 \mathrm{~Hz}),-23.0-23.1$ (1F, m), $-26.1--26.2$ (1F, m). MS $m / z$ (\%): $294\left(0.3, \mathrm{M}^{+}\right), 141$ (100). HRMS $m / z$ : 294.3088 (Calcd for $\mathrm{C}_{13} \mathrm{H}_{14} \mathrm{~F}_{4}$ OS: 294.3093). IR (neat) $\mathrm{cm}^{-1}$ : 1715 .

2,2-Difluoro-2-(methylthio)acetophenone (3h): Colorless oil. bp $87^{\circ} \mathrm{C} /$ $(4 \mathrm{mmHg})$. Yield $82 \% .{ }^{1} \mathrm{H}-\mathrm{NMR}\left(\mathrm{CDCl}_{3}\right) \delta: 2.36(3 \mathrm{H}, \mathrm{s}), 7.50(2 \mathrm{H}, \mathrm{t}$, $J=7.8 \mathrm{~Hz}), 7.65(1 \mathrm{H}, \mathrm{dd}, J=7.8,1.5 \mathrm{~Hz}), 8.14(2 \mathrm{H}, \mathrm{dd}, J=7.8,1.5 \mathrm{~Hz}) .{ }^{19} \mathrm{~F}-$ $\operatorname{NMR}\left(\mathrm{CDCl}_{3}\right) \delta:-3.3(2 \mathrm{~F}, \mathrm{~s})$. MS $m / z(\%): 202\left(3, \mathrm{M}^{+}\right), 105(100)$. HRMS $\mathrm{m} / \mathrm{z}: 202.0256$ (Calcd for $\mathrm{C}_{9} \mathrm{H}_{8} \mathrm{~F}_{2} \mathrm{OS}: 202.0264$ ). IR (neat) $\mathrm{cm}^{-1}: 1705$.

2-(Methylthio)-2,2,4'-trifluoroacetophenone (3i): Colorless oil. bp $79^{\circ} \mathrm{C} /$ $(3 \mathrm{mmHg})$. Yield $45 \% .{ }^{1} \mathrm{H}-\mathrm{NMR}\left(\mathrm{CDCl}_{3}\right) \delta: 2.37(3 \mathrm{H}, \mathrm{s}), 7.15-7.20(2 \mathrm{H}$, m), $8.15-8.20(2 \mathrm{H}, \mathrm{m}) .{ }^{19} \mathrm{~F}-\mathrm{NMR}\left(\mathrm{CDCl}_{3}\right) \delta:-5.9(2 \mathrm{~F}, \mathrm{~s}),-25.7(1 \mathrm{~F}, \mathrm{~s})$. MS $m / z(\%): 220\left(1, \mathrm{M}^{+}\right), 123$ (100). HRMS $m / z: 220.0175$ (Calcd for $\left.\mathrm{C}_{9} \mathrm{H}_{7} \mathrm{~F}_{3} \mathrm{OS}: 220.0170\right)$. IR (neat) $\mathrm{cm}^{-1}: 1705$.

2,2-Difluoro-2-(methylthio)-4' -(trifluoromethyl)acetophenone (3j): Colorless oil. bp $107^{\circ} \mathrm{C} /(5 \mathrm{mmHg})$. Yield $60 \% .{ }^{1} \mathrm{H}-\mathrm{NMR}\left(\mathrm{CDCl}_{3}\right) \delta: 2.38(3 \mathrm{H}, \mathrm{s})$, $7.77(2 \mathrm{H}, \mathrm{d}, J=8.3 \mathrm{~Hz}), 8.25(2 \mathrm{H}, \mathrm{d}, J=8.3 \mathrm{~Hz}){ }^{19} \mathrm{~F}-\mathrm{NMR}\left(\mathrm{CDCl}_{3}\right) \delta: 12.3$ (3F, s), -6.8 (2F, s). MS $m / z(\%): 270\left(3, \mathrm{M}^{+}\right), 173(100)$. HRMS $m / z$ : 270.0093 (Calcd for $\left.\mathrm{C}_{10} \mathrm{H}_{7} \mathrm{~F}_{5} \mathrm{OS}: 270.0138\right)$. IR (neat) $\mathrm{cm}^{-1}: 1715$.

2',4'-Dichloro-2,2-difluoro-2-(methylthio)acetophenone (3k): Colorless 
oil. Yield 72\%. ${ }^{1} \mathrm{H}-\mathrm{NMR}\left(\mathrm{CDCl}_{3}\right) \delta: 2.35(3 \mathrm{H}, \mathrm{s}), 7.34(1 \mathrm{H}, \mathrm{dd}, J=8.3$, $2.0 \mathrm{~Hz}), 7.53(1 \mathrm{H}, \mathrm{d}, J=2.0 \mathrm{~Hz}), 7.69(1 \mathrm{H}, \mathrm{d}, J=8.3 \mathrm{~Hz}) .{ }^{19} \mathrm{~F}-\mathrm{NMR}\left(\mathrm{CDCl}_{3}\right)$ $\delta:-10.1(2 \mathrm{~F}, \mathrm{~s})$. MS $\mathrm{m} / \mathrm{z}(\%): 270\left(1, \mathrm{M}^{+}\right), 173(100)$. HRMS $\mathrm{m} / \mathrm{z}$ : 269.9459 (Calcd for $\mathrm{C}_{9} \mathrm{H}_{6} \mathrm{Cl}_{2} \mathrm{~F}_{2} \mathrm{OS}: 269.9485$ ). IR (neat) $\mathrm{cm}^{-1}: 1725$.

2-(2-Acetoxyethylthio)-2,2,2',4'-tetrafluoroacetophenone (31): Colorless oil. bp $151{ }^{\circ} \mathrm{C} /(3 \mathrm{mmHg})$. Yield $45 \% .{ }^{1} \mathrm{H}-\mathrm{NMR}\left(\mathrm{CDCl}_{3}\right) \delta: 2.07(3 \mathrm{H}, \mathrm{s})$, $3.14(2 \mathrm{H}, \mathrm{t}, J=6.4 \mathrm{~Hz}), 4.31(2 \mathrm{H}, \mathrm{t}, J=6.4 \mathrm{~Hz}), 6.92-7.03(2 \mathrm{H}, \mathrm{m}), 7.94$ $7.99(1 \mathrm{H}, \mathrm{m}) .{ }^{19} \mathrm{~F}-\mathrm{NMR}\left(\mathrm{CDCl}_{3}\right) \delta:-5.4(2 \mathrm{~F}, \mathrm{~d}, J=15 \mathrm{~Hz}),-22.4-22.5$ $(1 \mathrm{~F}, \mathrm{~m}),-25.5-25.6(1 \mathrm{~F}, \mathrm{~m}) . \mathrm{MS} m / z$ (\%): 250 (11, $\left.\mathrm{M}^{+}-\mathrm{AcOH}\right), 141$ (100). HRMS $m / z: 250.0032$ (Calcd for $\mathrm{C}_{10} \mathrm{H}_{6} \mathrm{~F}_{4} \mathrm{OS}: 250.0076$ ). IR (neat) $\mathrm{cm}^{-1}: 1744,1712$.

(B) General Procedure of Synthesis of 2,2-Difluoro-2-(substituted thio)acetophenone (3) via Lithiation A solution of $n$-BuLi ( $1.37 \mathrm{M}$ solution in $n$-hexane; $20 \mathrm{mmol}$ ) was added to a solution of bromobenzene (7) $(20 \mathrm{mmol})$ in $\mathrm{Et}_{2} \mathrm{O}(100 \mathrm{ml})$ with stirring at $-78^{\circ} \mathrm{C}$ under $\mathrm{Ar}$ atmosphere. The mixture was stirred at the same temperature for $0.5 \mathrm{~h}$. A solution of ethyl difluoroacetate $(6)(20 \mathrm{mmol})$ in $\mathrm{Et}_{2} \mathrm{O}(10 \mathrm{ml})$ was added to the solution with stirring at $-78^{\circ} \mathrm{C}$ and stirred at the same temperature for $1 \mathrm{~h}$ and at room temperature for $1 \mathrm{~h}$. After addition of aqueous $\mathrm{NH}_{4} \mathrm{Cl}$, the mixture was extracted with AcOEt. The organic extract was washed with water and brine, dried over $\mathrm{MgSO}_{4}$, and evaporated under reduced pressure. The residue was chromatographed on a silica gel column using AcOEt- $n$-hexane $[1: 8(\mathrm{v} / \mathrm{v})]$ to give 3 .

2-(tert-Butylthio)-2,2,2',4'-tetrafluoroacetophenone (3f): Colorless oil. Yield 68\%. ${ }^{1} \mathrm{H}-\mathrm{NMR}\left(\mathrm{CDCl}_{3}\right) \delta: 1.56(9 \mathrm{H}, \mathrm{s}), 6.72-7.01(2 \mathrm{H}, \mathrm{m}), 7.97-$ $8.03(1 \mathrm{H}, \mathrm{m}) .{ }^{19} \mathrm{~F}-\mathrm{NMR}\left(\mathrm{CDCl}_{3}\right) \delta:-2.01(2 \mathrm{~F}, \mathrm{~d}, J=9 \mathrm{~Hz}),-23.2--23.3$ (1F, m), $-26.4-26.5(1 \mathrm{~F}, \mathrm{~m})$. MS $m / z: 280\left(2, \mathrm{M}^{+}\right), 57$ (100). HRMS $m / z: 280.0574$ (Calcd for $\mathrm{C}_{12} \mathrm{H}_{12} \mathrm{~F}_{4} \mathrm{OS}: 280.0545$ ). IR (neat) $\mathrm{cm}^{-1}: 1715$.

2-(Phenylthio)-2,2,2',4'-tetrafluoroacetophenone (3n): Colorless oil. Yield $61 \%$. ${ }^{1} \mathrm{H}-\mathrm{NMR}\left(\mathrm{CDCl}_{3}\right) \delta: 6.90-7.00(2 \mathrm{H}, \mathrm{m}), 7.26-7.45(2 \mathrm{H}, \mathrm{m}), 7.46-$ $7.48(1 \mathrm{H}, \mathrm{m}), 7.58-7.61(2 \mathrm{H}, \mathrm{m}), 7.91-7.97(1 \mathrm{H}, \mathrm{m}) .{ }^{19} \mathrm{~F}-\mathrm{NMR}\left(\mathrm{CDCl}_{3}\right)$ $\delta:-4.4(2 \mathrm{~F}, \mathrm{~d}, J=14 \mathrm{~Hz}),-22.7-22.8(1 \mathrm{~F}, \mathrm{~m}),-25.8-25.9(1 \mathrm{~F}, \mathrm{~m})$. MS $m / z: 300\left(9, \mathrm{M}^{+}\right), 141$ (100). HRMS $\mathrm{m} / z: 300.0228$ (Calcd for $\mathrm{C}_{14} \mathrm{H}_{8} \mathrm{~F}_{4} \mathrm{OS}: 300.0232$ ). IR (neat) $\mathrm{cm}^{-1}: 1710$.

2-[(4-Methylphenyl)thio]-2,2,2',4'-tetrafluoroacetophenone (3o): Colorless oil. Yield $66 \%$. ${ }^{1} \mathrm{H}-\mathrm{NMR}\left(\mathrm{CDCl}_{3}\right) \delta: 2.37(3 \mathrm{H}, \mathrm{s}), 6.90-7.00(2 \mathrm{H}, \mathrm{m})$, $7.19(2 \mathrm{H}, \mathrm{d}, J=8.3 \mathrm{~Hz}), 7.46(2 \mathrm{H}, \mathrm{d}, J=8.3 \mathrm{~Hz}), 7.92-7.98(1 \mathrm{H}, \mathrm{m}) .{ }^{19} \mathrm{~F}-$ $\operatorname{NMR}\left(\mathrm{CDCl}_{3}\right) \delta:-5.0(2 \mathrm{~F}, \mathrm{~d}, J=14 \mathrm{~Hz}),-22.9-23.0(1 \mathrm{~F}, \mathrm{~m}),-25.8-$ -26.0 (1F, m). MS $m / z(\%): 314\left(18, \mathrm{M}^{+}\right), 141$ (100). HRMS $m / z: 314.0403$ (Calcd for $\mathrm{C}_{15} \mathrm{H}_{10} \mathrm{~F}_{4} \mathrm{OS}: 314.0389$ ). IR (neat) $\mathrm{cm}^{-1}: 1660$.

2-(Benzylthio)-2,2,2',4'-tetrafluoroacetophenone (3p): Colorless oil. Yield $84 \% .{ }^{1} \mathrm{H}-\mathrm{NMR}\left(\mathrm{CDCl}_{3}\right) \delta: 4.12(2 \mathrm{H}, \mathrm{s}), 6.90-7.00(2 \mathrm{H}, \mathrm{m}), 7.26-$ $7.35(5 \mathrm{H}, \mathrm{m}), 7.26-7.35(1 \mathrm{H}, \mathrm{m}) .{ }^{19} \mathrm{~F}-\mathrm{NMR}\left(\mathrm{CDCl}_{3}\right) \delta:-6.3(2 \mathrm{~F}, \mathrm{~d}$, $J=15 \mathrm{~Hz}),-22.8-22.9(1 \mathrm{~F}, \mathrm{~m}),-25.8-25.9(1 \mathrm{~F}, \mathrm{~m})$. MS $\mathrm{m} / \mathrm{z}: 314$ $\left(0.3, \mathrm{M}^{+}\right), 141(100)$. HRMS $m / z$ : 314.0387 (Calcd for $\mathrm{C}_{15} \mathrm{H}_{10} \mathrm{~F}_{4} \mathrm{OS}$ : 314.0388). IR (neat) $\mathrm{cm}^{-1}: 1715$.

2-[(4-Methoxybenzyl)thio]-2,2,2', $4^{\prime}$-tetrafluoroacetophenone (3q): Colorless oil. Yield 33\%. ${ }^{1} \mathrm{H}-\mathrm{NMR}\left(\mathrm{CDCl}_{3}\right) \delta: 3.79(3 \mathrm{H}, \mathrm{s}), 4.09(2 \mathrm{H}, \mathrm{s}), 6.84$ $(2 \mathrm{H}, \mathrm{d}, J=8.8 \mathrm{~Hz}), 6.89-7.00(2 \mathrm{H}, \mathrm{m}), 7.25(2 \mathrm{H}, \mathrm{d}, J=8.8 \mathrm{~Hz}), 7.92-7.98$ $(1 \mathrm{H}, \mathrm{m}) .{ }^{19} \mathrm{~F}-\mathrm{NMR}\left(\mathrm{CDCl}_{3}\right) \delta:-6.3(2 \mathrm{~F}, \mathrm{~d}, J=14 \mathrm{~Hz}),-22.8--22.9(1 \mathrm{~F}$, $\mathrm{m}),-25.8-26.0(1 \mathrm{~F}, \mathrm{~m})$. MS $m / z(\%): 344\left(3, \mathrm{M}^{+}\right), 121$ (100). HRMS $\mathrm{m} / z$ : 344.0474 (Calcd for $\left.\mathrm{C}_{16} \mathrm{H}_{12} \mathrm{~F}_{4} \mathrm{O}_{2} \mathrm{~S}: 344.0494\right)$. IR (neat) $\mathrm{cm}^{-1}: 1710$.

(C) Synthesis of 2,2-Difluoro-2-(methylthio)acetophenone (3h) via Methylthiolation Sodium methanethiolate $(60 \mathrm{mmol})$ was added to a solution of 2-Chloro-2,2-difluoroacetophenone $(50 \mathrm{mmol})$ in DMSO $(150 \mathrm{ml})$ at room temperature with stirring. The mixture was stirred at room temperature for $15 \mathrm{~h}$. After addition of water, the mixture was extracted with $\mathrm{Et}_{2} \mathrm{O}$. The organic extract was washed with water and brine, dried over $\mathrm{MgSO}_{4}$, and evaporated under reduced pressure. The residue was chromatographed on a silica gel column using AcOEt- $n$-hexane $[1: 9(\mathrm{v} / \mathrm{v})]$ or distilled under reduced pressure to give $\mathbf{3 h}$. (yield $49 \%$ )

General Procedure for the Synthesis of 2-Aryl-2-[difluoro(substituted thio)methyl]oxirane (8) from 3 with Trimethylsulfoxonium Iodide (TMSI) TMSI (5.5 mmol) was added to a suspension of $\mathrm{NaH}(60 \%$ in oil; $6 \mathrm{mmol})$ in DMSO $(30 \mathrm{ml})$ in tetrahydrofuran (THF) $(10 \mathrm{ml})$. After stirring at room temperature for $1 \mathrm{~h}$, the mixture was added to $3(5 \mathrm{mmol})$ in THF $(10 \mathrm{ml})$ at $0{ }^{\circ} \mathrm{C}$ with stirring. The mixture was stirred at the same temperature for $1 \mathrm{~h}$. After addition of aqueous $\mathrm{NaHCO}_{3}$, the mixture was extracted with AcOEt. The organic extract was washed with water and brine, dried over $\mathrm{MgSO}_{4}$, and evaporated under reduced pressure. The residue was chromatographed on a silica gel column using $n$-hexane-AcOEt $[9: 1(\mathrm{v} / \mathrm{v})]$ to give 8 .

2-[Difluoro(methylthio)methyl]-2-(2,4-difluorophenyl)oxirane (8a): Colorless oil. Yield $46 \% .{ }^{1} \mathrm{H}-\mathrm{NMR}\left(\mathrm{CDCl}_{3}\right) \delta: 2.28(3 \mathrm{H}, \mathrm{s}), 2.98-3.00(1 \mathrm{H}$, $\mathrm{m}), 3.48-3.50(1 \mathrm{H}, \mathrm{m}) .6 .81-6.87(1 \mathrm{H}, \mathrm{m}), 6.89-6.95(1 \mathrm{H}, \mathrm{m}), 7.51-$ $7.56(1 \mathrm{H}, \mathrm{m}) .{ }^{19} \mathrm{~F}-\mathrm{NMR}\left(\mathrm{CDCl}_{3}\right) \delta:-8.7(1 \mathrm{~F}, \mathrm{dd}, J=9,214 \mathrm{~Hz}),-9.5(1 \mathrm{~F}$, dd, $J=7,214 \mathrm{~Hz}),-31.4-31.5(1 \mathrm{~F}, \mathrm{~m}),-33.2--33.3(1 \mathrm{~F}, \mathrm{~m}) . \mathrm{MS} \mathrm{m} / \mathrm{z}$ (\%): $252\left(5, \mathrm{M}^{+}\right), 127(100)$. HRMS m/z: 252.0259 (Calcd for $\mathrm{C}_{10} \mathrm{H}_{8} \mathrm{~F}_{4} \mathrm{OS}$ : 252.0232). IR (neat) $\mathrm{cm}^{-1}: 1270$.

2-[Difluoro(ethylthio)methyl]-2-(2,4-difluorophenyl)oxirane (8b): Colorless oil. Yield $90 \% .{ }^{1} \mathrm{H}-\mathrm{NMR}\left(\mathrm{CDCl}_{3}\right) \delta: 1.30(3 \mathrm{H}, \mathrm{t}, J=7.3 \mathrm{~Hz}), 2.83-2.90$ $(2 \mathrm{H}, \mathrm{m}), 2.97-3.00(1 \mathrm{H}, \mathrm{m}), 3.48-3.50(1 \mathrm{H}, \mathrm{m}) .6 .81-6.90(1 \mathrm{H}, \mathrm{m})$, $6.91-6.94(1 \mathrm{H}, \mathrm{m}), 7.51-7.55(1 \mathrm{H}, \mathrm{m}) .{ }^{19} \mathrm{~F}-\mathrm{NMR}\left(\mathrm{CDCl}_{3}\right) \delta:-5.6(1 \mathrm{~F}$, dd, $J=9,214 \mathrm{~Hz}),-6.9(1 \mathrm{~F}, \mathrm{dd}, J=7,214 \mathrm{~Hz}),-31.4-31.5(1 \mathrm{~F}, \mathrm{~m})$, $-33.2-33.3$ (1F, m). MS $m / z(\%): 266\left(2, \mathrm{M}^{+}\right), 127(100)$. HRMS $m / z$ : 266.0401 (Calcd for $\mathrm{C}_{11} \mathrm{H}_{10} \mathrm{~F}_{4} \mathrm{OS}: 266.0389$ ). IR (neat) $\mathrm{cm}^{-1}: 1240$.

2-(2,4-Difluorophenyl)-2-[difluoro(propylthio)methyl]oxirane (8c): Colorless oil. Yield 46\%. ${ }^{1} \mathrm{H}-\mathrm{NMR}\left(\mathrm{CDCl}_{3}\right) \delta: 0.97(3 \mathrm{H}, \mathrm{t}, J=7.3 \mathrm{~Hz}), 1.58-1.69$ $(2 \mathrm{H}, \mathrm{m}), 2.81(2 \mathrm{H}, \mathrm{dt}, J=2.0,7.3 \mathrm{~Hz}), 2.98-3.00(1 \mathrm{H}, \mathrm{m}), 3.49-3.51(1 \mathrm{H}$, m). $6.81-6.87(1 \mathrm{H}, \mathrm{m}), 6.90-6.94(1 \mathrm{H}, \mathrm{m}), 7.51-7.57(1 \mathrm{H}, \mathrm{m}) .{ }^{19} \mathrm{~F}-\mathrm{NMR}$ $\left(\mathrm{CDCl}_{3}\right) \delta:-5.5(1 \mathrm{~F}, \mathrm{dd}, J=10,214 \mathrm{~Hz}),-6.8(1 \mathrm{~F}, \mathrm{dd}, J=7,214 \mathrm{~Hz})$, $-31.5-31.6(1 \mathrm{~F}, \mathrm{~m}),-33.2--33.3(1 \mathrm{~F}, \mathrm{~m})$. MS $m / z(\%): 280\left(16, \mathrm{M}^{+}\right)$, 155 (100). HRMS m/z: 280.0579 (Calcd for $\mathrm{C}_{12} \mathrm{H}_{12} \mathrm{~F}_{4} \mathrm{OS}: 280.0545$ ). IR (neat) $\mathrm{cm}^{-1}: 1245$.

2-[(Cyclopropylthio)difluoromethyl]-2-(2,4-difluorophenyl)oxirane (8d): Colorless oil. Yield $86 \% .{ }^{1} \mathrm{H}-\mathrm{NMR}\left(\mathrm{CDCl}_{3}\right) \delta: 0.65-0.70(2 \mathrm{H}, \mathrm{m}), 0.95-$ $1.00(2 \mathrm{H}, \mathrm{m}), 2.00-2.10(1 \mathrm{H}, \mathrm{m}), 2.95-3.00(1 \mathrm{H}, \mathrm{m}), 3.45-3.50(1 \mathrm{H}, \mathrm{m})$, $6.81-6.87(1 \mathrm{H}, \mathrm{m}), 6.89-6.95(1 \mathrm{H}, \mathrm{m}), 7.50-7.56(1 \mathrm{H}, \mathrm{m}) .{ }^{19} \mathrm{~F}-\mathrm{NMR}$ $\left(\mathrm{CDCl}_{3}\right) \delta:-7.2(1 \mathrm{~F}, \mathrm{dd}, J=12,214 \mathrm{~Hz}),-8.0(1 \mathrm{~F}, \mathrm{dd}, J=10,214 \mathrm{~Hz})$, $-31.5-31.6(1 \mathrm{~F}, \mathrm{~m}),-33.1--33.2(1 \mathrm{~F}, \mathrm{~m})$. MS $m / z(\%): 278\left(2, \mathrm{M}^{+}\right)$, 127 (100). HRMS m/z: 278.0392 (Calcd for $\mathrm{C}_{12} \mathrm{H}_{10} \mathrm{~F}_{4} \mathrm{OS}$ : 278.0389). IR (neat) $\mathrm{cm}^{-1}: 1230$.

2-[(Butylthio)difluoromethyl]-2-(2,4-difluorophenyl)oxirane (8e): Colorless oil. Yield $61 \%$. ${ }^{1} \mathrm{H}-\mathrm{NMR}\left(\mathrm{CDCl}_{3}\right) \delta: 0.90(3 \mathrm{H}, \mathrm{t}, J=7.3 \mathrm{~Hz}), 1.36-1.43$ $(2 \mathrm{H}, \mathrm{m}), 1.57-1.65(2 \mathrm{H}, \mathrm{m}), 2.83(2 \mathrm{H}, \mathrm{dt}, J=2.0,7.3 \mathrm{~Hz}), 2.85-3.00(1 \mathrm{H}$, $\mathrm{m}), 3.48-3.50(1 \mathrm{H}, \mathrm{m}) .6 .80-6.87(1 \mathrm{H}, \mathrm{m}), 6.90-6.95(1 \mathrm{H}, \mathrm{m}), 7.50-$ $7.57(1 \mathrm{H}, \mathrm{m}) .{ }^{19} \mathrm{~F}-\mathrm{NMR}\left(\mathrm{CDCl}_{3}\right) \delta:-5.6(1 \mathrm{~F}, \mathrm{dd}, J=10,214 \mathrm{~Hz}),-6.9(1 \mathrm{~F}$, dd, $J=14,214 \mathrm{~Hz}),-31.5--31.6(1 \mathrm{~F}, \mathrm{~m}),-33.2--33.3(1 \mathrm{~F}, \mathrm{~m})$. MS $\mathrm{m} / \mathrm{z}$ (\%): $294\left(12, \mathrm{M}^{+}\right), 155(100)$. HRMS $m / z$ : 294.0688 (Calcd for $\mathrm{C}_{13} \mathrm{H}_{14} \mathrm{~F}_{4} \mathrm{OS}$ : 294.0702). IR (neat) $\mathrm{cm}^{-1}: 1235$.

2-[(tert-Butylthio)difluoromethyl]-2-(2,4-difluorophenyl)oxirane (8f): Colorless oil. Yield 98\%. ${ }^{1} \mathrm{H}-\mathrm{NMR}\left(\mathrm{CDCl}_{3}\right) \delta: 1.46(9 \mathrm{H}, \mathrm{s}), 2.94-2.97$ $(1 \mathrm{H}, \mathrm{m}), 3.38-3.40(1 \mathrm{H}, \mathrm{m}), 6.80-6.94(2 \mathrm{H}, \mathrm{m}), 7.50-7.57(1 \mathrm{H}, \mathrm{m}) .{ }^{19} \mathrm{~F}-$ NMR $\left(\mathrm{CDCl}_{3}\right) \delta:-4.18(1 \mathrm{~F}, \mathrm{dd}, J=11,211 \mathrm{~Hz}),-4.57(1 \mathrm{~F}, \mathrm{dd}, J=7$, $211 \mathrm{~Hz}),-31.7-31.8(1 \mathrm{~F}, \mathrm{~m}),-33.0-33.1(1 \mathrm{~F}, \mathrm{~m}) . \mathrm{MS} m / z: 294(2$, $\mathrm{M}^{+}$), 57 (100). HRMS $m / z$ : 294.0704 (Calcd for $\left.\mathrm{C}_{13} \mathrm{H}_{14} \mathrm{~F}_{4} \mathrm{OS}: 294.0701\right)$. IR (neat) $\mathrm{cm}^{-1}: 1270$.

2-[Difluoro(pentylthio)methyl]-2-(2,4-difluorophenyl)oxirane (8g): Colorless oil. Yield $67 \% .{ }^{1} \mathrm{H}-\mathrm{NMR}\left(\mathrm{CDCl}_{3}\right) \delta: 0.90(3 \mathrm{H}, \mathrm{t}, J=7.3 \mathrm{~Hz}), 1.35-1.95$ $(6 \mathrm{H}, \mathrm{m}), 2.83(2 \mathrm{H}, \mathrm{dt}, J=2.0,7.3 \mathrm{~Hz}), 2.85-3.00(1 \mathrm{H}, \mathrm{m}), 3.48-3.50(1 \mathrm{H}$, m). $6.80-6.87(1 \mathrm{H}, \mathrm{m}), 6.90-6.95(1 \mathrm{H}, \mathrm{m}), 7.50-7.57(1 \mathrm{H}, \mathrm{m}) .{ }^{19} \mathrm{~F}-\mathrm{NMR}$ $\left(\mathrm{CDCl}_{3}\right) \delta:-5.5(1 \mathrm{~F}, \mathrm{dd}, J=10,214 \mathrm{~Hz}),-6.9(1 \mathrm{~F}, \mathrm{dd}, J=14,214 \mathrm{~Hz})$, $-31.5-31.6(1 \mathrm{~F}, \mathrm{~m}),-33.2-33.3(1 \mathrm{~F}, \mathrm{~m})$. MS $m / z(\%): 308\left(9, \mathrm{M}^{+}\right)$, 155 (100). HRMS m/z: 308.0861 (Calcd for $\mathrm{C}_{14} \mathrm{H}_{16} \mathrm{~F}_{4} \mathrm{OS}: 308.0858$ ). IR (neat) $\mathrm{cm}^{-1}: 1235$.

2-[Difluoro(methylthio)methyl]-2-phenyloxirane (8h): Colorless oil. Yield 95\%. ${ }^{1} \mathrm{H}-\mathrm{NMR}\left(\mathrm{CDCl}_{3}\right) \delta: 2.26(3 \mathrm{H}, \mathrm{s}), 2.85-2.90(1 \mathrm{H}, \mathrm{m}), 3.45-3.47$ $(1 \mathrm{H}, \mathrm{m}), 7.36-7.40(3 \mathrm{H}, \mathrm{m}), 7.53-7.56(2 \mathrm{H}, \mathrm{m}) .{ }^{19} \mathrm{~F}-\mathrm{NMR}\left(\mathrm{CDCl}_{3}\right) \delta$ : $-0.4(1 \mathrm{~F}, \mathrm{~d}, J=215 \mathrm{~Hz}),-4.2(1 \mathrm{~F}, \mathrm{~d}, J=215 \mathrm{~Hz})$. MS $m / z(\%): 216(18$, $\mathrm{M}^{+}$), 133 (100). HRMS $m / z$ : 216.0401 (Calcd for $\mathrm{C}_{10} \mathrm{H}_{10} \mathrm{~F}_{2} \mathrm{OS}: 216.0420$ ). IR (neat) $\mathrm{cm}^{-1}: 1240$.

2-[Difluoro(methylthio)methyl]-2-(4-fluorophenyl)oxirane (8i): Colorless oil. Yield 97\%. ${ }^{1} \mathrm{H}-\mathrm{NMR}\left(\mathrm{CDCl}_{3}\right) \delta: 2.27(3 \mathrm{H}, \mathrm{s}), 2.85-2.90(1 \mathrm{H}, \mathrm{m})$, $3.45-3.50(1 \mathrm{H}, \mathrm{m}), 7.05-7.10(2 \mathrm{H}, \mathrm{m}), 7.50-7.55(2 \mathrm{H}, \mathrm{m}) .{ }^{19} \mathrm{~F}-\mathrm{NMR}$ $\left(\mathrm{CDCl}_{3}\right) \delta$ : $-5.8(1 \mathrm{~F}, \mathrm{dd}, J=6,214 \mathrm{~Hz}),-6.3(1 \mathrm{~F}, \mathrm{dd}, J=10,214 \mathrm{~Hz}),-36.1$ (1F, s). MS $m / z(\%): 234\left(3, \mathrm{M}^{+}\right), 61$ (100). HRMS $m / z$ : 234.0311 (Calcd for $\left.\mathrm{C}_{10} \mathrm{H}_{9} \mathrm{~F}_{3} \mathrm{OS}: 220.0170\right)$. IR (neat) $\mathrm{cm}^{-1}: 1225$.

2-[Difluoro(methylthio)methyl]-2-[4-(trifluoromethyl)phenyl]oxirane (8j): Colorless oil. Yield 99\%. ${ }^{1} \mathrm{H}-\mathrm{NMR}\left(\mathrm{CDCl}_{3}\right) \delta: 2.29(3 \mathrm{H}, \mathrm{s}), 2.85-2.90$ $(1 \mathrm{H}, \mathrm{m}), 3.50-3.52(1 \mathrm{H}, \mathrm{m}), 7.65(2 \mathrm{H}, \mathrm{d}, J=6.1 \mathrm{~Hz}), 7.67(2 \mathrm{H}, \mathrm{d}$, $J=6.1 \mathrm{~Hz}) .{ }^{19} \mathrm{~F}-\mathrm{NMR}\left(\mathrm{CDCl}_{3}\right) \delta: 12.9(3 \mathrm{~F}, \mathrm{~s}),-4.9(1 \mathrm{~F}, \mathrm{~d}, J=216 \mathrm{~Hz}),-9.1$ (1F, d, $J=216 \mathrm{~Hz})$. MS $m / z(\%): 284\left(28, \mathrm{M}^{+}\right), 159$ (100). HRMS $m / z$ : 284.0291 (Calcd for $\mathrm{C}_{11} \mathrm{H}_{9} \mathrm{~F}_{5} \mathrm{OS}: 284.0294$ ). IR (neat) $\mathrm{cm}^{-1}: 1240$.

2-(2,4-Dichlorophenyl)-2-[difluoro(methylthio)methyl]oxirane (8k): Colorless oil. Yield 83\%. ${ }^{1} \mathrm{H}-\mathrm{NMR}\left(\mathrm{CDCl}_{3}\right) \delta: 2.29(3 \mathrm{H}, \mathrm{s}), 3.03-3.05(1 \mathrm{H}$, $\mathrm{m}), 3.58-3.60(1 \mathrm{H}, \mathrm{m}), 7.30(1 \mathrm{H}, \mathrm{dd}, J=2.0,8.8 \mathrm{~Hz}), 7.42(1 \mathrm{H}, \mathrm{d}$, $J=2.0 \mathrm{~Hz}), 7.54(1 \mathrm{H}, \mathrm{d}, J=8.8 \mathrm{~Hz}) \cdot{ }^{19} \mathrm{~F}-\mathrm{NMR}\left(\mathrm{CDCl}_{3}\right) \delta:-6.7(1 \mathrm{~F}, \mathrm{~d}$, $J=255 \mathrm{~Hz}),-7.5(1 \mathrm{~F}, \mathrm{~d}, J=255 \mathrm{~Hz})$. MS $m / z(\%): 284\left(6, \mathrm{M}^{+}\right), 249(100)$. 
HRMS $m / z$ : 283.9677 (Calcd for $\mathrm{C}_{10} \mathrm{H}_{8} \mathrm{Cl}_{2} \mathrm{~F}_{2} \mathrm{OS}: 216.0420$ ). IR (neat) $\mathrm{cm}^{-1}$ : 1245 .

2-[[[2-(2,4-Difluorophenyl)-2-oxiranyl]difluoromethyl]thio]ethyl acetate (81): Colorless oil. Yield 90\%. ${ }^{1} \mathrm{H}-\mathrm{NMR}\left(\mathrm{CDCl}_{3}\right) \delta: 2.06(3 \mathrm{H}, \mathrm{s}), 2.95-3.00$ $(1 \mathrm{H}, \mathrm{m}), 3.07(2 \mathrm{H}, \mathrm{dd}, J=1.0,6.8 \mathrm{~Hz}), 3.48-3.51(1 \mathrm{H}, \mathrm{m}), 4.24(2 \mathrm{H}, \mathrm{t}$, $J=6.4 \mathrm{~Hz}), 6.82-6.88(1 \mathrm{H}, \mathrm{m}), 6.90-6.96(1 \mathrm{H}, \mathrm{m}), 7.50-7.56(1 \mathrm{H}, \mathrm{m})$. ${ }^{19} \mathrm{~F}-\mathrm{NMR}\left(\mathrm{CDCl}_{3}\right) \delta:-4.3(1 \mathrm{~F}, \mathrm{dd}, J=10,211 \mathrm{~Hz}),-5.9(1 \mathrm{~F}, \mathrm{dd}, J=5$, $211 \mathrm{~Hz}),-31.1--31.2(1 \mathrm{~F}, \mathrm{~m}),-33.1--33.2(1 \mathrm{~F}, \mathrm{~m})$. MS $m / z(\%): 324$ $\left(0.2, \mathrm{M}^{+}\right.$), 205 (100). HRMS $m / z: 324.0450$ (Calcd for $\mathrm{C}_{13} \mathrm{H}_{12} \mathrm{~F}_{4} \mathrm{O}_{3} \mathrm{~S}$ : 324.0444). IR (neat) $\mathrm{cm}^{-1}: 1745,1230$.

2-(2,4-Difluorophenyl)-2-[difluoro(phenylthio)methyl]oxirane (8n): Colorless oil. Yield 49\%. ${ }^{1} \mathrm{H}-\mathrm{NMR}\left(\mathrm{CDCl}_{3}\right) \delta: 3.00-3.03(1 \mathrm{H}, \mathrm{m}), 3.50-3.52$ $(1 \mathrm{H}, \mathrm{m}), 6.83-6.86(1 \mathrm{H}, \mathrm{m}), 6.87-7.00(1 \mathrm{H}, \mathrm{m}), 7.32-7.38(3 \mathrm{H}, \mathrm{m})$, $7.39-7.44(1 \mathrm{H}, \mathrm{m}), 7.48-7.61(2 \mathrm{H}, \mathrm{m}) .{ }^{19} \mathrm{~F}-\mathrm{NMR}\left(\mathrm{CDCl}_{3}\right) \delta:-5.1(1 \mathrm{~F}$, $\mathrm{dd}, J=10,213 \mathrm{~Hz}),-6.3(1 \mathrm{~F}, \mathrm{dd}, J=7,213 \mathrm{~Hz}),-31.3--31.4(1 \mathrm{~F}, \mathrm{~m})$, $-33.0-33.2(1 \mathrm{~F}, \mathrm{~m})$. MS m/z: $314\left(0.2, \mathrm{M}^{+}\right), 91$ (100). HRMS $m / z$ : 314.0364 (Calcd for $\mathrm{C}_{15} \mathrm{H}_{10} \mathrm{~F}_{4} \mathrm{OS}: 314.0388$ ). IR (neat) $\mathrm{cm}^{-1}: 1270$.

2-[Difluoro[(4-methylphenyl)thio]methyl]-2-(2,4-difluorophenyl)oxirane (8o): Colorless oil. Yield 52\%. ${ }^{1} \mathrm{H}-\mathrm{NMR}\left(\mathrm{CDCl}_{3}\right) \delta: 2.35(3 \mathrm{H}, \mathrm{s}), 3.00-$ $3.05(1 \mathrm{H}, \mathrm{m}), 3.45-3.50(1 \mathrm{H}, \mathrm{m}), 6.82-6.88(1 \mathrm{H}, \mathrm{m}), 6.90-6.96(1 \mathrm{H}, \mathrm{m})$, $7.16(2 \mathrm{H}, \mathrm{d}, J=8.3 \mathrm{~Hz}), 7.44(2 \mathrm{H}, \mathrm{d}, J=8.3 \mathrm{~Hz}), 7.54-7.60(1 \mathrm{H}, \mathrm{m}) .{ }^{19} \mathrm{~F}-$ $\operatorname{NMR}\left(\mathrm{CDCl}_{3}\right) \delta:-4.5(1 \mathrm{~F}, \mathrm{dd}, J=10,209 \mathrm{~Hz}),-5.5(1 \mathrm{~F}, \mathrm{dd}, J=7,210 \mathrm{~Hz})$, $-31.3--31.4(1 \mathrm{~F}, \mathrm{~m}),-32.9-33.0(1 \mathrm{~F}, \mathrm{~m})$. MS m/z (\%): $328\left(74, \mathrm{M}^{+}\right)$, 169 (100). HRMS $m / z$ : 328.0533 (Calcd for $\mathrm{C}_{16} \mathrm{H}_{12} \mathrm{~F}_{4} \mathrm{OS}: 328.0545$ ). IR (neat) $\mathrm{cm}^{-1}: 1270$

2-[(Benzylthio)difluoromethyl]-2-(2,4-difluorophenyl)oxirane (8p): Colorless oil. Yield 99\%. ${ }^{1} \mathrm{H}-\mathrm{NMR}\left(\mathrm{CDCl}_{3}\right) \delta: 2.97-3.00(1 \mathrm{H}, \mathrm{m}), 3.49-3.50$ $(1 \mathrm{H}, \mathrm{m}), 4.07(2 \mathrm{H}, \mathrm{s}), 6.80-6.85(1 \mathrm{H}, \mathrm{m}), 6.86-6.92(1 \mathrm{H}, \mathrm{m}), 7.26-7.34$ $(5 \mathrm{H}, \mathrm{m}), 7.50-7.55(1 \mathrm{H}, \mathrm{m}) .{ }^{19} \mathrm{~F}-\mathrm{NMR}\left(\mathrm{CDCl}_{3}\right) \delta:-5.2(1 \mathrm{~F}, \mathrm{dd}, J=10$, $213 \mathrm{~Hz}),-6.3(1 \mathrm{~F}, \mathrm{dd}, J=10,213 \mathrm{~Hz}),-31.2--31.3(1 \mathrm{~F}, \mathrm{~m}),-33.0-$ $-33.1(1 \mathrm{~F}, \mathrm{~m})$. MS $m / z$ : $328\left(0.3, \mathrm{M}^{+}\right), 91(100)$. HRMS $m / z: 328.0530$ (Calcd for $\mathrm{C}_{16} \mathrm{H}_{12} \mathrm{~F}_{4} \mathrm{OS}$ : 328.0545). IR (neat) $\mathrm{cm}^{-1}: 1270$.

2-[Difluoro[(4-methoxybenzyl)thio]methyl]-2-(2,4-difluorophenyl)oxirane (8q): Colorless oil. Yield 71\%. ${ }^{1} \mathrm{H}-\mathrm{NMR}\left(\mathrm{CDCl}_{3}\right) \delta: 2.97-3.00(1 \mathrm{H}$, $\mathrm{m}), 3.48-3.50(1 \mathrm{H}, \mathrm{m}), 3.78(3 \mathrm{H}, \mathrm{s}), 4.04(2 \mathrm{H}, \mathrm{s}), 6.80-6.85(1 \mathrm{H}, \mathrm{m}), 6.83$ $(2 \mathrm{H}, \mathrm{d}, J=8.8 \mathrm{~Hz}), 6.86-6.93(1 \mathrm{H}, \mathrm{m}), 7.22(2 \mathrm{H}, \mathrm{d}, J=8.8 \mathrm{~Hz}), 7.45-7.55$ $(1 \mathrm{H}, \mathrm{m}) .{ }^{19} \mathrm{~F}-\mathrm{NMR}\left(\mathrm{CDCl}_{3}\right) \delta:-5.3(1 \mathrm{~F}, \mathrm{dd}, J=10,214 \mathrm{~Hz}),-6.5(1 \mathrm{~F}, \mathrm{dd}$, $J=5,214 \mathrm{~Hz}),-31.3--31.4(1 \mathrm{~F}, \mathrm{~m}),-33.1-33.2(1 \mathrm{~F}, \mathrm{~m})$. MS $m / z(\%)$ : $358\left(34, \mathrm{M}^{+}\right), 121(100)$. HRMS $m / z$ : 358.0681 (Calcd for $\mathrm{C}_{17} \mathrm{H}_{14} \mathrm{~F}_{4} \mathrm{O}_{2} \mathrm{~S}$ : 358.0650). IR (neat) $\mathrm{cm}^{-1}: 1250$.

General Procedure for the Synthesis of 1,1-Difluoro-1-(substituted thio)-3-(1H-1,2,4-triazol-1-yl)-2-propanol (9) 1,2,4-Triazole (2.2 mmol) and $\mathrm{K}_{2} \mathrm{CO}_{3}(1.1 \mathrm{mmol})$ were added to a solution of $\mathbf{8}(2 \mathrm{mmol})$ in dimethylformamide (DMF) $(30 \mathrm{ml})$. The mixture was warmed at $65^{\circ} \mathrm{C}$ for $5 \mathrm{~h}$. After addition of aqueous $\mathrm{NaHCO}_{3}$, the mixture was extracted with AcOEt. The organic extract was washed with water and brine, dried over $\mathrm{MgSO}_{4}$, and evaporated under reduced pressure. The residue was chromatographed on a silica gel column using $\mathrm{CHCl}_{3}-\mathrm{MeOH}[98: 2(\mathrm{v} / \mathrm{v})]$ as a mobile phase to give 9.

Resolution of ( \pm )-9 into ( +$)-9$ and (-)-9 Compound $( \pm)-9(50 \mathrm{mg})$ was subjected to preparative HPLC (CHIRALCEL OD with pre-column CHIRALCEL OD), using $n$-hexane-2-propanol $[3: 1(\mathrm{v} / \mathrm{v})]$ as a mobile phase. The more mobile isomer is $(-)-9$ and the less mobile isomer is $(+)-$ 9. Their optical yields are measured by HPLC (CHIRALCEL OD with precolumn CHIRALCEL OD) using $n$-hexane-2-propanol $[1: 1(\mathrm{v} / \mathrm{v})]$ as a mobile phase (flow rate: $0.5 \mathrm{ml} / \mathrm{min}$, column temperature: $21^{\circ} \mathrm{C}$ ).

2-(2,4-Difluorophenyl)-1,1-difluoro-1-(methylthio)-3-(1H-1,2,4-triazol-1yl)-2-propanol (9a): Colorless powder. $\mathrm{mp} 122.2^{\circ} \mathrm{C}$. Yield $72 \%$. ${ }^{1} \mathrm{H}-\mathrm{NMR}$ $\left(\mathrm{CDCl}_{3}\right) \delta: 2.27(3 \mathrm{H}, \mathrm{s}), 4.82(1 \mathrm{H}, \mathrm{d}, J=14.2 \mathrm{~Hz}), 5.28(1 \mathrm{H}, \mathrm{d}, J=14.2 \mathrm{~Hz})$, $5.76(1 \mathrm{H}, \mathrm{s}), 6.71-6.75(1 \mathrm{H}, \mathrm{m}), 6.76-6.88(1 \mathrm{H}, \mathrm{m}), 7.71-7.77(1 \mathrm{H}, \mathrm{m})$, $7.80(1 \mathrm{H}, \mathrm{s}), 8.09(1 \mathrm{H}, \mathrm{s}) .{ }^{19} \mathrm{~F}-\mathrm{NMR}\left(\mathrm{CDCl}_{3}\right) \delta:-9.4(1 \mathrm{~F}, \mathrm{dd}, J=25$, $211 \mathrm{~Hz}),-11.8(1 \mathrm{~F}, \mathrm{dd}, J=15,211 \mathrm{~Hz}),-30.4-30.5(1 \mathrm{~F}, \mathrm{~m}),-32.2-$ -32.3 (1F, m). MS m/z (\%): $321\left(1, \mathrm{M}^{+}\right), 224$ (100). HRMS $m / z: 321.0546$ (Calcd for $\left.\mathrm{C}_{12} \mathrm{H}_{11} \mathrm{~F}_{4} \mathrm{~N}_{3} \mathrm{OS}: 321.0559\right)$. IR (KBr) $\mathrm{cm}^{-1}: 3100$.

$(+)-9 \mathrm{a}$ : Colorless powder, mp $154-156^{\circ} \mathrm{C}$, e.e. $99.2 \%,[\alpha]_{\mathrm{D}}^{25}: 64.6^{\circ}$ $(c=0.5$, acetone), retention time $(\mathrm{min}): 9.23$.

(-)-9a: Colorless powder, mp $154-156^{\circ} \mathrm{C}$, e.e. $99.2 \%,[\alpha]_{\mathrm{D}}^{25}:-65.0^{\circ}$ $(c=0.5$, acetone), retention time $(\mathrm{min}): 8.80$.

2-(2,4-Difluorophenyl)-1-(ethylthio)-1,1-difluoro-3-(1H-1,2,4-triazol-1yl)-2-propanol (9b): Colorless powder. mp $110.7^{\circ} \mathrm{C}$. Yield $85 \%$. ${ }^{1} \mathrm{H}-\mathrm{NMR}$ $\left(\mathrm{CDCl}_{3}\right) \delta: 1.30(3 \mathrm{H}, \mathrm{t}, J=7.3 \mathrm{~Hz}), 2.85(2 \mathrm{H}, \mathrm{q}, J=7.3 \mathrm{~Hz}), 4.82(1 \mathrm{H}, \mathrm{d}$, $J=14.2 \mathrm{~Hz}), 5.28(1 \mathrm{H}, \mathrm{d}, J=14.2 \mathrm{~Hz}), 5.71(1 \mathrm{H}, \mathrm{s}), 6.71-6.77(1 \mathrm{H}, \mathrm{m})$, $6.82-6.88(1 \mathrm{H}, \mathrm{m}), 7.70-7.77(1 \mathrm{H}, \mathrm{m}), 7.81(1 \mathrm{H}, \mathrm{s}), 8.09(1 \mathrm{H}, \mathrm{s}) .{ }^{19} \mathrm{~F}-$ NMR $\left(\mathrm{CDCl}_{3}\right) \delta:-5.9(1 \mathrm{~F}, \mathrm{dd}, J=25,214 \mathrm{~Hz}),-9.3(1 \mathrm{~F}, \mathrm{dd}, J=12$,
$214 \mathrm{~Hz}),-30.5--30.6(1 \mathrm{~F}, \mathrm{~m}),-32.3--32.4(1 \mathrm{~F}, \mathrm{~m}) . \mathrm{MS} m / z(\%): 336$ $\left(4, \mathrm{M}^{+}+1\right), 224$ (100). HRMS $m / z: 336.0820$ (Calcd for $\mathrm{C}_{13} \mathrm{H}_{14} \mathrm{~F}_{4} \mathrm{~N}_{3} \mathrm{OS}$ : 336.0794). IR (KBr) cm $\mathrm{cm}^{-1}$ : 3100. Anal. Calcd for $\mathrm{C}_{13} \mathrm{H}_{13} \mathrm{~F}_{4} \mathrm{~N}_{3} \mathrm{OS}: \mathrm{C}$, 46.56; H, 3.91; N, 12.53. Found: C, 46.15; H, 3.87; N, 12.43.

2-(2,4-Difluorophenyl)-1,1-difluoro-1-propylthio-3-(1H-1,2,4-triazol-1yl)-2-propanol (9c): Colorless powder. mp $86.7^{\circ} \mathrm{C}$. Yield $32 \%$. ${ }^{1} \mathrm{H}-\mathrm{NMR}$ $\left(\mathrm{CDCl}_{3}\right) \delta$ : $0.97(3 \mathrm{H}, \mathrm{t}, J=7.3 \mathrm{~Hz}), 1.61-1.70(2 \mathrm{H}, \mathrm{m}), 2.80(2 \mathrm{H}, \mathrm{t}$, $J=7.3 \mathrm{~Hz}), 4.82(1 \mathrm{H}, \mathrm{d}, J=14.2 \mathrm{~Hz}), 5.28(1 \mathrm{H}, \mathrm{d}, J=14.2 \mathrm{~Hz}), 5.65(1 \mathrm{H}, \mathrm{s})$, $6.70-6.77(1 \mathrm{H}, \mathrm{m}), 6.83-6.88(1 \mathrm{H}, \mathrm{m}), 7.70-7.77(1 \mathrm{H}, \mathrm{m}), 7.82(1 \mathrm{H}, \mathrm{s})$, $8.09(1 \mathrm{H}, \mathrm{s}) .{ }^{19} \mathrm{~F}-\mathrm{NMR}\left(\mathrm{CDCl}_{3}\right) \delta:-5.4(1 \mathrm{~F}, \mathrm{dd}, J=25,211 \mathrm{~Hz}),-8.7(1 \mathrm{~F}$, $\mathrm{dd}, J=16,211 \mathrm{~Hz}),-30.2--30.3(1 \mathrm{~F}, \mathrm{~m}),-32.0--32.1(1 \mathrm{~F}, \mathrm{~m}) . \mathrm{MS} m / z$ (\%): $349\left(1, \mathrm{M}^{+}\right), 224$ (100). HRMS m/z: 349.0887 (Calcd for $\mathrm{C}_{14} \mathrm{H}_{15} \mathrm{~F}_{4} \mathrm{~N}_{3} \mathrm{OS}$ : 349.0871). IR $(\mathrm{KBr}) \mathrm{cm}^{-1}$ : 3100. Anal. Calcd for $\mathrm{C}_{14} \mathrm{H}_{15} \mathrm{~F}_{4} \mathrm{~N}_{3} \mathrm{OS}$ : C, 48.13; H, 4.33; N, 12.03. Found: C, 8.41; H, 4.34; N, 12.07 .

1-(Cyclopropylthio)-2-(2,4-difluorophenyl)-1,1-difluoro-3-(1H-1,2,4-triazol-1-yl)-2-propanol (9d): Colorless powder. mp $102.1^{\circ} \mathrm{C}$. Yield $57 \%$. ${ }^{1} \mathrm{H}-$ $\operatorname{NMR}\left(\mathrm{CDCl}_{3}\right) \delta: 0.65-0.70(2 \mathrm{H}, \mathrm{m}), 0.95-1.00(2 \mathrm{H}, \mathrm{m}), 2.00-2.05(1 \mathrm{H}$, $\mathrm{m}), 4.81(1 \mathrm{H}, \mathrm{d}, J=14.2 \mathrm{~Hz}), 5.28(1 \mathrm{H}, \mathrm{d}, J=14.2 \mathrm{~Hz}), 5.68(1 \mathrm{H}, \mathrm{s}), 6.70-$ $6.75(1 \mathrm{H}, \mathrm{m}), 6.80-6.85(1 \mathrm{H}, \mathrm{m}), 7.70-7.75(1 \mathrm{H}, \mathrm{m}), 7.81(1 \mathrm{H}, \mathrm{s}), 8.09$ $(1 \mathrm{H}, \mathrm{s}) .{ }^{19} \mathrm{~F}-\mathrm{NMR}\left(\mathrm{CDCl}_{3}\right) \delta:-7.6(1 \mathrm{~F}, \mathrm{dd}, J=24,214 \mathrm{~Hz}),-10.1(1 \mathrm{~F}, \mathrm{dd}$, $J=22,214 \mathrm{~Hz}),-30.6-30.7(1 \mathrm{~F}, \mathrm{~m}),-32.3--32.4(1 \mathrm{~F}, \mathrm{~m})$. MS $\mathrm{m} / \mathrm{z}$ (\%): $347\left(4, \mathrm{M}^{+}\right), 224$ (100). HRMS $m / z: 347.0710$ (Calcd for $\mathrm{C}_{14} \mathrm{H}_{13} \mathrm{~F}_{4} \mathrm{~N}_{3} \mathrm{OS}$ : 347.0715). IR (KBr) $\mathrm{cm}^{-1}$ : 3100. Anal. Calcd for $\mathrm{C}_{14} \mathrm{H}_{13} \mathrm{~F}_{4} \mathrm{~N}_{3} \mathrm{OS}$ : C, 48.41; H, 3.77; N, 12.10; S, 9.23. Found: C, 48.62; H, $3.82 ; \mathrm{N}, 12.09 ; \mathrm{S}, 9.14$.

1-(Butylthio)-2-(2,4-difluorophenyl)-1,1-difluoro-3-(1H-1,2,4-triazol-1yl)-2-propanol (9e): Colorless powder. mp $77.1^{\circ} \mathrm{C}$. Yield $61 \%$. ${ }^{1} \mathrm{H}-\mathrm{NMR}$ $\left(\mathrm{CDCl}_{3}\right) \delta$ : $0.90(3 \mathrm{H}, \mathrm{t}, J=7.3 \mathrm{~Hz}), 1.36-1.41(2 \mathrm{H}, \mathrm{m}), 1.57-1.64(2 \mathrm{H}$, $\mathrm{m}), 2.82(2 \mathrm{H}, \mathrm{t}, J=7.3 \mathrm{~Hz}), 4.82(1 \mathrm{H}, \mathrm{d}, J=14.6 \mathrm{~Hz}), 5.28(1 \mathrm{H}, \mathrm{d}$, $J=14.6 \mathrm{~Hz}), 5.68(1 \mathrm{H}, \mathrm{s}), 6.71-6.77(1 \mathrm{H}, \mathrm{m}), 6.82-6.88(1 \mathrm{H}, \mathrm{m}), 7.70-$ $7.77(1 \mathrm{H}, \mathrm{m}), 7.81(1 \mathrm{H}, \mathrm{s}), 8.09(1 \mathrm{H}, \mathrm{s}) .{ }^{19} \mathrm{~F}-\mathrm{NMR}\left(\mathrm{CDCl}_{3}\right) \delta:-5.8(1 \mathrm{~F}, \mathrm{dd}$, $J=25,211 \mathrm{~Hz}),-9.0(1 \mathrm{~F}, \mathrm{dd}, J=16,211 \mathrm{~Hz}),-30.4-30.6(1 \mathrm{~F}, \mathrm{~m})$, $-32.3--32.4(1 \mathrm{~F}, \mathrm{~m})$. MS $m / z(\%): 364\left(1, \mathrm{M}^{+}+1\right), 224$ (100). HRMS $m / z: 364.1014$ (Calcd for $\mathrm{C}_{15} \mathrm{H}_{18} \mathrm{~F}_{4} \mathrm{~N}_{3} \mathrm{OS}: 349.0871$ ). IR (KBr) cm $\mathrm{cm}^{-1}: 3100$. Anal. Calcd for $\mathrm{C}_{15} \mathrm{H}_{17} \mathrm{~F}_{4} \mathrm{~N}_{3} \mathrm{OS}$ : C, 49.58; H, 4.72; N, 11.56; S, 8.82. Found: C, 49.68; H, 4.77; N, 11.54; S, 8.82.

1-(tert-Butylthio)-2-(2,4-difluorophenyl)-1,1-difluoro-3-(1H-1,2,4-triazol1-yl)-2-propanol (9f): Colorless powder. mp $142.7^{\circ} \mathrm{C}$. Yield 42\%. ${ }^{1} \mathrm{H}-\mathrm{NMR}$ $\left(\mathrm{CDCl}_{3}\right) \delta: 1.46(9 \mathrm{H}, \mathrm{s}), 5.28(1 \mathrm{H}, \mathrm{d}, J=14.7 \mathrm{~Hz}), 5.28(1 \mathrm{H}, \mathrm{d}, J=14.7 \mathrm{~Hz})$, $5.78(1 \mathrm{H}, \mathrm{s}), 6.70-6.78(1 \mathrm{H}, \mathrm{m}), 6.81-6.86(1 \mathrm{H}, \mathrm{m}), 7.68-7.74(1 \mathrm{H}, \mathrm{m})$, $7.77(1 \mathrm{H}, \mathrm{s}), 8.09(1 \mathrm{H}, \mathrm{s}) .{ }^{19} \mathrm{~F}-\mathrm{NMR}\left(\mathrm{CDCl}_{3}\right) \delta:-1.9(1 \mathrm{~F}, \mathrm{dd}, J=25$, $211 \mathrm{~Hz}),-6.7(1 \mathrm{~F}, \mathrm{dd}, J=17,211 \mathrm{~Hz}),-30.1--30.2(1 \mathrm{~F}, \mathrm{~m}),-32.6$ $-32.7(1 \mathrm{~F}, \mathrm{~m})$. MS $m / z(\%): 363\left(0.8, \mathrm{M}^{+}\right), 224(100)$. HRMS $m / z$ : 363.1020 (Calcd for $\left.\mathrm{C}_{15} \mathrm{H}_{17} \mathrm{~F}_{4} \mathrm{~N}_{3} \mathrm{OS}: 363.1028\right)$. IR (KBr) $\mathrm{cm}^{-1}$ : 3100. Anal. Calcd for $\mathrm{C}_{15} \mathrm{H}_{17} \mathrm{~F}_{4} \mathrm{~N}_{3} \mathrm{OS}$ : C, 49.58; H, 4.72; N, 11.56. Found: C, 49.80; H, $4.80 ; \mathrm{N}, 11.70$.

2-(2,4-Difluorophenyl)-1,1-difluoro-1-(pentylthio)-3-(1H-1,2,4-triazol-1yl)-2-propanol $(\mathbf{9 g})$ : Colorless powder. mp $59{ }^{\circ} \mathrm{C}$. Yield $58 \%$. ${ }^{1} \mathrm{H}-\mathrm{NMR}$ $\left(\mathrm{CDCl}_{3}\right) \delta: 0.90(3 \mathrm{H}, \mathrm{t}, J=7.3 \mathrm{~Hz}), 1.17-1.80(6 \mathrm{H}, \mathrm{m}), 2.82(2 \mathrm{H}, \mathrm{t}$, $J=7.3 \mathrm{~Hz}), 4.82(1 \mathrm{H}, \mathrm{d}, J=14.6 \mathrm{~Hz}), 5.28(1 \mathrm{H}, \mathrm{d}, J=14.6 \mathrm{~Hz}), 5.68(1 \mathrm{H}, \mathrm{s})$, $6.71-6.77(1 \mathrm{H}, \mathrm{m}), 6.82-6.88(1 \mathrm{H}, \mathrm{m}), 7.70-7.77(1 \mathrm{H}, \mathrm{m}), 7.81(1 \mathrm{H}, \mathrm{s})$, $8.09(1 \mathrm{H}, \mathrm{s}) .{ }^{19} \mathrm{~F}-\mathrm{NMR}\left(\mathrm{CDCl}_{3}\right) \delta:-5.8(1 \mathrm{~F}, \mathrm{dd}, J=25,211 \mathrm{~Hz}),-9.0(1 \mathrm{~F}$, $\mathrm{dd}, J=16,211 \mathrm{~Hz}),-30.4-30.6(1 \mathrm{~F}, \mathrm{~m}),-32.3--32.4(1 \mathrm{~F}, \mathrm{~m}) . \mathrm{MS} m / z$ (\%): $378\left(1, \mathrm{M}^{+}+1\right), 224$ (100). HRMS m/z: 378.1242 (Calcd for $\mathrm{C}_{16} \mathrm{H}_{20} \mathrm{~F}_{4} \mathrm{~N}_{3} \mathrm{OS}:$ 378.1263). IR $(\mathrm{KBr}) \mathrm{cm}^{-1}$ : 3100. Anal. Calcd for $\mathrm{C}_{16} \mathrm{H}_{19} \mathrm{~F}_{4} \mathrm{~N}_{3} \mathrm{OS}$ : C, 50.78; H, 5.33; N, 11.10; S, 8.47. Found: C, 50.92; H, 5.07; N, 11.13; S, 8.50.

1,1-Difluoro-1-(methylthio)-2-phenyl-3-(1H-1,2,4-triazol-1-yl)-2propanol (9h): Colorless powder. $\mathrm{mp} 135.8^{\circ} \mathrm{C}$. Yield $38 \%$. ${ }^{1} \mathrm{H}-\mathrm{NMR}$ $\left(\mathrm{CDCl}_{3}\right) \delta: 2.19(3 \mathrm{H}, \mathrm{s}), 4.82(1 \mathrm{H}, \mathrm{d}, J=14.7 \mathrm{~Hz}), 4.87(1 \mathrm{H}, \mathrm{d}, J=14.7 \mathrm{~Hz})$, $6.03(1 \mathrm{H}, \mathrm{s}), 7.30-7.35(3 \mathrm{H}, \mathrm{m}), 7.55-7.58(2 \mathrm{H}, \mathrm{m}), 7.69(1 \mathrm{H}, \mathrm{s}), 7.88$ $(1 \mathrm{H}, \mathrm{s}) .{ }^{19} \mathrm{~F}-\mathrm{NMR}\left(\mathrm{CDCl}_{3}\right) \delta:-8.7(1 \mathrm{~F}, \mathrm{~d}, J=211 \mathrm{~Hz}),-11.4(1 \mathrm{~F}, \mathrm{~d}$, $J=211 \mathrm{~Hz})$. MS $m / z(\%): 285\left(5, \mathrm{M}^{+}\right), 188(100)$. HRMS $m / z: 285.0734$ (Calcd for $\mathrm{C}_{12} \mathrm{H}_{13} \mathrm{~F}_{2} \mathrm{~N}_{3} \mathrm{OS}$ : 285.0748). IR (KBr) cm $\mathrm{cm}^{-1}$ : 3100. Anal. Calcd for $\mathrm{C}_{12} \mathrm{H}_{13} \mathrm{~F}_{2} \mathrm{~N}_{3} \mathrm{OS}$ : C, 50.52; H, 4.58; N, 14.73; S, 11.24. Found: C, 50.70; H, 4.56; N, 14.77; S, 11.34.

1,1-Difluoro-2-(4-fluorophenyl)-1-(methylthio)3-(1H-1,2,4-triazol-1-yl)2-propanol (9i): Colorless powder. mp $123.6{ }^{\circ} \mathrm{C}$. Yield $70 \%$. ${ }^{1} \mathrm{H}-\mathrm{NMR}$ $\left(\mathrm{CDCl}_{3}\right) \delta: 2.23(3 \mathrm{H}, \mathrm{s}), 4.75(1 \mathrm{H}, \mathrm{d}, J=14.2 \mathrm{~Hz}), 4.88(1 \mathrm{H}, \mathrm{d}, J=14.2 \mathrm{~Hz})$, $5.55(1 \mathrm{H}, \mathrm{s}), 7.00-7.05(2 \mathrm{H}, \mathrm{m}), 7.50-7.55(2 \mathrm{H}, \mathrm{m}), 7.81(1 \mathrm{H}, \mathrm{s}), 7.92$ $(1 \mathrm{H}, \mathrm{s}) .{ }^{19} \mathrm{~F}-\mathrm{NMR}\left(\mathrm{CDCl}_{3}\right) \delta:-8.7(1 \mathrm{~F}, \mathrm{dd}, J=7,214 \mathrm{~Hz}),-6.7(1 \mathrm{~F}, \mathrm{dd}$, $J=11,214 \mathrm{~Hz}),-35.8(1 \mathrm{~F}, \mathrm{~s})$. MS $m / z(\%): 303\left(2, \mathrm{M}^{+}\right), 206$ (100). HRMS $m / z$ : 303.0658 (Calcd for $\mathrm{C}_{12} \mathrm{H}_{12} \mathrm{~F}_{3} \mathrm{~N}_{3} \mathrm{OS}: 303.0653$ ). IR (KBr) cm ${ }^{-1}: 3100$. 
Anal. Calcd for $\mathrm{C}_{12} \mathrm{H}_{12} \mathrm{~F}_{3} \mathrm{~N}_{3} \mathrm{OS}$ : C, 47.52; H, 3.99; N, 13.85. Found: C, $47.55 ; \mathrm{H}, 4.13 ; \mathrm{N}, 13.94$.

1,1-Difluoro-1-(methylthio)-3-(1H-1,2,4-triazol-1-yl)-2-[4-(trifluoromethyl)phenyl]-2-propanol $(\mathbf{9 j})$ : Colorless powder. $\mathrm{mp} 188.5^{\circ} \mathrm{C}$. Yield $37 \%$. ${ }^{1} \mathrm{H}-\mathrm{NMR}\left(\mathrm{CDCl}_{3}\right) \delta: 2.24(3 \mathrm{H}, \mathrm{s}), 4.77(1 \mathrm{H}, \mathrm{d}, J=14.6 \mathrm{~Hz}), 4.93(1 \mathrm{H}$, d, $J=14.6 \mathrm{~Hz}), 5.50(1 \mathrm{H}, \mathrm{s}), 7.61(2 \mathrm{H}, \mathrm{d}, J=8.3 \mathrm{~Hz}), 7.67(2 \mathrm{H}, \mathrm{d}, J=8.3 \mathrm{~Hz})$, $7.87(1 \mathrm{H}, \mathrm{s}), 7.95(1 \mathrm{H}, \mathrm{s}) .{ }^{19} \mathrm{~F}-\mathrm{NMR}\left(\mathrm{CDCl}_{3}\right) \delta: 12.9(3 \mathrm{~F}, \mathrm{~s}),-8.5(1 \mathrm{~F}, \mathrm{~d}$, $J=213 \mathrm{~Hz}),-9.3(1 \mathrm{~F}, \mathrm{~d}, J=213 \mathrm{~Hz})$. MS $m / z(\%): 353\left(5, \mathrm{M}^{+}+1\right), 256$ (100). HRMS $m / z 353.0583$ (Calcd for $\mathrm{C}_{13} \mathrm{H}_{12} \mathrm{~F}_{5} \mathrm{~N}_{3} \mathrm{OS}: 353.0621$ ). IR (KBr) $\mathrm{cm}^{-1}: 3100$. Anal. Calcd for $\mathrm{C}_{13} \mathrm{H}_{12} \mathrm{~F}_{5} \mathrm{~N}_{3} \mathrm{OS}: \mathrm{C}, 44.19 ; \mathrm{H}, 3.42 ; \mathrm{N}, 11.89$. Found: C, 44.14; H, 3.57; N, 11.78 .

2-(2,4-Dichlorophenyl)-1,1-difluoro-1-(methylthio)-3-(1H-1,2,4-triazol-1yl)-2-propanol (9k): Colorless powder. mp $143.5^{\circ} \mathrm{C}$. Yield $69 \%$. ${ }^{1} \mathrm{H}-\mathrm{NMR}$ $\left(\mathrm{CDCl}_{3}\right) \delta: 2.28(3 \mathrm{H}, \mathrm{s}), 4.86(1 \mathrm{H}, \mathrm{d}, J=14.7 \mathrm{~Hz}), 5.83(1 \mathrm{H}, \mathrm{d}, J=14.7 \mathrm{~Hz})$, $5.97(1 \mathrm{H}, \mathrm{s}), 7.23(1 \mathrm{H}, \mathrm{dd}, J=2.0,8.8 \mathrm{~Hz}), 7.32(1 \mathrm{H}, \mathrm{d}, J=2.0 \mathrm{~Hz}), 7.83(1 \mathrm{H}$, s), $7.90(1 \mathrm{H}, \mathrm{d}, J=8.8 \mathrm{~Hz}), 8.21(1 \mathrm{H}, \mathrm{s}) .{ }^{19} \mathrm{~F}-\mathrm{NMR}\left(\mathrm{CDCl}_{3}\right) \delta:-6.2(1 \mathrm{~F}, \mathrm{~d}$, $J=210 \mathrm{~Hz}),-8.6(1 \mathrm{~F}, \mathrm{~d}, J=210 \mathrm{~Hz})$. MS $m / z(\%): 353\left(10, \mathrm{M}^{+}\right), 214(100)$. HRMS $m / z$ : 352.9952 (Calcd for $\mathrm{C}_{12} \mathrm{H}_{11} \mathrm{Cl}_{2} \mathrm{~F}_{2} \mathrm{~N}_{3} \mathrm{OS}$ : 352.9968). IR ( $\mathrm{KBr}$ ) $\mathrm{cm}^{-1}$ : 3100. Anal. Calcd for $\mathrm{C}_{12} \mathrm{H}_{11} \mathrm{Cl}_{2} \mathrm{~F}_{2} \mathrm{~N}_{3} \mathrm{OS}$ : C, $40.69 ; \mathrm{H}, 3.13 ; \mathrm{N}, 11.86$; $\mathrm{Cl}, 20.02$. Found: $\mathrm{C}, 40.78 ; \mathrm{H}, 3.18 ; \mathrm{N}, 11.86 ; \mathrm{Cl}, 20.22$.

(+)-9k: Colorless powder, mp $121-123^{\circ} \mathrm{C}$, e.e. $99.3 \%,[\alpha]_{\mathrm{D}}^{25}: 85.0^{\circ}$ $(c=0.03$, methanol), retention time $(\min ): 27.48$.

$(-)-9$ k: Colorless powder, mp $120-123^{\circ} \mathrm{C}$, e.e. $99.1 \%,[\alpha]_{\mathrm{D}}^{25}:-87.5^{\circ}$ $(c=0.02$, methanol), retention time $(\mathrm{min}): 17.26$.

2-[2-(2,4-Difluorophenyl)-1,1-difluoro-2-hydroxy-3-(1H-1,2,4-triazol-1yl)propylthio]ethyl Acetate (9l): Colorless oil. Yield 38\%. ${ }^{1} \mathrm{H}-\mathrm{NMR}\left(\mathrm{CDCl}_{3}\right)$ $\delta: 2.06(3 \mathrm{H}, \mathrm{s}), 3.06(2 \mathrm{H}, \mathrm{t}, J=6.8 \mathrm{~Hz}), 4.24(2 \mathrm{H}, \mathrm{dt}, J=2.0,6.4 \mathrm{~Hz}), 4.81$ $(1 \mathrm{H}, \mathrm{d}, J=14.1 \mathrm{~Hz}), 5.28(1 \mathrm{H}, \mathrm{d}, J=14.1 \mathrm{~Hz}), 5.75(1 \mathrm{H}, \mathrm{s}), 6.71-6.77(1 \mathrm{H}$, $\mathrm{m}), 6.83-6.89(1 \mathrm{H}, \mathrm{m}), 7.71-7.77(1 \mathrm{H}, \mathrm{m}), 7.82(1 \mathrm{H}, \mathrm{s}), 8.09(1 \mathrm{H}, \mathrm{s}) .{ }^{19} \mathrm{~F}-$ NMR $\left(\mathrm{CDCl}_{3}\right) \delta:-8.3(1 \mathrm{~F}, \mathrm{dd}, J=15,210 \mathrm{~Hz}),-8.7(1 \mathrm{~F}, \mathrm{dd}, J=16$, $210 \mathrm{~Hz}),-30.6-30.8(1 \mathrm{~F}, \mathrm{~m}),-32.0-32.1(1 \mathrm{~F}, \mathrm{~m}) . \mathrm{MS} \mathrm{m} / \mathrm{z}(\%): 393$ $\left(0.4, \mathrm{M}^{+}\right), 224(100)$. HRMS $\mathrm{m} / \mathrm{z}: 393.0788$ (Calcd for $\mathrm{C}_{15} \mathrm{H}_{15} \mathrm{~F}_{4} \mathrm{~N}_{3} \mathrm{O}_{2} \mathrm{~S}$ : 393.0770). IR (neat) $\mathrm{cm}^{-1}: 1740,3100$.

2-(2,4-Difluorophenyl)-1,1-difluoro-1-(phenylthio)-3-(1H-1,2,4-triazol-1yl)-2-propanol (9n): Colorless powder. mp $117.3{ }^{\circ} \mathrm{C}$. Yield 51\%. ${ }^{1} \mathrm{H}-\mathrm{NMR}$ $\left(\mathrm{CDCl}_{3}\right) \delta: 4.88(1 \mathrm{H}, \mathrm{d}, J=14.2 \mathrm{~Hz}), 5.30(1 \mathrm{H}, \mathrm{d}, J=14.2 \mathrm{~Hz}), 5.78(1 \mathrm{H}, \mathrm{s})$, $6.71-6.77(1 \mathrm{H}, \mathrm{m}), 6.87-6.89(1 \mathrm{H}, \mathrm{m}), 7.32-7.37(2 \mathrm{H}, \mathrm{m}), 7.40-7.42$ $(1 \mathrm{H}, \mathrm{m}), 7.53-7.56(2 \mathrm{H}, \mathrm{m}), 7.77-7.81(1 \mathrm{H}, \mathrm{m}), 7.83(1 \mathrm{H}, \mathrm{s}), 8.09(1 \mathrm{H}$, s). ${ }^{19} \mathrm{~F}-\mathrm{NMR}\left(\mathrm{CDCl}_{3}\right) \delta:-4.5(1 \mathrm{~F}, \mathrm{dd}, J=26,206 \mathrm{~Hz}),-7.4(1 \mathrm{~F}, \mathrm{dd}, J=16$, $206 \mathrm{~Hz}),-30.5-30.7(1 \mathrm{~F}, \mathrm{~m}),-32.2--32.3(1 \mathrm{~F}, \mathrm{~m})$. FAB-MS $m / z: 384$ $\left(\mathrm{M}+\mathrm{H}^{+}\right)$. IR $(\mathrm{KBr}) \mathrm{cm}^{-1}: 3100$. Anal. Calcd for $\mathrm{C}_{17} \mathrm{H}_{13} \mathrm{~F}_{4} \mathrm{~N}_{3} \mathrm{OS}: \mathrm{C}, 53.26$; H, 3.42; N, 10.96. Found: C, 53.16; H, 3.69; N, 10.86

2-(2,4-Difluorophenyl)-1,1-difluoro-1-[(4-methylphenyl)thio]-3-(1H1,2,4-triazol-1-yl)-2-propanol (9o): Colorless powder. mp 94.1 ${ }^{\circ} \mathrm{C}$. Yield 49\%. ${ }^{1} \mathrm{H}-\mathrm{NMR}\left(\mathrm{CDCl}_{3}\right) \delta: 2.36(3 \mathrm{H}, \mathrm{s}), 4.88(1 \mathrm{H}, \mathrm{d}, J=14.7 \mathrm{~Hz}), 5.29(1 \mathrm{H}$, d, $J=14.7 \mathrm{~Hz}), 5.77(1 \mathrm{H}, \mathrm{s}), 6.70-6.77(1 \mathrm{H}, \mathrm{m}), 6.85-6.90(1 \mathrm{H}, \mathrm{m}), 7.16$ $(2 \mathrm{H}, \mathrm{d}, J=8.3 \mathrm{~Hz}), 7.43(2 \mathrm{H}, \mathrm{d}, J=8.3 \mathrm{~Hz}), 7.76-7.81(1 \mathrm{H}, \mathrm{m}), 7.83(1 \mathrm{H}$, s), $8.08(1 \mathrm{H}, \mathrm{s}) .{ }^{19} \mathrm{~F}-\mathrm{NMR}\left(\mathrm{CDCl}_{3}\right) \delta:-4.8(1 \mathrm{~F}, \mathrm{dd}, J=14,207 \mathrm{~Hz}),-7.8$ (1F, dd, $J=14,207 \mathrm{~Hz}),-30.5--30.6(1 \mathrm{~F}, \mathrm{~m}),-32.2--32.3(1 \mathrm{~F}, \mathrm{~m})$. MS $m / z(\%): 397\left(3, \mathrm{M}^{+}\right), 224$ (100). HRMS $m / z: 397.0865$ (Calcd for $\mathrm{C}_{18} \mathrm{H}_{15} \mathrm{~F}_{4} \mathrm{~N}_{3} \mathrm{OS}:$ 397.0872). IR (KBr) cm ${ }^{-1}$ : 3100. Anal. Calcd for $\mathrm{C}_{18} \mathrm{H}_{15} \mathrm{~F}_{4} \mathrm{~N}_{3} \mathrm{OS}$ : C, 54.40; H, 3.80; N, 10.57. Found: C, 54.54; H, 3.74; N, 10.82 .

1-(Benzylthio)-2-(2,4-difluorophenyl)-1,1-difluoro-3-(1 H-1,2,4-triazol-1yl)-2-propanol (9p): Colorless powder. mp $107.7^{\circ} \mathrm{C}$ Yield $34 \% .{ }^{1} \mathrm{H}-\mathrm{NMR}$ $\left(\mathrm{CDCl}_{3}\right) \delta: 4.03(1 \mathrm{H}, \mathrm{d}, J=12.7 \mathrm{~Hz}), 4.07(1 \mathrm{H}, \mathrm{d}, J=12.7 \mathrm{~Hz}), 4.82(1 \mathrm{H}, \mathrm{d}$, $J=14.7 \mathrm{~Hz}), 5.28(1 \mathrm{H}, \mathrm{d}, J=14.7 \mathrm{~Hz}), 5.73(1 \mathrm{H}, \mathrm{s}), 6.70-6.76(1 \mathrm{H}, \mathrm{m})$, $6.81-6.86(1 \mathrm{H}, \mathrm{m}), 7.24-7.32(5 \mathrm{H}, \mathrm{m}), 7.69-7.76(1 \mathrm{H}, \mathrm{m}), 7.80(1 \mathrm{H}, \mathrm{s})$, $8.08(1 \mathrm{H}, \mathrm{s}) .{ }^{19} \mathrm{~F}-\mathrm{NMR}\left(\mathrm{CDCl}_{3}\right) \delta:-5.9(1 \mathrm{~F}, \mathrm{dd}, J=25,211 \mathrm{~Hz}),-8.7(1 \mathrm{~F}$, $\mathrm{dd}, J=15,211 \mathrm{~Hz}),-30.5--30.7(1 \mathrm{~F}, \mathrm{~m}),-32.2--32.3(1 \mathrm{~F}, \mathrm{~m})$. MS $\mathrm{m} / \mathrm{z}$ (\%): $397\left(1, \mathrm{M}^{+}\right), 224$ (100). HRMS m/z: 397.0849 (Calcd for $\mathrm{C}_{18} \mathrm{H}_{15} \mathrm{~F}_{4} \mathrm{~N}_{3} \mathrm{OS}$ : 397.0872). IR (KBr) $\mathrm{cm}^{-1}:$ 3100. Anal. Calcd for $\mathrm{C}_{18} \mathrm{H}_{15} \mathrm{~F}_{4} \mathrm{~N}_{3} \mathrm{OS}$ : C, 54.40; H, 3.80; N, 10.57. Found: C, 54.46; H, 3.83; N, 10.51 .

2-(2,4-Difluorophenyl)-1,1-difluoro-1-[(4-methoxybenzyl)thio]-3-(1H1,2,4-triazol-1-yl)-2-propanol (9q): Colorless powder. mp $109.5^{\circ} \mathrm{C}$. Yield $63 \%$. ${ }^{1} \mathrm{H}-\mathrm{NMR}\left(\mathrm{CDCl}_{3}\right) \delta: 3.78(3 \mathrm{H}, \mathrm{s}), 4.00(1 \mathrm{H}, \mathrm{d}, J=12.2 \mathrm{~Hz}), 4.04(1 \mathrm{H}$, d, $J=12.2 \mathrm{~Hz}), 4.82(1 \mathrm{H}, \mathrm{d}, J=14.6 \mathrm{~Hz}), 5.28(1 \mathrm{H}, \mathrm{d}, J=14.6 \mathrm{~Hz}), 5.68(1 \mathrm{H}$, s), $6.70-6.74(1 \mathrm{H}, \mathrm{m}), 6.75-6.86(1 \mathrm{H}, \mathrm{m}), 6.82(2 \mathrm{H}, \mathrm{d}, J=8.8 \mathrm{~Hz}), 7.21$ $(2 \mathrm{H}, \mathrm{d}, J=8.8 \mathrm{~Hz}), 7.69-7.75(1 \mathrm{H}, \mathrm{m}), 7.81(1 \mathrm{H}, \mathrm{s}), 8.08(1 \mathrm{H}, \mathrm{s}) .{ }^{19} \mathrm{~F}-\mathrm{NMR}$ $\left(\mathrm{CDCl}_{3}\right) \delta:-6.0(1 \mathrm{~F}, \mathrm{dd}, J=25,211 \mathrm{~Hz}),-8.8(1 \mathrm{~F}, \mathrm{dd}, J=15,211 \mathrm{~Hz})$, $-30.6-30.7$ (1F, m), $-32.2--32.3(1 \mathrm{~F}, \mathrm{~m})$. MS $m / z$ (\%): $427\left(4, \mathrm{M}^{+}\right)$, 121 (100). HRMS $m / z$ : 427.0992 (Calcd for $\mathrm{C}_{19} \mathrm{H}_{17} \mathrm{~F}_{4} \mathrm{~N}_{3} \mathrm{O}_{2} \mathrm{~S}: 427.0978$ ). IR (KBr) $\mathrm{cm}^{-1}$ : 3100. Anal. Calcd for $\mathrm{C}_{19} \mathrm{H}_{17} \mathrm{~F}_{4} \mathrm{~N}_{3} \mathrm{O}_{2} \mathrm{~S}: \mathrm{C}, 53.39$; H, 4.01; N,
9.83. Found: C, $53.45 ; \mathrm{H}, 4.10 ; \mathrm{N}, 9.82$.

Preparation of 2-(2,4-Difluorophenyl)-1,1-difluoro-1-[(2-hydroxyethyl)thiol-3-(1 H-1,2,4-triazol-1-yl)-2-propanol $(9 \mathrm{~m})$ by Hydrolysis of 91 $1 \mathrm{~N} \mathrm{NaOH}(11 \mathrm{ml})$ was added to a solution of $91(10 \mathrm{mmol})$ in dioxane $(50 \mathrm{ml})$ at room temperature with stirring. After the mixture was stirred at the same temperature for $1 \mathrm{~h}$. The mixture was stirred at the same temperature for $15 \mathrm{~h}$. After addition of aqueous ammonium acetate, the mixture was extracted with $\mathrm{Et}_{2} \mathrm{O}$. The organic extract was washed with water and brine, dried over $\mathrm{MgSO}_{4}$, and evaporated under reduced pressure. The residue was chromatographed on a silica gel column using $\mathrm{CHCl}_{3}$ to give $\mathbf{9 m}$. Colorless oil. Yield $90 \%$. ${ }^{1} \mathrm{H}-\mathrm{NMR}\left(\mathrm{CDCl}_{3}\right) \delta: 3.00(2 \mathrm{H}, \mathrm{t}, J=5.8 \mathrm{~Hz}), 3.20(1 \mathrm{H}, \mathrm{br} \mathrm{s})$, $3.78(2 \mathrm{H}, \mathrm{t}, J=5.8 \mathrm{~Hz}), 4.83(1 \mathrm{H}, \mathrm{d}, J=14.6 \mathrm{~Hz}), 5.27(1 \mathrm{H}, \mathrm{d}, J=14.6 \mathrm{~Hz})$, $6.07(1 \mathrm{H}, \mathrm{s}), 6.73-6.79(1 \mathrm{H}, \mathrm{m}), 6.85-6.87(1 \mathrm{H}, \mathrm{m}), 7.68-7.74(1 \mathrm{H}, \mathrm{m})$, $7.76(1 \mathrm{H}, \mathrm{s}), 8.11(1 \mathrm{H}, \mathrm{s}) .{ }^{19} \mathrm{~F}-\mathrm{NMR}\left(\mathrm{CDCl}_{3}\right) \delta$ : $-4.4(1 \mathrm{~F}, \mathrm{dd}, J=28$, $210 \mathrm{~Hz}),-7.9(1 \mathrm{~F}, \mathrm{dd}, J=5,210 \mathrm{~Hz}),-29.8-29.9(1 \mathrm{~F}, \mathrm{~m}),-31.9-$ -32.0 (1F, m). MS $m / z(\%): 352\left(0.5, \mathrm{M}^{+}+1\right), 224(100)$. HRMS $m / z$ : 352.0741 (Calcd for $\mathrm{C}_{13} \mathrm{H}_{14} \mathrm{~F}_{4} \mathrm{~N}_{3} \mathrm{O}_{2} \mathrm{~S}: 352.0743$ ). IR (neat) $\mathrm{cm}^{-1}: 3100$.

General Procedure for Alkylation of $9 \mathrm{~b} \mathrm{NaH}(11 \mathrm{mmol})$ was added to a solution of $9 \mathbf{b}(10 \mathrm{mmol})$ in DMSO $(50 \mathrm{ml})$ at room temperature with stirring. After the mixture was stirred at the same temperature for $1 \mathrm{~h}$, alkyl halide $(55 \mathrm{mmol})$ was added to the solution at room temperature with stirring. The mixture was stirred at the same temperature for $15 \mathrm{~h}$. After addition of aqueous $\mathrm{NH}_{4} \mathrm{Cl}$, the mixture was extracted with $\mathrm{Et}_{2} \mathrm{O}$. The organic extract was washed with water and brine, dried over $\mathrm{MgSO}_{4}$, and evaporated under reduced pressure. The residue was chromatographed on a silica gel column using $\mathrm{CHCl}_{3}$ to give $\mathbf{1 0}$.

1-[2-(2,4-Difluorophenyl)-3-(ethylthio-3,3-difluoro-2-methoxypropyl]$1 H-1,2,4$-triazole (10r): Colorless oil. Yield 68\%. ${ }^{1} \mathrm{H}-\mathrm{NMR}\left(\mathrm{CDCl}_{3}\right) \delta: 1.29$ $(3 \mathrm{H}, \mathrm{t}, J=7.3 \mathrm{~Hz}), 2.75-2.85(2 \mathrm{H}, \mathrm{m}), 3.72(3 \mathrm{H}, \mathrm{d}, J=1.5 \mathrm{~Hz}), 5.05(1 \mathrm{H}, \mathrm{d}$, $J=14.7 \mathrm{~Hz}), 5.15(1 \mathrm{H}, \mathrm{d}, J=14.7 \mathrm{~Hz}), 6.80-6.90(2 \mathrm{H}, \mathrm{m}), 7.60-7.65(1 \mathrm{H}$, $\mathrm{m}), 7.79(1 \mathrm{H}, \mathrm{s}), 7.99(1 \mathrm{H}, \mathrm{s}) .{ }^{19} \mathrm{~F}-\mathrm{NMR}\left(\mathrm{CDCl}_{3}\right) \delta:-0.3(1 \mathrm{~F}, \mathrm{dd}, J=37$, $214 \mathrm{~Hz}),-1.3(1 \mathrm{~F}, \mathrm{dd}, J=22,214 \mathrm{~Hz}),-28.3-28.4(1 \mathrm{~F}, \mathrm{~m}),-32.5-$ -32.6 (1F, m). MS m/z (\%): $349\left(2, \mathrm{M}^{+}\right), 238$ (100). HRMS m/z: 349.0891 (Calcd for $\left.\mathrm{C}_{14} \mathrm{H}_{15} \mathrm{~F}_{4} \mathrm{~N}_{3} \mathrm{OS}: 349.0872\right)$. IR (neat) $\mathrm{cm}^{-1}: 1050$.

1-[2-(Benzyloxy)-2-(2,4-difluorophenyl)-3-(ethylthio)-3,3-difluoropropyl]-1 $\mathrm{H}-1,2,4$-triazole (10s): Colorless oil. Yield 94\%. ${ }^{1} \mathrm{H}-\mathrm{NMR}\left(\mathrm{CDCl}_{3}\right)$ $\delta: 1.31(3 \mathrm{H}, \mathrm{t}, J=7.7 \mathrm{~Hz}), 2.80-2.90(2 \mathrm{H}, \mathrm{m}), 4.93(1 \mathrm{H}, \mathrm{d}, J=10.3 \mathrm{~Hz})$, $5.13(1 \mathrm{H}, \mathrm{d}, J=10.3 \mathrm{~Hz}), 5.18(1 \mathrm{H}, \mathrm{d}, J=15.6 \mathrm{~Hz}), 5.27(1 \mathrm{H}, \mathrm{d}, J=15.6 \mathrm{~Hz})$, $6.75-6.85(2 \mathrm{H}, \mathrm{m}), 7.30-7.45(5 \mathrm{H}, \mathrm{m}), 7.60-7.65(1 \mathrm{H}, \mathrm{m}), 7.79(1 \mathrm{H}, \mathrm{s})$, $7.94(1 \mathrm{H}, \mathrm{s}) .{ }^{19} \mathrm{~F}-\mathrm{NMR}\left(\mathrm{CDCl}_{3}\right) \delta:-0.2(1 \mathrm{~F}, \mathrm{dd}, J=37,214 \mathrm{~Hz}),-0.8(1 \mathrm{~F}$, dd, $J=22,214 \mathrm{~Hz}),-28.3-28.4(1 \mathrm{~F}, \mathrm{~m}),-32.4-32.5(1 \mathrm{~F}, \mathrm{~m})$. MS $\mathrm{m} / \mathrm{z}$ (\%): $426\left(1, \mathrm{M}^{+}+1\right), 238$ (100). HRMS $m / z: 426.1252$ (Calcd for $\mathrm{C}_{20} \mathrm{H}_{20} \mathrm{~F}_{4} \mathrm{~N}_{3}$ OS: 426.1264). IR (KBr) cm $\mathrm{cm}^{-1}: 1070$.

General Procedure for the Synthesis of 1,1-Difluoro-1-(substituted sulfonyl)-3-(1 H-1,2,4-triazol-1-yl)-2-propanol (2) $m$-Chloroperbenzoic acid ( $m$-CPBA) (25 mmol) was added to 2-(substituted thio)-2-propanol (9) $(10 \mathrm{mmol})$ in $\mathrm{CH}_{2} \mathrm{Cl}_{2}(30 \mathrm{ml})$ at room temperature with stirring. The mixture was stirred at room temperature for $5 \mathrm{~h}$. After addition of aqueous $\mathrm{Na}_{2} \mathrm{~S}_{2} \mathrm{O}_{3}$, the mixture was extracted with $\mathrm{CHCl}_{3}$. The organic extract was washed with aqueous $\mathrm{Na}_{2} \mathrm{~S}_{2} \mathrm{O}_{3}$, water and brine, and dried over $\mathrm{MgSO}_{4}$, and evaporated under reduced pressure. The residue was chromatographed on a silica gel column using $\mathrm{CHCl}_{3}-\mathrm{MeOH}[98: 2(\mathrm{v} / \mathrm{v})]$ as a mobile phase to give 2 .

Resolution of $( \pm)-2$ into $(+)-2$ and $(-)-2$ Compound $( \pm)-2(50 \mathrm{mg})$ was subjected to preparative HPLC (CHIRALCEL OD with pre-column CHIRALCEL OD), using $n$-hexane-2-propanol [3:1 (v/v)] as a mobile phase. Their optical yields are measured by HPLC (CHIRALCEL OD with pre-column CHIRALCEL OD) using $n$-hexane-2-propanol $[1: 1(\mathrm{v} / \mathrm{v})]$ as a mobile phase (flow rate: $0.5 \mathrm{ml} / \mathrm{min}$, column temperature: $21^{\circ} \mathrm{C}$ ).

2-(2,4-Difluorophenyl)-1,1-difluoro-1-(methylsulfonyl)-3-(1H-1,2,4-triazol-1-yl)-2-propanol (2a): Colorless powder. mp 104-105 ${ }^{\circ} \mathrm{C}$, Yield $74 \%$. ${ }^{1} \mathrm{H}-\mathrm{NMR}\left(\mathrm{CDCl}_{3}\right) \delta: 3.23(3 \mathrm{H}, \mathrm{s}), 5.20(1 \mathrm{H}, \mathrm{d}, J=14.7 \mathrm{~Hz}), 5.35(1 \mathrm{H}, \mathrm{d}$, $J=14.7 \mathrm{~Hz}), 6.20(1 \mathrm{H}, \mathrm{s}), 6.75-6.90(2 \mathrm{H}, \mathrm{m}), 7.65-7.73(1 \mathrm{H}, \mathrm{m}), 7.76$ $(1 \mathrm{H}, \mathrm{s}), 8.07(1 \mathrm{H}, \mathrm{s}) .{ }^{19} \mathrm{~F}-\mathrm{NMR}\left(\mathrm{CDCl}_{3}\right) \delta:-30.5-30.8(1 \mathrm{~F}, \mathrm{~m}),-31.0-$ $-31.2(1 \mathrm{~F}, \mathrm{~m}),-31.5(1 \mathrm{~F}, \mathrm{dd}, J=13,254 \mathrm{~Hz}),-36.5(1 \mathrm{~F}, \mathrm{dd}, J=29$, $254 \mathrm{~Hz})$. MS $m / z(\%): 354\left(28, \mathrm{M}^{+}+1\right), 141$ (100). HRMS $m / z$ : 354.0546 (Calcd for $\left.\mathrm{C}_{12} \mathrm{H}_{12} \mathrm{~F}_{4} \mathrm{~N}_{3} \mathrm{O}_{3} \mathrm{~S}: 354.0535\right)$. IR (KBr) $\mathrm{cm}^{-1}$ : 3100. Anal. Calcd for $\mathrm{C}_{12} \mathrm{H}_{11} \mathrm{~F}_{4} \mathrm{~N}_{3} \mathrm{O}_{3} \mathrm{~S}$ : C, 40.80; H, 3.14; N, 11.89; S, 9.07. Found: C, 40.93; $\mathrm{H}, 3.14 ; \mathrm{N}, 11.93 ; \mathrm{S}, 9.10$.

$(+)-2 a$ : Colorless powder, $\mathrm{mp} 102-103^{\circ} \mathrm{C}$, e.e. $99.5 \%,[\alpha]_{\mathrm{D}}^{25}: 28.4^{\circ}$ $(c=0.125$, acetone), retention time $(\mathrm{min}): 16.30$.

$(-)-2 a$ : Colorless powder, mp $102-103^{\circ}$ C, e.e. $99.2 \%,[\alpha]_{D}^{25}:-28.0^{\circ}$ $(c=0.25$, acetone), retention time ( $\mathrm{min}): 15.62$.

2-(2,4-Difluorophenyl)-1-(ethylsulfonyl)-1,1-difluoro-3-(1H-1,2,4-triazol1-yl)-2-propanol (2b): Colorless powder. mp $126.3^{\circ} \mathrm{C}$. Yield 78\%. ${ }^{1} \mathrm{H}-\mathrm{NMR}$ 
$\left(\mathrm{CDCl}_{3}\right) \delta: 1.49(3 \mathrm{H}, \mathrm{t}, J=7.7 \mathrm{~Hz}), 3.41(2 \mathrm{H}, \mathrm{q}, J=7.7 \mathrm{~Hz}), 5.19(1 \mathrm{H}, \mathrm{d}$, $J=14.7 \mathrm{~Hz}), 5.35(1 \mathrm{H}, \mathrm{d}, J=14.7 \mathrm{~Hz}), 6.11(1 \mathrm{H}, \mathrm{s}), 6.77-6.93(2 \mathrm{H}, \mathrm{m})$, 7.65-7.74 $(1 \mathrm{H}, \mathrm{m}), 7.76(1 \mathrm{H}, \mathrm{s}), 8.07(1 \mathrm{H}, \mathrm{s}) .{ }^{19} \mathrm{~F}-\mathrm{NMR}\left(\mathrm{CDCl}_{3}\right) \delta$ : $-30.4-30.6(1 \mathrm{~F}, \mathrm{~m}),-31.1-31.3(1 \mathrm{~F}, \mathrm{~m}),-31.1(1 \mathrm{~F}, \mathrm{dd}, J=14$, $254 \mathrm{~Hz}),-35.0$ (1F, dd, $J=29,254 \mathrm{~Hz})$. MS $m / z(\%): 368\left(39, \mathrm{M}^{+}+1\right), 274$ (100). HRMS $m / z$ : 368.0674 (Calcd for $\mathrm{C}_{13} \mathrm{H}_{14} \mathrm{~F}_{4} \mathrm{~N}_{3} \mathrm{O}_{3} \mathrm{~S}: 368.0706$ ). IR $(\mathrm{KBr}) \mathrm{cm}^{-1}$ : 3100. Anal. Calcd for $\mathrm{C}_{13} \mathrm{H}_{13} \mathrm{~F}_{4} \mathrm{~N}_{3} \mathrm{O}_{3} \mathrm{~S}: \mathrm{C}, 42.51 ; \mathrm{H}, 3.57 ; \mathrm{N}$, 11.44. Found: C, $42.69 ; \mathrm{H}, 3.68 ; \mathrm{N}, 11.39$.

$(+)-2 \mathbf{b}$ : Colorless powder, $\mathrm{mp} 131-132^{\circ} \mathrm{C}$, e.e. $100.0 \%,[\alpha]_{\mathrm{D}}^{25}: 23.0^{\circ}$ $(c=0.1$, acetone), retention time $(\mathrm{min}): 16.72$.

(-)-2b: Colorless powder, mp $131-132{ }^{\circ} \mathrm{C}$, e.e. $100.0 \%,[\alpha]_{\mathrm{D}}^{25}:-20.0^{\circ}$ ( $c=0.1$, acetone), retention time ( $\mathrm{min}): 26.00$.

2-(2,4-Difluorophenyl)-1,1-difluoro-1-(propylsulfonyl)-3-(1H-1,2,4-triazol-1-yl)-2-propanol (2c): Colorless powder. mp $113.7^{\circ} \mathrm{C}$. Yield $68 \% .{ }^{1} \mathrm{H}-$ $\operatorname{NMR}\left(\mathrm{CDCl}_{3}\right) \delta: 1.13(3 \mathrm{H}, \mathrm{t}, J=7.3 \mathrm{~Hz}), 1.95-2.05(2 \mathrm{H}, \mathrm{m}), 3.33-3.36$ $(2 \mathrm{H}, \mathrm{m}), 5.19(1 \mathrm{H}, \mathrm{d}, J=14.7 \mathrm{~Hz}), 5.34(1 \mathrm{H}, \mathrm{d}, J=14.7 \mathrm{~Hz}), 6.08(1 \mathrm{H}, \mathrm{s})$, $6.75-6.80(1 \mathrm{H}, \mathrm{m}), 6.83-6.89(1 \mathrm{H}, \mathrm{m}), 7.67-7.78(1 \mathrm{H}, \mathrm{m}), 7.78(1 \mathrm{H}, \mathrm{s})$, $8.07(1 \mathrm{H}, \mathrm{s}) .{ }^{19} \mathrm{~F}-\mathrm{NMR}\left(\mathrm{CDCl}_{3}\right) \delta:-31.1-31.2(1 \mathrm{~F}, \mathrm{~m}),-31.8-31.9$ $(1 \mathrm{~F}, \mathrm{~m}),-32.3(1 \mathrm{~F}, \mathrm{dd}, J=15,254 \mathrm{~Hz}),-36.1(1 \mathrm{~F}, \mathrm{dd}, J=29,254 \mathrm{~Hz})$. MS $m / z(\%): 382\left(3, \mathrm{M}^{+}+1\right), 274$ (100). HRMS $m / z: 382.0974$ (Calcd for $\left.\mathrm{C}_{14} \mathrm{H}_{16} \mathrm{~F}_{4} \mathrm{~N}_{3} \mathrm{O}_{3} \mathrm{~S}: 382.0858\right)$. IR (KBr) cm $\mathrm{cm}^{-1}$ : 3100. Anal. Calcd for $\mathrm{C}_{14} \mathrm{H}_{15} \mathrm{~F}_{4} \mathrm{~N}_{3} \mathrm{O}_{3} \mathrm{~S}$ : C, 48.13; H, 4.33; N, 12.03. Found: C, 48.41; H, 4.34; N, 12.07 .

1-(Cyclopropylsulfonyl)-2-(2,4-difluorophenyl)-1,1-difluoro-3-(1H-1,2,4triazol-1-yl)-2-propanol (2d): Colorless powder. mp $153.3^{\circ} \mathrm{C}$ Yield $76 \%$. ${ }^{1} \mathrm{H}-\mathrm{NMR}\left(\mathrm{CDCl}_{3}\right) \delta: 1.20-1.25(2 \mathrm{H}, \mathrm{m}), 1.40-1.45(2 \mathrm{H}, \mathrm{m}), 2.70-2.75$ $(1 \mathrm{H}, \mathrm{m}), 5.20(1 \mathrm{H}, \mathrm{d}, J=14.7 \mathrm{~Hz}), 5.92(1 \mathrm{H}, \mathrm{d}, J=14.7 \mathrm{~Hz}), 5.92(1 \mathrm{H}, \mathrm{s})$, $6.75-6.80(1 \mathrm{H}, \mathrm{m}), 6.85-6.90(1 \mathrm{H}, \mathrm{m}), 7.70-7.75(1 \mathrm{H}, \mathrm{m}), 7.80(1 \mathrm{H}, \mathrm{s})$, $8.08(1 \mathrm{H}, \mathrm{s}) .{ }^{19} \mathrm{~F}-\mathrm{NMR}\left(\mathrm{CDCl}_{3}\right) \delta:-30.4(1 \mathrm{~F}, \mathrm{dd}, J=18,250 \mathrm{~Hz}),-30.5-$ $-30.6(1 \mathrm{~F}, \mathrm{~m}),-31.4-31.5(1 \mathrm{~F}, \mathrm{~m}),-33.6(1 \mathrm{~F}, \mathrm{dd}, J=29,250 \mathrm{~Hz})$. MS $m / z(\%): 380\left(0.9, \mathrm{M}^{+}+1\right), 274(100)$. HRMS $m / z: 380.0700$ (Calcd for $\mathrm{C}_{14} \mathrm{H}_{14} \mathrm{~F}_{4} \mathrm{~N}_{3} \mathrm{O}_{3} \mathrm{~S}:$ 380.0692). IR (KBr) cm $\mathrm{cm}^{-1}$ : 3100. Anal. Calcd for $\mathrm{C}_{14} \mathrm{H}_{13} \mathrm{~F}_{4} \mathrm{~N}_{3} \mathrm{O}_{3} \mathrm{~S}: \mathrm{C}, 44.33 ; \mathrm{H}, 3.45 ; \mathrm{N}, 11.08$. Found: $\mathrm{C}, 44.20 ; \mathrm{H}, 3.51 ; \mathrm{N}$, 11.06.

$(+)-2 d$ : Colorless powder, $\mathrm{mp} 130-132^{\circ} \mathrm{C}$, e.e. $100.0 \%,[\alpha]_{\mathrm{D}}^{25}: 22.5^{\circ}$ $(c=0.1$, acetone), retention time $(\mathrm{min}): 13.60$.

$(-)-2 d$ : Colorless powder, $\mathrm{mp} 130-132{ }^{\circ} \mathrm{C}$, e.e. $100.0 \%,[\alpha]_{\mathrm{D}}^{25}:-24.5^{\circ}$ $(c=0.1$, acetone), retention time ( $\mathrm{min}): 19.00$.

1-(Butylsulfonyl)-2-(2,4-difluorophenyl)-1,1-difluoro-3-(1H-1,2,4-triazol1-yl)-2-propanol (2e): Colorless powder. mp $84.9^{\circ} \mathrm{C}$. Yield $32 \%$. ${ }^{1} \mathrm{H}-\mathrm{NMR}$ $\left(\mathrm{CDCl}_{3}\right) \delta: 0.98(3 \mathrm{H}, \mathrm{t}, J=7.3 \mathrm{~Hz}), 1.49-1.55(2 \mathrm{H}, \mathrm{m}), 1.89-1.97(2 \mathrm{H}$, m), $3.34-3.38(2 \mathrm{H}, \mathrm{m}), 5.19(1 \mathrm{H}, \mathrm{d}, J=14.2 \mathrm{~Hz}), 5.34(1 \mathrm{H}, \mathrm{d}, J=14.2 \mathrm{~Hz})$, $6.06(1 \mathrm{H}, \mathrm{s}), 6.74-6.80(1 \mathrm{H}, \mathrm{m}), 6.84-6.89(1 \mathrm{H}, \mathrm{m}), 7.65-7.72(1 \mathrm{H}, \mathrm{m})$, $7.78(1 \mathrm{H}, \mathrm{s}), 8.07(1 \mathrm{H}, \mathrm{s}) .{ }^{19} \mathrm{~F}-\mathrm{NMR}\left(\mathrm{CDCl}_{3}\right) \delta:-30.5-30.6(1 \mathrm{~F}, \mathrm{~m})$, $-31.2-31.3(1 \mathrm{~F}, \mathrm{~m}),-31.4(1 \mathrm{~F}, \mathrm{dd}, J=14,254 \mathrm{~Hz}),-35.4(1 \mathrm{~F}, \mathrm{dd}$, $J=29,254 \mathrm{~Hz})$. MS $m / z(\%): 396\left(3, \mathrm{M}^{+}+1\right), 274(100)$. HRMS $m / z$ : 396.1013 (Calcd for $\mathrm{C}_{15} \mathrm{H}_{18} \mathrm{~F}_{4} \mathrm{~N}_{3} \mathrm{O}_{3} \mathrm{~S}: 396.1019$ ). IR (KBr) cm ${ }^{-1}: 3100$. Anal. Calcd for $\mathrm{C}_{15} \mathrm{H}_{17} \mathrm{~F}_{4} \mathrm{~N}_{3} \mathrm{OS}$ : C, 45.57; H, 4.33; N, 10.63. Found: C, 45.60; H, 4.40; N, 10.58 .

1-(tert-Butylsulfonyl)-2-(2,4-difluorophenyl)-1,1-difluoro-3-(1H-1,2,4-triazol-1-yl)-2-propanol (2f): Colorless powder. mp $155.1^{\circ} \mathrm{C}$. Yield $71 \% .{ }^{1} \mathrm{H}-$ NMR $\left(\mathrm{CDCl}_{3}\right) \delta: 2.18(9 \mathrm{H}, \mathrm{s}), 5.17(1 \mathrm{H}, \mathrm{d}, J=14.2 \mathrm{~Hz}), 5.31(1 \mathrm{H}, \mathrm{d}$, $J=14.2 \mathrm{~Hz}), 5.66(1 \mathrm{H}, \mathrm{s}), 6.76-6.84(1 \mathrm{H}, \mathrm{m}), 6.86-6.89(1 \mathrm{H}, \mathrm{m}), 7.66-$ $7.72(1 \mathrm{H}, \mathrm{m}), 7.79(1 \mathrm{H}, \mathrm{s}), 8.13(1 \mathrm{H}, \mathrm{s}) .{ }^{19} \mathrm{~F}-\mathrm{NMR}\left(\mathrm{CDCl}_{3}\right) \delta:-25.0(1 \mathrm{~F}$, dd, $J=24,238 \mathrm{~Hz}),-26.1(1 \mathrm{~F}, \mathrm{dd}, J=24,238 \mathrm{~Hz}),-29.3-29.4(1 \mathrm{~F}, \mathrm{~m})$, -31.6--31.7 (1F, m). MS $m / z$ (\%): $396\left(3, \mathrm{M}^{+}+1\right), 274$ (100). HRMS $m / z: 396.0995$ (Calcd for $\mathrm{C}_{15} \mathrm{H}_{18} \mathrm{~F}_{4} \mathrm{~N}_{3} \mathrm{O}_{3} \mathrm{~S}: 396.1019$ ). IR (KBr) cm ${ }^{-1}: 3100$. Anal. Calcd for $\mathrm{C}_{15} \mathrm{H}_{17} \mathrm{~F}_{4} \mathrm{~N}_{3} \mathrm{OS}$ : C, 45.57; H, 4.33; N, 10.63. Found: C, $45.79 ; \mathrm{H}, 4.45 ; \mathrm{N}, 10.56$.

2-(2,4-Difluorophenyl)-1,1-difluoro-1-(pentylsulfonyl)-3-(1H-1,2,4-triazol-1-yl)-2-propanol (2g): Colorless powder. mp $78.2{ }^{\circ} \mathrm{C}$. Yield $46 \% .{ }^{1} \mathrm{H}-$ NMR $\left(\mathrm{CDCl}_{3}\right) \delta: 0.98(3 \mathrm{H}, \mathrm{t}, J=7.3 \mathrm{~Hz}), 1.17-1.90(6 \mathrm{H}, \mathrm{m}), 3.36-3.40$ $(2 \mathrm{H}, \mathrm{m}), 5.19(1 \mathrm{H}, \mathrm{d}, J=14.2 \mathrm{~Hz}), 5.34(1 \mathrm{H}, \mathrm{d}, J=14.2 \mathrm{~Hz}), 6.06(1 \mathrm{H}, \mathrm{s})$, $6.74-6.80(1 \mathrm{H}, \mathrm{m}), 6.84-6.89(1 \mathrm{H}, \mathrm{m}), 7.65-7.72(1 \mathrm{H}, \mathrm{m}), 7.78(1 \mathrm{H}, \mathrm{s})$, $8.07(1 \mathrm{H}, \mathrm{s}) .{ }^{19} \mathrm{~F}-\mathrm{NMR}\left(\mathrm{CDCl}_{3}\right) \delta:-30.5-30.6(1 \mathrm{~F}, \mathrm{~m}),-31.2--31.3$ $(1 \mathrm{~F}, \mathrm{~m}),-31.4(1 \mathrm{~F}, \mathrm{dd}, J=14,254 \mathrm{~Hz}),-35.4(1 \mathrm{~F}, \mathrm{dd}, J=29,254 \mathrm{~Hz})$. MS $m / z(\%): 410\left(2, \mathrm{M}^{+}+1\right), 274(100)$. HRMS $m / z: 410.1139$ (Calcd for $\mathrm{C}_{16} \mathrm{H}_{20} \mathrm{~F}_{4} \mathrm{~N}_{3} \mathrm{O}_{3} \mathrm{~S}:$ 410.1161). IR (KBr) cm $\mathrm{cm}^{-1}$ : 3100. Anal. Calcd for $\mathrm{C}_{16} \mathrm{H}_{19} \mathrm{~F}_{4} \mathrm{~N}_{3} \mathrm{O}_{3} \mathrm{~S}: \mathrm{C}, 46.94 ; \mathrm{H}, 4.68 ; \mathrm{N}, 10.26$. Found: $\mathrm{C}, 46.710 ; \mathrm{H}, 4.82 ; \mathrm{N}$, 10.13 .

1,1-Difluoro-1-(methylsulfonyl)-2-phenyl-3-(1H-1,2,4-triazol-1-yl)-2propanol (2h): Colorless powder. mp $124.0^{\circ} \mathrm{C}$. Yield $46 \%$. ${ }^{1} \mathrm{H}-\mathrm{NMR}$ $\left(\mathrm{CDCl}_{3}\right) \delta: 3.14(3 \mathrm{H}, \mathrm{s}), 4.92(1 \mathrm{H}, \mathrm{d}, J=14.2 \mathrm{~Hz}), 5.28(1 \mathrm{H}, \mathrm{d}, J=14.2 \mathrm{~Hz})$, $6.2(1 \mathrm{H}, \mathrm{s}), 7.30-7.40(3 \mathrm{H}, \mathrm{m}), 7.50-7.56(2 \mathrm{H}, \mathrm{m}), 7.72(1 \mathrm{H}, \mathrm{s}), 7.91(1 \mathrm{H}$, s). ${ }^{19} \mathrm{~F}-\mathrm{NMR}\left(\mathrm{CDCl}_{3}\right) \delta:-30.7(1 \mathrm{~F}, \mathrm{~d}, J=256 \mathrm{~Hz}),-35.5(1 \mathrm{~F}, \mathrm{~d}, J=256 \mathrm{~Hz})$. MS $m / z(\%): 318\left(2, \mathrm{M}^{+}+1\right), 238$ (100). HRMS $m / z$ : 318.0711 (Calcd for $\mathrm{C}_{12} \mathrm{H}_{14} \mathrm{~F}_{2} \mathrm{~N}_{3} \mathrm{O}_{3} \mathrm{~S}$ : 318.0725). IR (KBr) $\mathrm{cm}^{-1}$ : 3100. Anal. Calcd for $\mathrm{C}_{12} \mathrm{H}_{13} \mathrm{~F}_{2} \mathrm{~N}_{3} \mathrm{O}_{3} \mathrm{~S}$ : C, 45.42; H, 4.13; N, 13.24. Found: C, 45.34; H, 4.23; N, 13.21 .

1,1-Difluoro-2-(4-fluorophenyl)-1-(methylsulfonyl)-3-(1H-1,2,4-triazol-1yl)-2-propanol (2i): Colorless powder. mp $168.9^{\circ} \mathrm{C}$. Yield $33 \%$. ${ }^{1} \mathrm{H}-\mathrm{NMR}$ $\left(\mathrm{CDCl}_{3}\right) \delta: 3.16(3 \mathrm{H}, \mathrm{s}), 4.86(1 \mathrm{H}, \mathrm{d}, J=14.2 \mathrm{~Hz}), 5.28(1 \mathrm{H}, \mathrm{d}, J=14.2 \mathrm{~Hz})$, $6.00(1 \mathrm{H}, \mathrm{s}), 7.00-7.05(2 \mathrm{H}, \mathrm{m}), 7.45-7.50(2 \mathrm{H}, \mathrm{m}), 7.79(1 \mathrm{H}, \mathrm{s}), 7.88$ $(1 \mathrm{H}, \mathrm{s}) .{ }^{19} \mathrm{~F}-\mathrm{NMR}\left(\mathrm{CDCl}_{3}\right) \delta:-8.7(1 \mathrm{~F}, \mathrm{dd}, J=7,256 \mathrm{~Hz}),-6.7(1 \mathrm{~F}, \mathrm{dd}$, $J=11,256 \mathrm{~Hz}),-35.8(1 \mathrm{~F}, \mathrm{~s})$. MS $m / z(\%): 336\left(1, \mathrm{M}^{+}+1\right), 256(94), 123$ (100). HRMS $m / z$ : 336.0635 (Calcd for $\mathrm{C}_{12} \mathrm{H}_{13} \mathrm{~F}_{3} \mathrm{~N}_{3} \mathrm{O}_{3} \mathrm{~S}: 336.0630$ ). IR (KBr) $\mathrm{cm}^{-1}$ : 3050. Anal. Calcd for $\mathrm{C}_{12} \mathrm{H}_{12} \mathrm{~F}_{3} \mathrm{~N}_{3} \mathrm{O}_{3} \mathrm{~S}: \mathrm{C}, 42.98 ; \mathrm{H}, 3.61 ; \mathrm{N}$, 12.53; S, 9.56. Found: C, 43.09; H, 3.63; N, 12.57; S, 9.56 .

$(+)-2 \mathrm{i}$ : Colorless powder, mp $103-106^{\circ} \mathrm{C}$, e.e. $99.0 \%,[\alpha]_{\mathrm{D}}^{25}: 27.0^{\circ}$ $(c=0.1$, methanol), retention time $(\mathrm{min}): 13.70$.

$(-)-2 \mathbf{i}$ : Colorless powder, mp $102-105^{\circ} \mathrm{C}$, e.e. $99.8 \%,[\alpha]_{\mathrm{D}}^{25}:-31.0^{\circ}$ $(c=0.1$, methanol), retention time $(\mathrm{min}): 20.23$.

1,1-Difluoro-1-(methylsulfonyl)-3-(1H-1,2,4-triazol-1-yl)-2-[4-(trifluoromethyl)phenyl]-2-propanol (2j): Colorless powder. $\mathrm{mp} 188.7^{\circ} \mathrm{C}$. Yield $53 \%$. ${ }^{1} \mathrm{H}-\mathrm{NMR}\left(\mathrm{CDCl}_{3}\right) \delta: 3.19(3 \mathrm{H}, \mathrm{s}), 3.56(1 \mathrm{H}, \mathrm{s}), 5.03(1 \mathrm{H}, \mathrm{d}$, $J=14.7 \mathrm{~Hz}), 5.25(1 \mathrm{H}, \mathrm{d}, J=14.7 \mathrm{~Hz}), 7.60(2 \mathrm{H}, \mathrm{d}, J=8.8 \mathrm{~Hz}), 7.66(2 \mathrm{H}, \mathrm{d}$, $J=8.8 \mathrm{~Hz}), 7.73(1 \mathrm{H}, \mathrm{s}), 8.06(1 \mathrm{H}, \mathrm{s}) .{ }^{19} \mathrm{~F}-\mathrm{NMR}\left(\mathrm{CDCl}_{3}\right) \delta: 12.7(3 \mathrm{~F}, \mathrm{~s})$, $-31.4(1 \mathrm{~F}, \mathrm{~d}, J=254 \mathrm{~Hz}),-34.4(1 \mathrm{~F}, \mathrm{~d}, J=254 \mathrm{~Hz})$. MS $\mathrm{m} / z$ (\%): $386(0.9$, $\left.\mathrm{M}^{+}+1\right), 366\left(7, \mathrm{M}^{+}-\mathrm{F}\right), 306$ (100). HRMS m/z: 366.0504 (Calcd for $\mathrm{C}_{13} \mathrm{H}_{12} \mathrm{~F}_{4} \mathrm{~N}_{3} \mathrm{O}_{3} \mathrm{~S}:$ 366.0535). IR (KBr) $\mathrm{cm}^{-1}: 3100$. Anal. Calcd for $\mathrm{C}_{13} \mathrm{H}_{12} \mathrm{~F}_{5} \mathrm{~N}_{3} \mathrm{O}_{3} \mathrm{~S}$ : C, 40.52; H, 3.14; N, 10.91. Found: C, 40.30; H, 3.09; N, 10.73 .

$(+)-2 \mathbf{j}$ : Colorless powder, mp $147-150{ }^{\circ} \mathrm{C}$, e.e. $99.5 \%,[\alpha]_{\mathrm{D}}^{25}: 20.5^{\circ}$ $(c=0.1$, methanol), retention time $(\min ): 13.20$.

$(-)-2 \mathbf{j}$ : Colorless powder, mp $153-154^{\circ} \mathrm{C}$, e.e. $99.5 \%,[\alpha]_{\mathrm{D}}^{25}:-20.0^{\circ}$ $(c=0.1$, methanol), retention time $(\mathrm{min}): 21.72$.

2-(2,4-Dichlorophenyl)-1,1-difluoro-1-(methylsulfonyl)-3-(1 H-1,2,4-triazol-1-yl)-2-propanol (2k): Colorless powder. mp $146.7^{\circ} \mathrm{C}$. Yield $91 \% .{ }^{1} \mathrm{H}-$ $\operatorname{NMR}\left(\mathrm{CDCl}_{3}\right) \delta: 3.23(3 \mathrm{H}, \mathrm{s}), 5.21(1 \mathrm{H}, \mathrm{d}, J=14.6 \mathrm{~Hz}), 5.99(1 \mathrm{H}, \mathrm{d}$, $J=14.6 \mathrm{~Hz}), 6.40(1 \mathrm{H}, \mathrm{s}), 7.23(1 \mathrm{H}, \mathrm{dd}, J=2.0,8.8 \mathrm{~Hz}), 7.35(1 \mathrm{H}, \mathrm{d}$, $J=2.0 \mathrm{~Hz}), 7.81(1 \mathrm{H}, \mathrm{s}), 7.83(1 \mathrm{H}, \mathrm{d}, J=8.8 \mathrm{~Hz}), 8.16(1 \mathrm{H}, \mathrm{s}) .{ }^{19} \mathrm{~F}-\mathrm{NMR}$ $\left(\mathrm{CDCl}_{3}\right) \delta:-29.0(1 \mathrm{~F}, \mathrm{~d}, J=248 \mathrm{~Hz}),-32.3(1 \mathrm{~F}, \mathrm{~d}, J=248 \mathrm{~Hz})$. MS $m / z(\%)$ : $386\left(1, \quad \mathrm{M}^{+}+1\right), 306 \quad(100)$. HRMS $m / z: 385.9921$ (Calcd for $\left.\mathrm{C}_{12} \mathrm{H}_{12} \mathrm{Cl}_{2} \mathrm{~F}_{2} \mathrm{~N}_{3} \mathrm{O}_{3} \mathrm{~S}: 385.9944\right)$. IR ( $\left.\mathrm{KBr}\right) \mathrm{cm}^{-1}: 3100$. Anal. Calcd for $\mathrm{C}_{12} \mathrm{H}_{11} \mathrm{Cl}_{2} \mathrm{~F}_{2} \mathrm{~N}_{3} \mathrm{O}_{3} \mathrm{~S}$ : C, 37.32; H, 2.87; N, 10.88; Cl, 18.36. Found: C, 37.43; $\mathrm{H}, 2.91 ; \mathrm{N}, 10.91 ; \mathrm{Cl}, 18.00$.

$(+)-2 \mathrm{k}$ : Colorless powder, $\mathrm{mp} 165-167^{\circ} \mathrm{C}$, e.e. $100.0 \%,[\alpha]_{\mathrm{D}}^{25}: 30.2^{\circ}$ $(c=0.04$, methanol), retention time $(\mathrm{min}): 25.91$.

$(-)-2 k$ : Colorless powder, mp $165-167{ }^{\circ} \mathrm{C}$, e.e. $96.9 \%,[\alpha]_{\mathrm{D}}^{25}:-29.5^{\circ}$ $(c=0.1$, methanol), retention time $(\mathrm{min}): 22.51$.

2-(2,4-Difluorophenyl)-1,1-difluoro-1-[(2-hydroxyethyl)sulfonyl]-3-(1H1,2,4-triazol-1-yl)-2-propanol (2m): Colorless powder. $\mathrm{mp} 77-80^{\circ} \mathrm{C}$. Yield 14\%. ${ }^{1} \mathrm{H}-\mathrm{NMR}\left(\mathrm{CDCl}_{3}\right) \delta: 3.70(2 \mathrm{H}, \mathrm{t}, J=5.4 \mathrm{~Hz}), 4.20-4.25(2 \mathrm{H}, \mathrm{m}), 5.19$ $(1 \mathrm{H}, \mathrm{d}, J=14.2 \mathrm{~Hz}), 5.35(1 \mathrm{H}, \mathrm{d}, J=14.2 \mathrm{~Hz}), 6.15(1 \mathrm{H}, \mathrm{s}), 6.75-6.79(1 \mathrm{H}$, $\mathrm{m}), 6.80-6.89(1 \mathrm{H}, \mathrm{m}), 7.64-7.70(1 \mathrm{H}, \mathrm{m}), 7.81(1 \mathrm{H}, \mathrm{s}), 8.06(1 \mathrm{H}, \mathrm{s}) .{ }^{19} \mathrm{~F}-$ NMR $\left(\mathrm{CDCl}_{3}\right) \delta:-30.8-30.9(2 \mathrm{~F}, \mathrm{~m}),-31.2(1 \mathrm{~F}, \mathrm{dd}, J=13,252 \mathrm{~Hz})$, -36.8 ( $1 \mathrm{~F}, \mathrm{dd}, J=33,252 \mathrm{~Hz}$ ). MS $m / z(\%): 384\left(1, \mathrm{M}^{+}+1\right), 274(100)$. HRMS $m / z$ : 384.0627 (Calcd for $\mathrm{C}_{13} \mathrm{H}_{14} \mathrm{~F}_{4} \mathrm{~N}_{3} \mathrm{O}_{4} \mathrm{~S}: 384.0641$ ). IR (neat) $\mathrm{cm}^{-1}$ : 3100. Anal. Calcd for $\mathrm{C}_{13} \mathrm{H}_{13} \mathrm{~F}_{4} \mathrm{~N}_{3} \mathrm{O}_{4} \mathrm{~S}: \mathrm{C}, 40.73 ; \mathrm{H}, 3.42 ; \mathrm{N}, 10.96$. Found: C, 40.51; H, 3.45; N, 10.96 .

2-(2,4-Difluorophenyl)-1,1-difluoro-1-(phenylsulfonyl)-3-(1H-1,2,4-triazol-1-yl)-2-propanol (2n) Colorless powder. mp $123.4^{\circ} \mathrm{C}$. Yield $49 \% .{ }^{1} \mathrm{H}-$ $\operatorname{NMR}\left(\mathrm{CDCl}_{3}\right) \delta: 5.27(1 \mathrm{H}, \mathrm{d}, J=14.7 \mathrm{~Hz}), 5.36(1 \mathrm{H}, \mathrm{d}, J=14.7 \mathrm{~Hz}), 6.71-$ $6.77(1 \mathrm{H}, \mathrm{m}), 6.83-6.88(1 \mathrm{H}, \mathrm{m}), 7.59-7.63(2 \mathrm{H}, \mathrm{m}), 7.70-7.82(2 \mathrm{H}, \mathrm{m})$, $7.81(1 \mathrm{H}, \mathrm{s}), 7.97-8.00(2 \mathrm{H}, \mathrm{m}), 8.10(1 \mathrm{H}, \mathrm{s}) .{ }^{19} \mathrm{~F}-\mathrm{NMR}\left(\mathrm{CDCl}_{3}\right) \delta:-29.3$ $(1 \mathrm{~F}, \mathrm{dd}, J=11,253 \mathrm{~Hz}),-30.0--30.1(1 \mathrm{~F}, \mathrm{~m}),-30.2(1 \mathrm{~F}, \mathrm{dd}, J=25$, $253 \mathrm{~Hz}),-31.1-31.2(1 \mathrm{~F}, \mathrm{~m})$. MS $m / z(\%): 416\left(1, \mathrm{M}^{+}+1\right), 274(100)$. HRMS $m / z: 416.0718$ (Calcd for $\mathrm{C}_{17} \mathrm{H}_{14} \mathrm{~F}_{4} \mathrm{~N}_{3} \mathrm{O}_{3} \mathrm{~S}: 416.0691$ ). IR (neat) $\mathrm{cm}^{-1}$ : 3100. Anal. Calcd for $\mathrm{C}_{17} \mathrm{H}_{13} \mathrm{~F}_{4} \mathrm{~N}_{3} \mathrm{O}_{3} \mathrm{~S}: \mathrm{C}, 49.16 ; \mathrm{H}, 3.15 ; \mathrm{N}, 10.12$. Found: C, 49.23; H, 3.15; N, 10.11 .

2-(2,4-Difluorophenyl)-1,1-difluoro-1-[(4-methylphenyl)sulfonyl]-3-(1H1,2,4-triazol-1-yl)-2-propanol (2o): Colorless powder. $\mathrm{mp} 108.9^{\circ} \mathrm{C}$. Yield $63 \%$. ${ }^{1} \mathrm{H}-\mathrm{NMR}\left(\mathrm{CDCl}_{3}\right) \delta: 2.48(3 \mathrm{H}, \mathrm{s}), 5.26(1 \mathrm{H}, \mathrm{d}, J=14.7 \mathrm{~Hz}), 5.36(1 \mathrm{H}$, d, $J=14.7 \mathrm{~Hz}), 5.80(1 \mathrm{H}, \mathrm{s}), 6.71-6.77(1 \mathrm{H}, \mathrm{m}), 6.82-6.88(1 \mathrm{H}, \mathrm{m}), 7.40$ $(2 \mathrm{H}, \mathrm{d}, J=8.3 \mathrm{~Hz}), 7.68-7.74(1 \mathrm{H}, \mathrm{m}), 7.81(1 \mathrm{H}, \mathrm{s}), 7.85(2 \mathrm{H}, \mathrm{d}$, $J=8.3 \mathrm{~Hz}), 8.11(1 \mathrm{H}, \mathrm{s}) .{ }^{19} \mathrm{~F}-\mathrm{NMR}\left(\mathrm{CDCl}_{3}\right) \delta:-30.0 \quad(1 \mathrm{~F}, \mathrm{dd}, J=21$, $249 \mathrm{~Hz}),-30.1--30.3(1 \mathrm{~F}, \mathrm{~m}),-30.8(1 \mathrm{~F}, \mathrm{dd}, J=19,249 \mathrm{~Hz}),-31.4-$ $-31.6(1 \mathrm{~F}, \mathrm{~m})$. MS $m / z(\%): 430\left(0.5, \mathrm{M}^{+}+1\right), 274(100)$. HRMS $m / z$ : 
430.0833 (Calcd for $\mathrm{C}_{18} \mathrm{H}_{16} \mathrm{~F}_{4} \mathrm{~N}_{3} \mathrm{O}_{3} \mathrm{~S}$ : 430.0863). IR (KBr) cm $\mathrm{cm}^{-1}: 3100$. Anal. Calcd for $\mathrm{C}_{18} \mathrm{H}_{15} \mathrm{~F}_{4} \mathrm{~N}_{3} \mathrm{O}_{3} \mathrm{~S}$ : C, 50.35; H, 3.52; N, 9.79. Found: C, $50.43 ; \mathrm{H}, 3.60 ; \mathrm{N}, 9.88$.

1-(Benzylsulfonyl)-2-(2,4-difluorophenyl)-1,1-difluoro-3-(1H-1,2,4-triazol-1-yl)-2-propanol (2p): Colorless powder. mp $138.6^{\circ} \mathrm{C}$ Yield $64 \% .{ }^{1} \mathrm{H}-$ $\operatorname{NMR}\left(\mathrm{CDCl}_{3}\right) \delta: 4.59(1 \mathrm{H}, \mathrm{d}, J=13.2 \mathrm{~Hz}), 4.64(1 \mathrm{H}, \mathrm{d}, J=13.2 \mathrm{~Hz}), 5.23$ $(1 \mathrm{H}, \mathrm{d}, J=14.7 \mathrm{~Hz}), 5.35(1 \mathrm{H}, \mathrm{d}, J=14.7 \mathrm{~Hz}), 6.19(1 \mathrm{H}, \mathrm{s}), 6.74-6.79(1 \mathrm{H}$, m), $6.84-6.89(1 \mathrm{H}, \mathrm{m}), 7.42(5 \mathrm{H}, \mathrm{s}), 7.68-7.75(1 \mathrm{H}, \mathrm{m}), 7.79(1 \mathrm{H}, \mathrm{s}), 8.07$ $(1 \mathrm{H}, \mathrm{s}) .{ }^{19} \mathrm{~F}-\mathrm{NMR}\left(\mathrm{CDCl}_{3}\right) \delta:-38.5(1 \mathrm{~F}, \mathrm{dd}, J=14,253 \mathrm{~Hz}),-41.9(1 \mathrm{~F}, \mathrm{dd}$, $J=17,253 \mathrm{~Hz}),-38.4-38.7(1 \mathrm{~F}, \mathrm{~m}),-39.0-39.1(1 \mathrm{~F}, \mathrm{~m}) . \mathrm{MS} \mathrm{m} / \mathrm{z}$ (\%): $430\left(1, \mathrm{M}^{+}+1\right), 274$ (52), 91 (100). HRMS $m / z$ : 430.0834 (Calcd for $\mathrm{C}_{18} \mathrm{H}_{16} \mathrm{~F}_{4} \mathrm{~N}_{3} \mathrm{O}_{3} \mathrm{~S}:$ 430.0863). IR (KBr) $\mathrm{cm}^{-1}$ : 3100. Anal. Calcd for $\mathrm{C}_{18} \mathrm{H}_{15} \mathrm{~F}_{4} \mathrm{~N}_{3} \mathrm{O}_{3} \mathrm{~S}$ : C, 50.35; H, 3.52; N, 9.79. Found: C, 50.51; H, 3.69; N, 9.81 .

2-(2,4-Difluorophenyl)-1,1-difluoro-1-[(4-methoxyphenyl)methylsulfonyl]-3-(1H-1,2,4-triazol-1-yl)-2-propanol (2q): Colorless powder. $\mathrm{mp}$ $79.0^{\circ} \mathrm{C}$. Yield $37 \%$. ${ }^{1} \mathrm{H}-\mathrm{NMR}\left(\mathrm{CDCl}_{3}\right) \delta: 3.81(3 \mathrm{H}, \mathrm{s}), 4.53(1 \mathrm{H}, \mathrm{d}$, $J=13.4 \mathrm{~Hz}), 4.59(1 \mathrm{H}, \mathrm{d}, J=13.4 \mathrm{~Hz}), 5.22(1 \mathrm{H}, \mathrm{d}, J=14.7 \mathrm{~Hz}), 5.35(1 \mathrm{H}, \mathrm{d}$, $J=14.7 \mathrm{~Hz}), 6.17(1 \mathrm{H}, \mathrm{s}), 6.74-6.80(1 \mathrm{H}, \mathrm{m}), 6.84-6.90(1 \mathrm{H}, \mathrm{m}), 6.93$ $(2 \mathrm{H}, \mathrm{d}, J=8.3 \mathrm{~Hz}), 7.34(2 \mathrm{H}, \mathrm{d}, J=8.3 \mathrm{~Hz}), 7.68-7.74(1 \mathrm{H}, \mathrm{m}), 7.80(1 \mathrm{H}$, s), $8.09(1 \mathrm{H}, \mathrm{s}) .{ }^{19} \mathrm{~F}-\mathrm{NMR}\left(\mathrm{CDCl}_{3}\right) \delta:-30.3--30.5(1 \mathrm{~F}, \mathrm{~m}),-30.6(1 \mathrm{~F}$, dd, $J=14,254 \mathrm{~Hz}),-30.9-31.2(1 \mathrm{~F}, \mathrm{~m}),-34.0(1 \mathrm{~F}, \mathrm{dd}, J=27,254 \mathrm{~Hz})$. MS $m / z(\%): 459\left(0.2, \mathrm{M}^{+}\right), 121(100)$. HRMS $m / z$ : 459.0903 (Calcd for $\mathrm{C}_{19} \mathrm{H}_{17} \mathrm{~F}_{4} \mathrm{~N}_{3} \mathrm{O}_{4} \mathrm{~S}:$ 459.0876). IR (KBr) cm $\mathrm{cm}^{-1}$ : 3100. Anal. Calcd for $\mathrm{C}_{19} \mathrm{H}_{17} \mathrm{~F}_{4} \mathrm{~N}_{3} \mathrm{O}_{4} \mathrm{~S}$ : C, 49.67; H, 3.73; N, 9.15; S, 6.98. Found: C, 49.59; H, $3.82 ; \mathrm{N}, 9.15$.

1-[2-(2,4-Difluorophenyl)-3-(ethylsulfonyl)-3,3-difluoro-2-methoxypropyl]-1H-1,2,4-triazole (11r): Colorless oil. Yield $78 \%$. ${ }^{1} \mathrm{H}-\mathrm{NMR}\left(\mathrm{CDCl}_{3}\right)$ $\delta: 1.43(3 \mathrm{H}, \mathrm{t}, J=7.3 \mathrm{~Hz}), 3.17-3.23(2 \mathrm{H}, \mathrm{m}), 3.74(3 \mathrm{H}, \mathrm{d}, J=1.2 \mathrm{~Hz}), 5.16$ $(1 \mathrm{H}, \mathrm{d}, J=15.6 \mathrm{~Hz}), 5.32(1 \mathrm{H}, \mathrm{d}, J=15.6 \mathrm{~Hz}), 6.78-6.90(2 \mathrm{H}, \mathrm{m}), 7.53-$ $7.60(1 \mathrm{H}, \mathrm{m}), 7.78(1 \mathrm{H}, \mathrm{s}), 8.06(1 \mathrm{H}, \mathrm{s}) .{ }^{19} \mathrm{~F}-\mathrm{NMR}\left(\mathrm{CDCl}_{3}\right) \delta:-25.3(1 \mathrm{~F}$, dd, $J=30,248 \mathrm{~Hz}),-26.8(1 \mathrm{~F}, \mathrm{dd}, J=16,248 \mathrm{~Hz}),-28.0-28.1(1 \mathrm{~F}, \mathrm{~m})$, $-31.2--31.3(1 \mathrm{~F}, \mathrm{~m})$. MS $m / z$ (\%): $382\left(0.5, \mathrm{M}^{+}+1\right), 288$ (100). HRMS $\mathrm{m} / z$ : 382.0834 (Calcd for $\left.\mathrm{C}_{14} \mathrm{H}_{16} \mathrm{~F}_{4} \mathrm{~N}_{3} \mathrm{O}_{3} \mathrm{~S}: 382.0848\right)$. IR $(\mathrm{KBr}) \mathrm{cm}^{-1}: 1050$.

1-[2-(Benzyloxy)-2-(2,4-difluorophenyl)-3-ethylsulfonyl-3,3-difluoropropyl]-1 $H-1,2,4$-triazole (11s): Colorless powder. mp $117.3^{\circ} \mathrm{C}$. Yield $33 \%$. ${ }^{1} \mathrm{H}-\mathrm{NMR}\left(\mathrm{CDCl}_{3}\right) \delta: 1.41(3 \mathrm{H}, \mathrm{t}, J=7.7 \mathrm{~Hz}), 3.15-3.21(2 \mathrm{H}, \mathrm{m}), 5.05(1 \mathrm{H}$, d, $J=9.8 \mathrm{~Hz}), 5.10(1 \mathrm{H}, \mathrm{d}, J=9.8 \mathrm{~Hz}), 5.30(1 \mathrm{H}, \mathrm{d}, J=15.6 \mathrm{~Hz}), 5.47(1 \mathrm{H}, \mathrm{d}$, $J=15.6 \mathrm{~Hz}), 6.80-6.90(2 \mathrm{H}, \mathrm{m}), 7.34-7.41(3 \mathrm{H}, \mathrm{m}), 7.45-7.47(2 \mathrm{H}, \mathrm{m})$, $7.58-7.62(1 \mathrm{H}, \mathrm{m}), 7.80(1 \mathrm{H}, \mathrm{s}), 8.04(1 \mathrm{H}, \mathrm{s}) .{ }^{19} \mathrm{~F}-\mathrm{NMR}\left(\mathrm{CDCl}_{3}\right) \delta:-25.3$ $(1 \mathrm{~F}, \mathrm{dd}, J=30,248 \mathrm{~Hz}),-26.8(1 \mathrm{~F}, \mathrm{dd}, J=16,248 \mathrm{~Hz}),-27.6-27.8(1 \mathrm{~F}$, $\mathrm{m}),-31.0-31.1(1 \mathrm{~F}, \mathrm{~m})$. MS $\mathrm{m} / \mathrm{z}(\%): 458\left(0.5, \mathrm{M}^{+}+1\right), 364(12$, $\mathrm{M}^{+}-\mathrm{SO}_{2} \mathrm{Et}$ ), $258(100)$. HRMS $\mathrm{m} / z$ : 364.1046 (Calcd for $\mathrm{C}_{18} \mathrm{H}_{14} \mathrm{~F}_{4} \mathrm{~N}_{3} \mathrm{O}$ : 364.1072). IR (KBr) cm $\mathrm{cm}^{-1}$ : 1050. Anal. Calcd for $\mathrm{C}_{20} \mathrm{H}_{19} \mathrm{~F}_{4} \mathrm{~N}_{3} \mathrm{O}_{3} \mathrm{~S}: \mathrm{C}, 52.51$; H, 4.19; N, 9.19. Found: C, 52.48; H, 4.22; N, 9.08.

Preparation of 1-[2-(2,4-Difluorophenyl)-3-methoxy-3-(methylsulfonyl)]-1H-1,2,4-triazole (11t) Diazomethane $\left(0.29 \mathrm{M} / \mathrm{l}\right.$ in $\left.\mathrm{Et}_{2} \mathrm{O} ; 10 \mathrm{ml}\right)$ was added to a solution of 2-(2,4-difluorophenyl)-2,2-difluoro-3-(methylsulfonyl)-1-(1H-1,2,4-triazol-1-yl)-2-propanol (2a) $(0.3 \mathrm{mmol})$ in $\mathrm{MeOH}$ $(10 \mathrm{ml})$ with stirring at $0^{\circ} \mathrm{C}$. The mixture was stirred at room temperature for $12 \mathrm{~h}$, and the solvent was evaporated under reduced pressure. The residue was chromatographed on a silica gel column using AcOEt- $n$-hexane $[1: 8$ $(\mathrm{v} / \mathrm{v})]$ to give 11t. Yield $82 \%$. Colorless oil. ${ }^{1} \mathrm{H}-\mathrm{NMR}\left(\mathrm{CDCl}_{3}\right) \delta: 3.03(3 \mathrm{H}$, s), $3.75(3 \mathrm{H}, \mathrm{s}), 5.18(1 \mathrm{H}, \mathrm{d}, J=15.6 \mathrm{~Hz}), 5.32(1 \mathrm{H}, \mathrm{d}, J=15.6 \mathrm{~Hz}), 6.79-$ $6.84(1 \mathrm{H}, \mathrm{m}), 6.85-6.91(1 \mathrm{H}, \mathrm{m}), 7.53-7.59(1 \mathrm{H}, \mathrm{m}), 7.78(1 \mathrm{H}, \mathrm{s}), 8.07$ $(1 \mathrm{H}, \mathrm{s}) .{ }^{19} \mathrm{~F}-\mathrm{NMR}\left(\mathrm{CDCl}_{3}\right) \delta:-25.4(1 \mathrm{~F}, \mathrm{dd}, J=30,248 \mathrm{~Hz}),-27.1(1 \mathrm{~F}, \mathrm{dd}$, $J=15,248 \mathrm{~Hz}),-27.9-28.2(1 \mathrm{~F}, \mathrm{~m}),-31.1-31.2(1 \mathrm{H}, \mathrm{m})$. FAB-MS $m / z: 368\left(\mathrm{M}^{+}+1\right)$. MS $m / z(\%) 368\left(0.6, \mathrm{M}^{+}+1\right), 288(100)$. HRMS $m / z$ : 368.0712 (Calcd for $\left.\mathrm{C}_{13} \mathrm{H}_{14} \mathrm{~F}_{4} \mathrm{~N}_{3} \mathrm{O}_{3} \mathrm{~S}: 368.0706\right)$. IR (KBr) cm ${ }^{-1}: 1400$.

Synthesis of 2-(2,4-Difluorophenyl)-3-(methylsulfonyl)-1-(1 H-1,2,4-triazol-1-yl)-2-propanol (12) 12 was prepared from $2^{\prime}, 4^{\prime}$-difluoro-2(methylthio)acetophenone. ${ }^{8)}$ Colorless powder. mp $149.5^{\circ} \mathrm{C} .{ }^{1} \mathrm{H}-\mathrm{NMR}$ $\left(\mathrm{CDCl}_{3}\right) \delta: 2.86(3 \mathrm{H}, \mathrm{s}), 3.42(1 \mathrm{H}, \mathrm{d}, J=15.1 \mathrm{~Hz}), 3.84(1 \mathrm{H}, \mathrm{d}, J=15.1 \mathrm{~Hz})$, $4.62(1 \mathrm{H}, \mathrm{d}, J=14.7 \mathrm{~Hz}), 4.88(1 \mathrm{H}, \mathrm{d}, J=14.7 \mathrm{~Hz}), 5.49(1 \mathrm{H}, \mathrm{s}), 6.83-6.92$ $(2 \mathrm{H}, \mathrm{m}), 7.50-7.57(1 \mathrm{H}, \mathrm{m}), 7.87(1 \mathrm{H}, \mathrm{s}), 8.04(1 \mathrm{H}, \mathrm{s}) .{ }^{19} \mathrm{~F}-\mathrm{NMR}\left(\mathrm{CDCl}_{3}\right)$ $\delta:-32.1-32.2(1 \mathrm{~F}, \mathrm{~m}),-33.6-33.8(1 \mathrm{~F}, \mathrm{~m}) . \mathrm{MS} m / z(\%): 318$ (3, $\left.\mathrm{M}^{+}+1\right), 121$ (100). HRMS m/z: 318.0704 (Calcd for $\mathrm{C}_{12} \mathrm{H}_{14} \mathrm{~F}_{2} \mathrm{~N}_{3} \mathrm{O}_{3} \mathrm{~S}$ : 318.0725). IR ( $\mathrm{KBr}) \mathrm{cm}^{-1}: 3100$. Anal. calcd. For $\mathrm{C}_{12} \mathrm{H}_{13} \mathrm{~F}_{2} \mathrm{~N}_{3} \mathrm{O}_{3} \mathrm{~S}: \mathrm{C}, 45.42$; H, 4.13; N, 13.24. Found: C, 45.63; H, 4.17; N, 13.48 .

In Vitro Antifungal Activities The MICs of the compounds were deter- mined by the National Committee for Clinical Laboratory Standards (NCCLS) guideline M27- $\mathrm{A}^{9}$ broth microdilution method for yeasts and by the NCCLS guideline M38- $\mathrm{P}^{10)}$ for filamentous fungi. The compounds were dissolved in DMSO (final concentration of DMSO: 1\%), and were tested at different concentrations (from 8 to $0.016 \mu \mathrm{g} / \mathrm{ml}$ ). C. albicans (ATCC 90028) and C. krusei (ATCC 6258) for yeast strains, and A. flavus (IFM 41935) and A. fumigatus (IFM 40808) for filamentous fungi strains were used. The MICs were measured after $48 \mathrm{~h}$ incubation at $35^{\circ} \mathrm{C}$.

In Vivo Antifungal Activities The therapeutic effect of the compounds of single or four-day repeated oral administration on a mouse model of systemic infection with C. albicans was investigated in comparison with that of FLCZ. Systemic candidosis was induced in four-week-old male ICR mice, and five or ten mice per group were used. Mice were infected intravenously by inoculation of $C$. albicans IFM 40009 suspension $\left(3 \times 10^{6}\right.$ cells/mouse) into the lateral tail vein. The compounds and FLCZ were dissolved in $20 \%$ polyethylene glycol 200 (doses were $5,1.25$ and $0.313 \mathrm{mg} / \mathrm{kg} / \mathrm{day}$ ), and were administered orally or i.v. $1 \mathrm{~h}$ after the challenge once or daily for 4 consecutive days. The therapeutic effect was presented as the duration of survival days until days 14 or 18 after infection, and were compared with control group. The significantly difference from control were obtained by KaplaneMiere method (cox-mantel test).

X-Ray Crystallographic Analysis of (-)-2a Diffraction measurments were performed on a RAXIS-RAPID diffractometer using the graphite monochromated $\mathrm{Cu}-K \alpha$ radiation $(\lambda=1.54178 \AA)$. The crystal data are as follows: $\mathrm{C}_{13} \mathrm{H}_{13} \mathrm{O}_{3} \mathrm{~N}_{3} \mathrm{~F}_{3} \mathrm{~S}$, F.W. $=367.32$, orthorhombic, space group $P 22_{1} 2_{1} 2_{1}$, $a=13.3423(5) \AA, b=20.1496(9) \AA, c=11.7259(4) \AA, V=3152.4(2) \AA^{3}, Z=8$. A total of 31618 reflections were measured, of which 5622 unique $\left(\mathrm{R}_{\text {int }}=0.066\right)$ reflections were used for analysis. The final $R$-factor and weighted $R$-factor were 0.042 and 0.040 , respectively. The structure was solved by the direct methods using SHELX86, ${ }^{11)}$ and expanded using Fourier techniques. ${ }^{12)}$ All calculations were performed using the teXsan crystallographic software package of Molecular Structure Corporation.

\section{References and Notes}

1) Eto H., Kaneko Y., Sakamoto T., Chem. Pharm. Bull., 48, 982-990 (2000).

2) Fromtling R. A., Drugs Future, 14, 1165-1168 (1989).

3) Oida S., Tajima Y., Konosu T., Nakamura Y., Somada A., Tanaka T., Habuki S., Harasaki T., Kamai Y., Fukuoka T., Ohya S., Yasuda H., Chem. Pharm. Bull., 48, 694-707 (2000).

4) Matsumoto M., Asaoka T., Iwasa A., Ikada Y., Yamamoto K., Hirayama F., Abstracts of Papers, The 36th Interscience Conference on Antimicrobial Agents and Chemotherapy, New Orleans, Sep. 1996, p. 113.

5) Takeda S., Kaneko Y., Eto H., Tokizawa M., Sato S., Yoshida K., Namiki S., Ogawa M., Chem. Pharm. Bull., 48, 1097-1100 (2000).

6) Richardson K., Whittle, P. J., Eur. Patent EP-117100 [Chem. Abstr., 102, 24632 (1984)].

7) Tokizawa M., Takeda S., Kaneko Y., Eto H., Ishida K., Maebashi K., Matsumoto M., Asaoka T., Sato S., Matsuda H., Japan Kokai Tokko Koho, JP 07-335251, Dec. 22, 1995 [Chem. Abstr., 127, 121738 (1995)].

8) Gymer G. E., Richardson K., Eur. Patent EP-95828 (1983) [Chem. Abstr., 100, 85706 (1983)].

9) National Committee for Clinical Laboratory Standards. 1997. Reference method for broth dilution antifungal susceptibility testing for yeasts. Approval standard. Document M27-A. National Committee for Clinical Laboratory Standards, Wayne, Pa.

10) National Committee for Clinical Laboratory Standards. 1998. Reference method for broth dilution antifungal susceptibility testing for conidium-forming filamentous fungi. Proposed standard. Document M38-P. National Committee for Clinical Laboratory Standards, Wayne, Pa.

11) Sheldrick G. M., "Crystallographic Computing 3," eds. by Sheldrick G. M., Kruger C., Goddard R., Oxford University Press, 1985, pp. $175-189$.

12) Beurskens P. T., Admiraal G., Beurskens G., Bosman W. P., de Gelder R., Israel R., Smits J. M. M., The DIRDIF-94 program system, Technical Report of the Crystallography Laboratory, University of Nijmegen, The Netherlands (1994). 